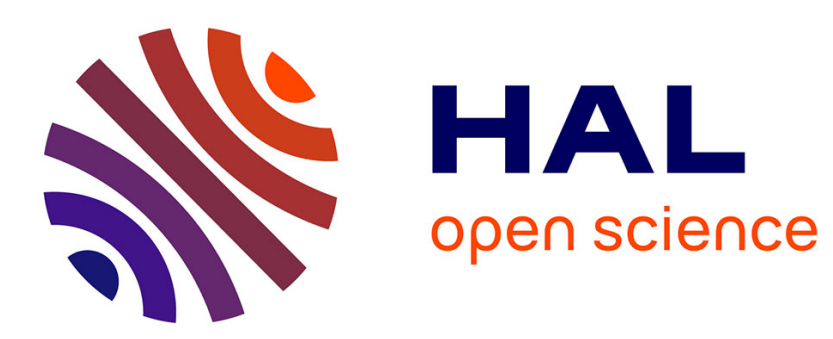

\title{
Effective Algorithms for Parametrizing Linear Control Systems over Ore Algebras
}

Frédéric Chyzak, Alban Quadrat, Daniel Robertz

\section{To cite this version:}

Frédéric Chyzak, Alban Quadrat, Daniel Robertz. Effective Algorithms for Parametrizing Linear

Control Systems over Ore Algebras. [Research Report] RR-5181, INRIA. 2004. inria-00071407

\section{HAL Id: inria-00071407 \\ https://hal.inria.fr/inria-00071407}

Submitted on 23 May 2006

HAL is a multi-disciplinary open access archive for the deposit and dissemination of scientific research documents, whether they are published or not. The documents may come from teaching and research institutions in France or abroad, or from public or private research centers.
L'archive ouverte pluridisciplinaire HAL, est destinée au dépôt et à la diffusion de documents scientifiques de niveau recherche, publiés ou non, émanant des établissements d'enseignement et de recherche français ou étrangers, des laboratoires publics ou privés. 
INSTITUT NATIONAL DE RECHERCHE EN INFORMATIQUE ET EN AUTOMATIQUE

\section{Effective Algorithms for Parametrizing Linear Control Systems over Ore Algebras}

Frédéric Chyzak, Alban Quadrat, Daniel Robertz

$\mathbf{N}^{\circ} 5181$

$29 / 04 / 04$

Thème SYM 



\title{
Effective Algorithms for Parametrizing Linear Control Systems over Ore Algebras
}

\author{
Frédéric Chyzak*, Alban Quadrat ${ }^{\dagger}$, Daniel Robertz ${ }^{\ddagger}$ \\ Thème SYM - Systèmes symboliques \\ Projets ALGO \& CAFÉ
}

Rapport de recherche $n^{\circ} 5181-29 / 04 / 04-44$ pages

\begin{abstract}
In this paper, we study linear control systems over Ore algebras. Within this mathematical framework, we can simultaneously deal with different classes of linear control systems such as time-varying systems of ordinary differential equations (ODEs), differential time-delay systems, underdetermined systems of partial differential equations (PDEs), multidimensional discrete systems, multidimensional convolutional codes etc. We give effective algorithms which check whether or not a linear control system over some Ore algebra is controllable, parametrizable, flat or $\pi$-free.
\end{abstract}

Key-words: Linear systems over Ore algebras, controllability, parametrization, flatness, $\pi$-freeness, effective algorithms, non-commutative Gröbner bases.

* INRIA Rocquencourt, ALGO project, INRIA Rocquencourt, Domaine de Voluceau BP 105, 78153 Le Chesnay cedex, France. frederic.chyzak@inria.fr.

$\dagger$ INRIA Sophia Antipolis, CAFE project, 2004, Route des Lucioles BP 93, 06902 Sophia Antipolis cedex, France. Alban.Quadrat@sophia.inria.fr.

$\ddagger$ Lehrstuhl B für Mathematik RWTH - Aachen, Templergraben 64, 52056 Aachen, Germany. daniel@momo.math.rwth-aachen.de. The third author has been financially supported by the CTS grant HPMTCT-2001-00278 during his stays at INRIA Sophia Antipolis. 


\section{Algorithmes effectifs pour la paramétrisation des systèmes de contrôle linéaires sur des algèbres de Ore}

Résumé : Dans ce papier, nous étudions les systèmes de contrôle linéaires sur des algèbres de Ore. Cette approche nous permet d'étudier dans un même cadre mathématique différentes classes de systèmes de contrôle linéaires telles que les systèmes d'équations différentielles ordinaires (EDOs) variants dans le temps, les systèmes différentiels à retards, les systèmes sous-déterminés d'équations aux dérivées partielles (EDPs), les systèmes multidimensionnels discrets, les codes convolutionnels multidimensionnels etc. Nous donnons des algorithmes effectifs vérifiant si un système de contrôle linéaire sur une algèbre de Ore est contrôlable, paramétrisable, plat ou $\pi$-libre.

Mots-clés : Systèmes linéaires sur des algèbres de Ore, contrôlabilité, paramétrisation, platitude, $\pi$-liberté, algorithmes effectifs, bases de Gröbner non-commutatives. 


\section{Introduction}

Over the last thirty years, for practical and theoretical reasons, different new classes of linear control systems have been introduced such as differential time-delay systems, multidimensional systems, partial differential equations, convolutional codes, hybrid systems... All these classes of systems are governed by new types of mathematical equations and have needed new techniques in order to analyze their structural properties and to synthesize new control laws. This growth of new types of control systems has led to generalize some previously known results and techniques so that they could be used for more general classes of systems. The main interest is to get similar concepts, techniques and algorithms for studying different classes of linear systems.

In this paper, we study linear control systems over Ore algebras. An Ore algebra is an algebra of non-commutative functional operators which satisfy certain commutation rules. For instance, differential/time-delay/advance/discrete shift/divided difference... operators are examples of elements of some Ore algebras. Within this mathematical framework, we can simultaneously deal with different classes of linear control systems such as time-varying ordinary differential systems (ODEs), differential time-delay systems, partial differential equations (PDEs), multidimensional discrete systems... Moreover, the recent extension of Gröbner bases to some non-commutative polynomial rings allows us to work effectively in Ore algebras $[6,7]$.

The purpose of this paper is to give effective algorithms which check whether or not a linear control system over some Ore algebra is controllable, parametrizable, flat or $\pi$-free. These problems have been intensively studied in $[11,20,21]$ for linear differential time-delay systems and, in [22, 24, $25,26,27,29,30,31,32,36,37]$, for linear multidimensional systems. The main novelty of this paper is to present algorithms which work for both classes of systems as well as for new ones. In particular, our approach allows us to effectively obtain parametrizations of a controllable plant and flat outputs of a flat system. Let us notice that such algorithms were still missing for linear differential time-delay systems (see $[11,20,21]$ for more details). The results developed in this paper give new effective machinery for the study of the structural properties of linear systems. We hope that they could play important roles for the study of motion planning or tracking [11, 20, 21].

Ore algebras are rings of non-commutative polynomials that represent linear functional operators in a natural way. A recent extension of the theory of Gröbner bases to this setting [5] makes manipulations of (one-sided) ideals of Ore algebras effective. We recall the basics of this theory in Section 2. As is the case for linear differential systems of algebraic analysis, a fundamental remark is that a module over an Ore algebra is canonically associated with any linear control system. Before we provide the readers in Section 4 with a dictionary, first introduced in [30], between structural properties of control systems and algebraic properties of modules, we recall in Section 3 the definitions and results of module theory to be used in the rest of the article. Next, the question is to algorithmically decide the algebraic properties of modules. This is done by means of homological algebra in Sections 5 and 7. The good point is that the central homological objects as syzygy modules, free resolutions, and extension modules can all be computed by algorithms which we recall and exemplify on cases of application. In passing, the classical notion of involution, which exchanges left and right module structures, is recalled in Section 6, where explicit examples of involutions of Ore algebras that appear in control theory are also given.

Beside realizing the applicability of non-commutative Gröbner bases to make the theory algorithmic, the main contribution of the paper lies in the new approach for the proofs of algorithms in Sections 8 and 9 . These sections extend earlier results, previously obtained in special situations only, to the broad setting of linear control systems over Ore algebras. After collecting further results of homological algebra that could prove difficult to find in the literature, Section 8 gives an algorithm to determine whether a control system is parametrizable and, if so, to compute a parametrization, while, if not, to compute a basis of autonomous elements. Section 9 restricts to control systems over commutative Ore algebras in order to provide algorithms for deciding flatness, for studying $\pi$-freeness, and when they exist, for computing left and right inverses and minimal parametrizations.

In an appendix, we have illustrated all the main results and algorithms of the paper with explicit examples. All the computations were done using the Maple package OreModules written by the authors based on the library Mgfun [6]. These libraries as well as all the example worksheets of the appendix are available on the web page http://wwwb.math.rwth-aachen.de/OreModules. Some results are new (e.g., the parametrization of the electric transmission line [20,34]) and we believe that these examples have some educational interest.

Some of the results developed in this paper were firstly presented in the conference papers $[8,9]$. 


\section{Ore algebras}

\subsection{Definition and examples}

In order to deal with different classes of linear systems (ODEs, PDEs, differential time-delay systems, discrete systems, multidimensional systems...) in a unified framework, we represent them by means of matrices with entries in an Ore algebra of functional operators.

Definition 2.1. 1. [17] Let $A$ be an integral domain (i.e., $a b=0, a \neq 0 \Rightarrow b=0$ ). The skew polynomial ring $A[\partial ; \sigma, \delta]$ is the non-commutative ring consisting of all polynomials in $\partial$ with coefficients in $A$ obeying the commutation rule

$$
\partial a=\sigma(a) \partial+\delta(a), \quad a \in A,
$$

where $\sigma$ is a $k$-algebra endomorphism of $A$, namely $\sigma: A \rightarrow A$ satisfies

$$
\left\{\begin{array}{l}
\sigma(1)=1, \\
\sigma(a+b)=\sigma(a)+\sigma(b), \quad a, b \in A, \\
\sigma(a b)=\sigma(a) \sigma(b), \quad a, b \in A,
\end{array}\right.
$$

and $\delta$ is a $\sigma$-derivation of $A$, namely $\delta: A \rightarrow A$ satisfies:

$$
\left\{\begin{array}{l}
\delta(a+b)=\delta(a)+\delta(b), \quad a, b \in A \\
\delta(a b)=\sigma(a) \delta(b)+\delta(a) b, \quad a, b \in A .
\end{array}\right.
$$

2. [7] Let $A=k\left[x_{1}, \ldots, x_{n}\right]$ be a commutative polynomial ring over a field $k$ (if $n=0$ then $A=k$ ). The iterated skew polynomial ring $D=A\left[\partial_{1} ; \sigma_{1}, \delta_{1}\right] \ldots\left[\partial_{m} ; \sigma_{m}, \delta_{m}\right]$ is called Ore algebra if the $\sigma_{i}$ 's and $\delta_{j}$ 's commute for $1 \leq i, j \leq m$ and satisfy:

$$
\sigma_{i}\left(\partial_{j}\right)=\partial_{j}, \quad \delta_{i}\left(\partial_{j}\right)=0, \quad j<i .
$$

Remark 2.2. $[7,17]$ Let $D=A[\partial ; \sigma, \delta]$ be a skew polynomial ring. Every element $P$ of $D$ has a unique normal form which is given by $P=\sum_{i=1}^{n} a_{i} \partial^{i}$ for suitable $a_{i} \in A$ and $n \in \mathbb{Z}_{\geq 0}$. If $a_{n} \neq 0$, then the degree of $P$ is $n$. For every Ore algebra, we get a similar normal form of its elements by moving all products of $\partial_{1}, \ldots, \partial_{m}$ on the right in each summand.

Example 2.3. Our first example is the Weyl algebra $A_{1}=k[t][\partial ; \sigma, \delta]$, where:

$$
\sigma=\operatorname{id}_{k[t]}, \quad \delta=\frac{d}{d t}
$$

Rule (1) expresses the commutation of the operator which acts as differentiation w.r.t. $t$ :

$$
\partial a=a \partial+\frac{d a}{d t}, \quad a \in k[t], \quad \text { to compare with } \quad \partial(a y)=a \partial y+\left(\frac{d a}{d t}\right) y .
$$

For instance, we associate $R=[\partial I-A(t):-B(t)] \in A_{1}^{n \times(n+m)}$ with the time-varying Kalman system $\dot{x}(t)=A(t) x(t)+B(t) u(t)$, where $A$ and $B$ are polynomial matrices in $t$.

Similar to polynomial rings in $2 n$ indeterminates, we can define the so-called Weyl algebra $A_{n}=$ $k\left[x_{1}, \ldots, x_{n}\right]\left[\partial_{1} ; \sigma_{1}, \delta_{1}\right] \ldots\left[\partial_{n} ; \sigma_{n}, \delta_{n}\right]$, where $\sigma_{i}$ and $\delta_{i}$ on $k\left[x_{1}, \ldots, x_{n}\right]$ are the maps

$$
\sigma_{i}=\mathrm{id}_{k\left[x_{1}, \ldots, x_{n}\right]}, \quad \delta_{i}=\frac{\partial}{\partial x_{i}}, \quad i=1, \ldots, n,
$$

and every other commutation rule is prescribed by Definition 2.1. In particular, we have:

$$
\partial_{i} x_{j}=x_{j} \partial_{i}+\delta_{i j}, \quad 1 \leq i, j \leq n, \quad \text { where } \delta_{i j}=1 \text {, if } i=j, \text { and } 0 \text { else. }
$$

Example 2.4. The algebra of shift operators with polynomial coefficients is another special case of an Ore algebra. For $h \in \mathbb{R}$, we define $S_{h}=k[t]\left[\delta_{h} ; \sigma_{h}, \delta\right]$ by:

$$
\sigma_{h}(a)(t)=a(t-h), \quad \delta(a)=0, \quad a \in k[t] .
$$

Hence, the commutation rule $\delta_{h} t=(t-h) \delta_{h}$ actually represents the action of the shift operator on polynomials. For instance, the matrix of operators $R=\left[1-\delta_{h}:-\delta_{h}^{2}\right] \in S_{h}^{1 \times 2}$ is associated with the time-delay system $x(t)=x(t-h)+u(t-2 h)$. Let us notice that $\partial_{h}$ is a time-delay operator if $h>0$ and an advance operator if $h<0$. 
Example 2.5. In order to treat differential time-delay systems, we mix the constructions of the two preceding examples. We define the Ore algebra $D_{h}=k[t]\left[\partial ; \sigma_{1}, \delta_{1}\right]\left[\delta_{h} ; \sigma_{2}, \delta_{2}\right]$, where:

$$
\sigma_{1}=\mathrm{id}_{k[t]}, \quad \delta_{1}=\frac{d}{d t}, \quad \sigma_{2}(a)(t)=a(t-h), \quad \delta_{2}=0, \quad h \in \mathbb{R}, \quad a \in k[t] .
$$

For instance, we associate the matrix $R=\left[\partial I-A(t):-B(t) \delta_{h}\right] \in D_{h}^{n \times(n+m)}$ with the system $\dot{x}(t)=A(t) x(t)+B(t) u(t-h)$, where $A$ and $B$ are polynomial matrices in $t$.

If the considered system also involves the advance operator, which is the inverse operator of the time-delay operator, then we may work with the Ore algebra

$$
H_{h}=k[t]\left[\partial ; \sigma_{1}, \delta_{1}\right]\left[\delta_{h} ; \sigma_{2}, \delta_{2}\right]\left[\tau_{h} ; \sigma_{3}, \delta_{3}\right],
$$

where $\sigma_{i}, \delta_{i}, i=1,2$, are as above and:

$$
\sigma_{3}(a)(t)=a(t+h), \quad \delta_{3}=0, \quad a \in k[t] .
$$

We then compute with elements of $H_{h}$ modulo $\delta_{h} \tau_{h}=\tau_{h} \delta_{h}=1$.

Example 2.6. In order to study multidimensional discrete linear systems, we define the following Ore algebra $D=k\left[x_{1}, \ldots, x_{n}\right]\left[\partial_{1} ; \sigma_{1}, \delta_{1}\right] \ldots\left[\partial_{n} ; \sigma_{n}, \delta_{n}\right]$, where $\sigma_{i}$ and $\delta_{i}$ on $k\left[x_{1}, \ldots, x_{n}\right]$ are the maps:

$$
\sigma_{i}(a)\left(x_{1}, \ldots, x_{n}\right)=a\left(x_{1}, \ldots, x_{i-1}, x_{i}+1, x_{i+1}, \ldots, x_{n}\right), \quad \delta_{i}=0, \quad i=1, \ldots, n .
$$

Similarly to Example 2.5, we can define an Ore algebra which combines the shift operator $\sigma_{i}$ and the inverse shift $\tau_{i}$ defined by $\tau_{i}(a)\left(x_{1}, \ldots, x_{n}\right)=a\left(x_{1}, \ldots, x_{i-1}, x_{i}-1, x_{i+1}, \ldots, x_{n}\right)$. Such a construction is then a generalization of the Laurent polynomial ring to non-commutative polynomials.

Ore algebras based on other functional operators can also be defined (discrete shifts, divided differences, $q$-shift, Eulerian operators...). We refer to $[7,17]$ for more examples.

\subsection{Properties of Ore algebras \& Gröbner bases}

We summarize the most important properties of Ore algebras that will enable us to computationally deal with modules over Ore algebras.

Proposition 2.7. [7] If $A$ has the left Ore property (resp. right Ore property), namely, for each pair $\left(a_{1}, a_{2}\right) \in A \times A$, there is a pair $(0,0) \neq\left(b_{1}, b_{2}\right) \in A \times A$ such that $b_{1} a_{1}=b_{2} a_{2}$ (resp. $\left.a_{1} b_{1}=a_{2} b_{2}\right)$, then $A[\partial ; \sigma, \delta]$ has also the left (resp. right) Ore property.

Proposition 2.8. [17] If $A$ is an integral domain and $\sigma$ is injective, then the skew polynomial ring $A[\partial ; \sigma, \delta]$ is an integral domain.

Proposition 2.9. [17] If $A$ is a left noetherian ring and $\sigma$ is an automorphism (e.g., $A_{n}, S_{h}, D_{h}$, $\left.H_{h}\right)$, then the skew polynomial ring $A[\partial ; \sigma, \delta]$ is a left noetherian ring.

In order to study systems over (non-commutative) polynomial rings effectively, we need to introduce some algorithmic methods based on Gröbner bases. We first need monomial orders to compare (noncommutative) polynomials.

Definition 2.10. $\quad$ 1. Let $D$ be an Ore algebra. A monomial order $<$ on $D$ is defined as a total order on the set of monomials $\operatorname{Mon}(D)$ satisfying $1<m$ for all monomials $m \in \operatorname{Mon}(D)$ and, if $m_{1}<m_{2}$ holds for two monomials $m_{1}, m_{2} \in \operatorname{Mon}(D)$, then $m_{1} \cdot n<m_{2} \cdot n$ for all $n \in \operatorname{Mon}(D)$.

2. Given a polynomial $P \in D$ and a monomial order $<$, we can compare the monomials with a non-zero coefficient in $P$ w.r.t. $<$. The greatest of these monomials is the leading monomial $\operatorname{lm}(P)$ of $P$.

Definition 2.11. [1] Let $A$ be a polynomial ring and $I$ an ideal of $A$. A set of non-zero polynomials $G=\left\{g_{1}, \ldots, g_{t}\right\}$ is called a Gröbner basis for $I$ if for all $0 \neq f \in I$, there exists $1 \leq i \leq t$ such that $\operatorname{lm}\left(g_{i}\right)$ divides $\operatorname{lm}(f)$.

Remark 2.12. A consequence of the condition that defines Gröbner bases is that every polynomial $f$ in $I$ is reduced to 0 modulo $G$, i.e., by iterative division of $f$ by suitable $g_{i} \in G$ we obtain the zero polynomial. 
For the case of commutative polynomial rings, Buchberger's algorithm $([1,2])$ computes Gröbner bases of polynomial ideals. The next theorem states that this algorithm can be extended to certain Ore algebras. Every Ore algebra within our scope is of this kind.

Theorem 2.13. [7, 12] Let $A=k\left[x_{1}, \ldots, x_{n}\right]$ be the polynomial ring with $n$ indeterminates over the field $k$ and $A\left[\partial_{1} ; \sigma_{1}, \delta_{1}\right] \ldots\left[\partial_{m} ; \sigma_{m}, \delta_{m}\right]$ an Ore algebra with

$$
\sigma_{i}\left(x_{j}\right)=a_{i j} x_{j}+b_{i j}, \quad \delta_{i}\left(x_{j}\right)=c_{i j} \quad 1 \leq i \leq m, \quad 1 \leq j \leq n,
$$

for certain $a_{i j} \in k \backslash\{0\}, b_{i j} \in k, c_{i j} \in A$. If the $c_{i j}$ are of total degree at most 1 in the $x_{i}$ 's, then a non-commutative version of Buchberger's algorithm terminates for any monomial order on $x_{1}, \ldots, x_{n}$, $\partial_{1}, \ldots, \partial_{m}$, and its result is a Gröbner basis w.r.t. the given monomial order.

An important technique that uses Gröbner bases is elimination of variables.

Definition 2.14. [1] Let $D$ be the polynomial ring over $k$ with variables $x_{1}, \ldots, x_{n}, y_{1}, \ldots, y_{m}$. Assume that monomial orders $<_{x}$ and $<_{y}$ on the monomials that contain only the $x_{i}$ 's or $y_{i}$ 's resp. are given. An elimination order is then defined by

$$
m_{1} \cdot n_{1}<m_{2} \cdot n_{2} \Longleftrightarrow m_{1}<{ }_{x} m_{2} \quad \text { or } \quad m_{1}=m_{2} \text { and } n_{1}<_{y} n_{2},
$$

where $m_{1}, m_{2}$ (resp. $n_{1}, n_{2}$ ) are monomials containing only the $x_{i}$ 's (resp. $y_{i}$ 's).

The elimination order which we shall use in this paper is the one induced by the degree reverse lexicographical orders on $x_{1}, \ldots, x_{n}$ and $y_{1}, \ldots, y_{m}$ resp.. This is a very common order called lexdeg in the Maple package Groebner.

Example 2.15. Given an ideal $I$ of $D$, we obtain a Gröbner basis of the ideal $I \cap k\left[y_{1}, \ldots, y_{m}\right]$ by computing the Gröbner basis $G$ of $I$ w.r.t. an elimination order and intersecting $G$ with $k\left[y_{1}, \ldots, y_{m}\right]$ (which amounts only to omit all polynomials in $G$ that involve any $x_{i}$ ).

\section{Module theory over Ore algebras}

Let us give a motivation for the use of modules. Let $D$ be an integral domain and let us consider a system of equations

$$
\sum_{j=1}^{p} R_{i j} y_{j}=0, \quad 1 \leq i \leq q,
$$

where $R_{i j} \in D, p, q \in \mathbb{N}$. By collecting the coefficients $R_{i j}$, we obtain a matrix $R \in D^{q \times p}$ which, multiplied by the column vector $y=\left(y_{1}: \ldots: y_{p}\right)^{T}$, yields system (3) again.

Let us consider the following left $D$-morphism ( $D$-linear map):

$$
\begin{aligned}
& D^{1 \times q} \stackrel{\cdot R}{\longrightarrow} D^{1 \times p}, \\
&\left(P_{1}: \ldots: P_{q}\right) \longmapsto \\
&
\end{aligned}
$$

Then, im $R=D^{1 \times q} R$ is the left $D$-module generated by the left $D$-linear combinations of the rows of $R$ (namely, the ring $D$ acts on the elements of im .R= $D^{1 \times q} R$ from the left).

Let us show how system (3) corresponds to the left $D$-module $M=D^{1 \times p} / D^{1 \times q} R$. We denote by $\left\{e_{i}\right\}_{1 \leq i \leq p}$ (resp. $\left\{f_{j}\right\}_{1 \leq j \leq q}$ ) the canonical basis of $D^{1 \times p}$ (resp. $D^{1 \times q}$ ), namely $e_{i}$ is the row vector with

1 in $i$ th position and 0 elsewhere. Let us define by $\pi: D^{1 \times p} \rightarrow M$ the left $D$-morphism which maps every element of $D^{1 \times p}$ to its residue class in $M$, i.e., modulo $D^{1 \times q} R\left(\pi\left(\lambda_{1}\right)=\pi\left(\lambda_{2}\right) \Leftrightarrow \exists \mu \in D^{1 \times q}\right.$ such that $\left.\lambda_{1}-\lambda_{2}=\mu R\right)$. Then, for $i=1, \ldots, q$, we have

$$
f_{j} R=\left(R_{j 1}: \ldots: R_{j p}\right)=\sum_{i=1}^{p} R_{j i} e_{i} \in D^{1 \times q} R \Rightarrow \pi\left(f_{j} R\right)=\pi\left(\sum_{i=1}^{p} R_{j i} e_{i}\right)=\sum_{i=1}^{p} R_{j i} \pi\left(e_{i}\right)=0,
$$

and thus, if we denote by $y_{i}=\pi\left(e_{i}\right)$ the residue class of $e_{i}$ in $M$, then $M$ is defined by

$$
\sum_{i=1}^{p} R_{j i} y_{i}=0, \quad 1 \leq j \leq q, \quad \Leftrightarrow \quad R y=0
$$

as well as by the left $D$-linear combinations of its equations, where $y=\left(y_{1}: \ldots: y_{p}\right)^{T}$. The left $D$-module $M=D^{1 \times p} / D^{1 \times q} R$ is finitely generated because every element $m \in M$ can be written as $m=\sum_{i=1}^{p} P_{i} y_{i}$, where $P_{i} \in D$. 
Definition 3.1. We say that the finitely generated left $D$-module $M=D^{1 \times p} / D^{1 \times q} R$ is associated with (or corresponds to) (3).

Let us recall that if $D$ is a commutative ring, then a left $D$-module $M$ is also a right $D$-module and conversely. In this case, we shall only say that $M$ is a $D$-module.

Example 3.2. Let us consider an Ore algebra $D_{h}=\mathbb{R}(a, k, \zeta, \omega)\left[\partial ; \sigma_{1}, \delta_{1}\right]\left[\delta_{h} ; \sigma_{2}, \delta_{2}\right]$ of the type of Example 2.5 and revisit the following wind tunnel model defined in [19]

$$
\left\{\begin{array}{l}
\dot{x}_{1}(t)=-a x_{1}(t)+k a x_{2}(t-h), \\
\dot{x}_{2}(t)=x_{3}(t) \\
\dot{x}_{3}(t)=-\omega^{2} x_{2}(t)-2 \zeta \omega x_{3}(t)+\omega^{2} u(t),
\end{array}\right.
$$

where $a, k, \zeta$ and $\omega$ are real constants. System (4) gives rise to the following matrix

$$
R=\left(\begin{array}{cccc}
\partial+a & -k a \delta_{h} & 0 & 0 \\
0 & \partial & -1 & 0 \\
0 & \omega^{2} & \partial+2 \zeta \omega & -\omega^{2}
\end{array}\right) \in D_{h}^{3 \times 4},
$$

and thus, system (4) corresponds to the (left) $D_{h}$-module $M=D_{h}^{1 \times 4} / D_{h}^{1 \times 3} R$.

In Section 4, we shall develop a dictionary between the properties of the left $D$-module $M$ and the properties of the system $R y=0$. But, at first, let us introduce some defintions of module theory and homological algebra.

Definition 3.3. [33] Let $D$ be an integral domain.

1. A family $\left(M_{i}\right)_{i \in \mathbb{Z}}$ of $D$-modules together with a family $\left(d_{i}\right)_{i \in \mathbb{Z}}$ of $D$-module morphisms $d_{i}$ : $M_{i} \rightarrow M_{i-1}$ is a complex, if $d_{i} \circ d_{i+1}=0$ for all $i \in \mathbb{Z}$. We write:

$$
\ldots \stackrel{d_{i+2}}{\longrightarrow} M_{i+1} \stackrel{d_{i+1}}{\longrightarrow} M_{i} \stackrel{d_{i}}{\longrightarrow} M_{i-1} \stackrel{d_{i-1}}{\longrightarrow} \ldots
$$

The complex (6) is called exact at position $i$ if the defect of exactness of (6) at position $i$,

$$
H\left(M_{i}\right)=\operatorname{ker} d_{i} / \operatorname{im} d_{i+1},
$$

is equal to 0 or, equivalently, if $\operatorname{ker} d_{i}=\operatorname{im} d_{i+1}$. The complex (6) is called exact if it is exact at every position.

2. The exact sequence $0 \longrightarrow M^{\prime} \stackrel{f}{\longrightarrow} M \stackrel{g}{\longrightarrow} M^{\prime \prime} \longrightarrow 0$, i.e., where $f$ is injective, $g$ is surjective and $\operatorname{ker} g=\operatorname{im} f$, is called a short exact sequence.

We recall some properties of $D$-modules that will be important in the course of the paper.

Definition 3.4. [33] Let $D$ be an integral domain which is a left Ore ring and $M$ a finitely generated left $D$-module.

1. The left $D$-module $M$ is free if it is isomorphic to $D^{1 \times s}$ for a certain $s \in \mathbb{Z}_{\geq 0}$.

2. $M$ is a stably free left $D$-module if there exist $r, s \in \mathbb{Z}_{\geq 0}$ such that $M \oplus D^{1 \times r} \cong D^{1 \times s}$.

3. $M$ is a projective left $D$-module if there exist a left $D$-module $N$ and $s \in \mathbb{Z}_{\geq 0}$ such that $M \oplus N \cong D^{1 \times s}$.

4. Define by $\operatorname{hom}_{D}(M, D)$ the right $D$-module consisting of all $D$-morphisms $M \rightarrow D$. The set $\operatorname{hom}_{D}\left(\operatorname{hom}_{D}(M, D), D\right)$ is again a left $D$-module and there is a canonical map

$$
\varepsilon_{M}: M \longrightarrow \operatorname{hom}_{D}\left(\operatorname{hom}_{D}(M, D), D\right), \quad \varepsilon_{M}(m)(f)=f(m),
$$

$m \in M, f \in \operatorname{hom}_{D}(M, D)$. If $\varepsilon_{M}$ is an isomorphism, then $M$ is called reflexive left $D$-module.

5. The set $t(M)=\{m \in M \mid \exists 0 \neq P \in D: P m=0\}$ is a left submodule of $M$ which is called the torsion submodule of $M$. The non-zero elements of $t(M)$ are all the torsion elements of $M$. 
6. The left $D$-module $M$ is called torsion-free if its torsion submodule is trivial, i.e., $t(M)=0$.

We have similar definitions for right $D$-modules over integral domains which are right Ore rings.

Let us notice that the fact that $t(M)$ is a (left/right) $D$-submodule of $M$ implies that we have the short exact sequence:

$$
0 \longrightarrow t(M) \longrightarrow M \longrightarrow M / t(M) \longrightarrow 0 .
$$

Proposition 3.5. [33] Let $D$ be an integral domain which is a (left/right) Ore ring and $M$ be a finitely generated (left/right) D-module. Then, we have the following implications among these concepts:

$$
\text { free } \Rightarrow \text { projective } \Rightarrow \text { reflexive } \Rightarrow \text { torsion-free. }
$$

Theorem 3.6. 1. $\quad$ 17, 33] If $D$ is a left hereditary ring - namely, every left ideal of $D$ is a left projective D-module - (e.g., a commutative Dedekind domain, $\left.D=A_{1}\right)$, then every finitely generated torsion-free left $D$-module is projective. Moreover, if $D$ is a left principal ideal domain - namely, every left ideal of $D$ is principal - (e.g., $k[x]$ or $k(t)\left[\partial ; \operatorname{id}_{k(t)}, \frac{d}{d t}\right]$, where $k$ is a field of constants), then every finitely generated torsion-free left $D$-module is free.

2. [17, 33] Every projective module over a commutative polynomial ring with coefficients in a field is free (Quillen-Suslin theorem).

Definition 3.7. [33] A short exact sequence of (left/right) $D$-modules $0 \longrightarrow M^{\prime} \stackrel{f}{\longrightarrow} M \stackrel{g}{\longrightarrow} M^{\prime \prime} \longrightarrow 0$ is called a split exact sequence if one of the following equivalent conditions is satisfied:

- there exists an $D$-morphism $h: M^{\prime \prime} \longrightarrow M$ such that $g \circ h=i d_{M^{\prime \prime}}$,

- there exists an $D$-morphism $k: M \longrightarrow M^{\prime}$ such that $k \circ f=i d_{M^{\prime}}$,

- the (left/right) $D$-module $M$ is isomorphic to the direct sum of $M^{\prime}$ and $M^{\prime \prime}$, which is denoted by $M \cong M^{\prime} \oplus M^{\prime \prime}$, or equivalently, there exist two $D$-morphisms

$$
\left\{\begin{array}{l}
\phi=\left(\begin{array}{c}
k \\
g
\end{array}\right): M \longrightarrow M^{\prime} \oplus M^{\prime \prime}, \\
\psi=(f: h): M^{\prime} \oplus M^{\prime \prime} \longrightarrow M,
\end{array}\right.
$$

satisfying $\phi \circ \psi=i d_{M^{\prime} \oplus M^{\prime \prime}}$ and $\psi \circ \phi=i d_{M}$.

Proposition 3.8. [4, 33] If $M^{\prime \prime}$ is a (left/right) projective D-module and $M^{\prime}$ and $M$ are (left/right) $D$-modules, then the short exact sequence $0 \longrightarrow M^{\prime} \stackrel{f}{\longrightarrow} M \stackrel{g}{\longrightarrow} M^{\prime \prime} \longrightarrow 0$ splits.

In the course of the paper, we shall need the following classical lemma.

Lemma 3.9. [33] Let $D$ be a commutative noetherian ring, $M$ a finitely generated $D$-module and $S$ a multiplicatively closed subset of $D$ (namely, $1 \in S$ and $\forall s_{1}, s_{2} \in S \Rightarrow s_{1} s_{2} \in S$ ). Let us introduce the commutative noetherian ring $S^{-1} D=\{a / s \mid a \in D, s \in S\}$ and the $S^{-1} D$-module $S^{-1} D \otimes_{D} M=\{m / s \mid m \in M, s \in S\}$. Then, we have:

$$
S^{-1} D \otimes_{D} \operatorname{ext}_{D}^{j}(M, D) \cong \operatorname{ext}_{S^{-1}}^{j}\left(S^{-1} D \otimes_{D} M, S^{-1} D\right), \quad j \geq 0 .
$$

In the following sections, we shall develop effective algorithms based on Gröbner bases in order to check whether or not a left $D$-module $M$ associated with a linear control system (e.g., the differential time-delay system (4)) is torsion-free, reflexive, ..., or free. In the next section, we shall give some system interpretations of these properties of modules.

\section{System interpretations of module properties}

In what follows, we shall not precise the Ore algebra $D$ we use (e.g., if we study time-varying ordinary differential systems (resp. differential time-delay systems...), then $D=A_{1}\left(\right.$ resp. $\left.D=D_{h} \ldots\right)$ ).

Let us give some system interpretations of the previous properties of modules by introducing some definitions which generalize to linear systems over Ore algebras some definitions commonly used in the literature for some particular classes of systems (e.g., ordinary/partial differential equations, differential time-delay systems). In the following definition and the next proposition, the references therein refer to the particular classes of systems for which these concepts have been firstly introduced. 
Definition 4.1. - $[25,36]$ An observable of a linear control system $R y=0$ is a scalar $D$-linear combination of the components of $y$ (i.e., of the system variables including inputs, states, outputs). An observable $\phi(y)$ is called autonomous if it satisfies some linear equation by itself, namely $P \phi(y)=0$, where $0 \neq P \in D$. An observable is said to be free if it is not autonomous.

- $[11,25,36]$ A linear control system is said to be controllable if every observable is free.

- $[25,26,27]$ A linear control system $R y=0$ is parametrizable if there exist a matrix $R_{-1}$ with entries in $D$ and arbitrary functions $z$ such that the compatibility conditions of the inhomogeneous system $y=R_{-1} z$ are exactly generated by $R y=0$ or, equivalently, if there exists $R_{-1} \in D^{p \times m}$ such that $M=D^{1 \times p} / D^{1 \times q} R \cong D^{1 \times p} R_{-1}$. Then, $R_{-1}$ is called a parametrization of the system $R y=0$ and $z$ is a potential of the system.

- $[11,20,31]$ If a linear control system is parametrized by $R_{-1}$ and there exist a matrix $S_{-1}$ with entries in $D$ and $0 \neq \pi \in D$ satisfying $S_{-1} R_{-1}=\pi I$, where $I$ denotes the identity matrix, then the system is said to be $\pi$-free.

- $[11,20]$ A linear control system is flat (or free) if it is 1-free or, in other words, it is parametrizable and every component $z_{i}$ of the potential $z$ is an observable of the system or, equivalently, if there exists a parametrization $R_{-1} \in D^{p \times m}$ which admits a left-inverse $S_{-1} \in D^{m \times p}$, i.e., $S_{-1} R_{-1}=I_{m}$. Then, $z$ is called a flat output of the system.

We refer to [36] for a nice survey on the duality existing between the module-theoretic approach $[11,20,21,25,26,27,30]$ and the behavioural approach $[10,22,23,32,37]$ to multidimensional systems. This duality was firstly observed and developed by U. Oberst in [18]. Let us notice that research of parametrizations for multidimensional linear systems is called the image representation problem in the behavioural approach.

Proposition 4.2. Let $D$ be an Ore algebra, $R \in D^{q \times p}$ and $M=D^{1 \times p} / D^{1 \times q} R$ the left $D$-module associated with the system $R y=0$ (see Definition 3.1).

1. [25, 26, 36] The observables of the system are in a one-to-one correspondence with the left $D$-module $M$.

2. [11, 22, 25, 26, 36] The autonomous elements of the system are in a one-to-one correspondence with the torsion elements of $M$.

3. [11, 20, 22, 25, 26] The system is controllable iff $M$ is a torsion-free left D-module.

4. [11, 20] If $D$ is a commutative polynomial ring, then the system is $\pi$-free iff there exists a polynomial $0 \neq \pi \in D$ such that $D_{\pi} \otimes_{D} M=\left\{m / a \mid m \in M, a=\pi^{n}, n \in \mathbb{Z}_{\geq 0}\right\}$ is a free $D_{\pi}=$ $\left\{b / a \mid b \in D, a=\pi^{n}, n \in \mathbb{Z}_{\geq 0}\right\}$-module.

5. [11, 20] The system is flat iff $M$ is a free left D-module. Then, a basis of $M$ is a flat output of the system.

Let us recall the following well-known concepts of coprimeness developed in the literature of multidimensional systems. These concepts allow us to classify the systems.

Definition 4.3. $[11,18,27,37]$ Let $D=\mathbb{R}\left[x_{1}, \ldots, x_{n}\right]$ be a commutative polynomial ring, $R$ a full row rank matrix (namely, its rows are $D$-linearly independent) in $D^{q \times p}, J$ the ideal generated by the $q \times q$ minors of $R$ and $V(J)=\left\{\xi \in \mathbb{C}^{n} \mid \forall P \in J: P(\xi)=0\right\}$ the algebraic variety defined by $J$. Then,

- $R$ is called minor left-prime if $\operatorname{dim}_{\mathbb{C}} V(J) \leq n-1$, i.e., the greatest common divisor of all the $q \times q$ minors of $R$ is 1 .

- $R$ is called weakly zero left-prime if $\operatorname{dim}_{\mathbb{C}} V(J) \leq 0$, i.e., all the $q \times q$ minors of $R$ may only vanish simultaneously in a finite number of points of $\mathbb{C}^{n}$.

- $R$ is called zero left-prime if $\operatorname{dim}_{\mathbb{C}} V(J)=-1$, i.e., all the $q \times q$ minors of $R$ do not vanish simultaneously in $\mathbb{C}^{n}$.

Theorem 4.4. $[11,18,27]$ Let $D=\mathbb{R}\left[x_{1}, \ldots, x_{n}\right]$ be a commutative polynomial ring and $R$ a full row rank matrix in $D^{q \times p}$. Then, we have: 
1. $R$ is minor left-prime iff the $D$-module $M=D^{1 \times p} / D^{1 \times q} R$ is torsion-free.

2. If $n=3$, then $R$ is weakly zero left-prime iff the $D$-module $M=D^{1 \times p} / D^{1 \times q} R$ is reflexive.

3. $R$ is zero left-prime iff the $D$-module $M=D^{1 \times p} / D^{1 \times q} R$ is free.

Hence, the concepts of torsion-freeness, reflexiveness, projectiveness and freeness generalize to noncommutative polynomial rings the well-known concepts of primeness developed for multidimensional systems [27]. The next sections are dedicated to giving effective algorithms which check the module properties and compute the parametrizations and flat outputs.

\section{$5 \quad$ Syzygy modules and free resolutions}

Let $M$ be a finitely generated left module over a left noetherian ring $D$. We can reformulate the fact that $M$ is finitely generated by saying that there exists a surjective $D$-morphism $\varphi: D^{1 \times p} \rightarrow M$ which maps the $i$ th vector of the canonical basis $\left\{e_{i}\right\}_{1 \leq i \leq p}$ of $D^{1 \times p}$ to some $m_{i} \in M$. We have the exact sequence:

$$
\begin{array}{ccc}
D^{1 \times p} & \stackrel{\varphi}{\longrightarrow} M \\
e_{i} & \longmapsto & \longrightarrow
\end{array} m_{i} .
$$

This map can fail to be injective since there can be linear relations among the $\left\{m_{i}\right\}_{1 \leq i \leq p}$ :

$$
\operatorname{ker} \varphi=\left\{P=\left(P_{1}: \ldots: P_{p}\right) \in D^{1 \times p} \mid \varphi(P)=\sum_{i=1}^{p} P_{i} \varphi\left(e_{i}\right)=\sum_{i=1}^{p} P_{i} m_{i}=0\right\} .
$$

Definition 5.1. [33] The $D$-linear relations among the $m_{1}, \ldots, m_{p}$ form the left $D$-module $S(M)$ defined by (7) which is called the syzygy module of $M$ (this module is uniquely defined up to projective equivalence [33]). A similar definition exists for right $D$-modules.

Since $D$ is a left noetherian ring, $S(M)$ is a finitely generated left $D$-module. Therefore, we can again find a suitable free $D$-module $D^{1 \times q}$ and a map $\psi$ sending the canonical basis vectors of $D^{1 \times q}$ to a family of generators of $S(M)$ and we have the following exact sequence:

$$
D^{1 \times q} \stackrel{\psi}{\longrightarrow} D^{1 \times p} \stackrel{\varphi}{\longrightarrow} M \longrightarrow 0 .
$$

This exact sequence is called a finite presentation of the left $D$-module $M$ and $M$ is said to be finitely presented. Let us notice that, w.r.t. the canonical (standard) bases of $D^{1 \times q}$ and $D^{1 \times p}, \psi$ is defined by multiplication on the right with the matrix whose $i$ th row corresponds to the $i$ th generator of $S(M)$. Finally, iterating the preceding construction, we get the definition of a free resolution of the $D$-module $M$.

Definition 5.2. 1. [33] An exact sequence of the form

$$
\ldots \stackrel{d_{3}}{\longrightarrow} P_{2} \stackrel{d_{2}}{\longrightarrow} P_{1} \stackrel{d_{1}}{\longrightarrow} P_{0} \stackrel{d_{0}}{\longrightarrow} M \longrightarrow 0
$$

is called a (left/right) projective resolution of $M$ if the $D$-modules $P_{i}$ are projective (left/right) $D$-modules. The (left/right) $D$-module $S_{i}(M)=\operatorname{ker} d_{i}$ is called the $i$ th syzygy module of $M$.

2. [33] If the $D$-modules $P_{i}$ in (8) are free, then (8) is a free resolution of $M$.

3. [33] Let $0 \longrightarrow P_{n} \stackrel{d_{n}}{\longrightarrow} P_{n-1} \stackrel{d_{n-1}}{\longrightarrow} \ldots \stackrel{d_{2}}{\longrightarrow} P_{1} \stackrel{d_{1}}{\longrightarrow} P_{0} \stackrel{d_{0}}{\longrightarrow} M \longrightarrow 0$ be a projective resolution of $M$. We define the length of this resolution to be $n$.

4. [33] The minimal length of a left (resp. right) projective resolution of $M$ is called the left (resp. right) projective dimension $\operatorname{pd}_{D}\left({ }_{D} M\right)\left(\right.$ resp. $\left.\operatorname{pd}_{D}\left(M_{D}\right)\right)$ of the left (resp. right) $D$-module $M$. The (left/right) projective dimension can be infinite.

5. [33] We set $\operatorname{lgld} D=\sup \left\{\operatorname{pd}_{D}\left({ }_{D} M\right) \mid M\right.$ a left $D$-module $\} \in \mathbb{Z}_{\geq 0} \cup\{\infty\}$, which is called the left global dimension of $D$. Similarly for the right global dimension $\operatorname{rgld} D$. If $D$ is commutative, then we write gld $D=\operatorname{lgld} D=\operatorname{rgld} D$. 
We describe the computational tools for the construction of free resolutions. The techniques to compute syzygy modules use Gröbner bases and elimination techniques (see Section 6.1 of [2]). Let $D$ be an Ore algebra which satisfies (2) and $L$ a finitely generated left $D$-module which is a submodule of a free $D$-module $D^{1 \times p}, p \in \mathbb{N}$. Thus, a set of generators of $L$ consists of row vectors in $D^{1 \times p}$.

\section{Algorithm 5.3.}

Input: $\quad$ Set of generators $\left\{R_{1}, \ldots, R_{q}\right\} \subset D^{1 \times p}$ of the left $D$-module $L$, where $R_{i}=\left(R_{i 1}: \ldots: R_{i p}\right), i=1, \ldots, q$.

Output: $\quad S \in D^{r \times q}$ such that the left $D$-module $D^{1 \times r} S$ is the syzygy module $S(L)$ of $L$.

$\operatorname{SyzYgIES}\left(R_{1}, \ldots, R_{q}\right)$

Introduce the indeterminates $\lambda_{1}, \ldots, \lambda_{p}, \mu_{1}, \ldots, \mu_{q}$ over $D$.

$P \leftarrow\left\{\sum_{j=1}^{p} R_{i j} \lambda_{j}-\mu_{i} \mid i=1, \ldots, q\right\}$.

Compute the Gröbner basis $G$ of $P$ in the free left $D$-module generated by $\lambda_{1}, \ldots, \lambda_{p}, \mu_{1}, \ldots, \mu_{q}$, namely $\bigoplus_{i=1}^{p} D \lambda_{i} \oplus \bigoplus_{i=1}^{q} D \mu_{i}$, w.r.t. an order which eliminates the $\lambda_{i}$ 's.

$S=\left(S_{i j}\right) \in D^{r \times q} \leftarrow G \cap \bigoplus_{i=1}^{q} D \mu_{i}=\left\{\sum_{j=1}^{q} S_{i j} \mu_{j} \mid i=1, \ldots, r\right\}$.

Remark 5.4. Let us suppose that we have $R \in D^{q \times p}$ and the left $D$-module $M=D^{1 \times p} / D^{1 \times q} R$. Then, we can apply the preceding algorithm to the set formed by

$$
R_{i}=\left(R_{i 1}: \ldots: R_{i p}\right) \in L=D^{1 \times q} R \subseteq D^{1 \times p}, \quad i=1, \ldots, q,
$$

in order to obtain the matrix $S=\left(S_{i j}\right) \in D^{r \times q}$ such that

$$
S(M)=\operatorname{ker} . R=\left\{\left(P_{1}: \ldots: P_{q}\right) \in D^{1 \times q} \mid \sum_{i=1}^{q} P_{i} R_{i}=0\right\}=D^{1 \times r} S
$$

and we obtain the exact sequence $D^{1 \times r} \stackrel{. S}{\longrightarrow} D^{1 \times q} \stackrel{. R}{\longrightarrow} D^{1 \times p} \stackrel{\pi}{\longrightarrow} M \longrightarrow 0$. Iterating the process, we obtain a free resolution of the left $D$-module $M$.

For further developments and optimization of the technique, see [14].

Example 5.5. Let $D_{h}$ be the differential time-delay Ore algebra introduced in Example $3.2, R \in D_{h}^{3 \times 4}$ defined by (5) and the $D_{h}$-module $L=D_{h}^{1 \times 4} R^{T}$ generated by the rows of the matrix:

$$
R^{T}=\left(\begin{array}{ccc}
\partial+a & 0 & 0 \\
-k a \delta_{h} & \partial & \omega^{2} \\
0 & -1 & \partial+2 \zeta \omega \\
0 & 0 & -\omega^{2}
\end{array}\right) \in D_{h}^{4 \times 3} .
$$

We shall see later that the transposed matrix $R^{T}$ plays an important role in the characterization of the algebraic properties of the $D_{h}$-module $M=D_{h}^{1 \times 4} / D_{h}^{1 \times 3} R$. Let us compute the syzygy module of the $D_{h}$-module $L$. The reduced Gröbner basis of

$$
P=\left\{(\partial+a) \lambda_{1}-\mu_{1},-k a \delta_{h} \lambda_{1}+\partial \lambda_{2}+\omega^{2} \lambda_{3}-\mu_{2},-\lambda_{2}+(\partial+2 \zeta \omega) \lambda_{3}-\mu_{3},-\omega^{2} \lambda_{3}-\mu_{4}\right\}
$$

w.r.t. the elimination ordering induced by the degree reverse lexicographical orderings on $\lambda_{1}>\lambda_{2}>\lambda_{3}$ and $\mu_{1}>\mu_{2}>\mu_{3}>\mu_{4}>\delta_{h}>\partial$ resp. is:

$$
\begin{aligned}
G=\{ & \omega^{2} \lambda_{2}+\partial \mu_{4}+\omega^{2} \mu_{3}+2 \zeta \omega \mu_{4}, \omega^{2} k a \delta_{h} \lambda_{1}+\omega^{2} \mu_{2}+\omega^{2} \partial \mu_{3}+\left(\partial^{2}+2 \zeta \omega \partial+\omega^{2}\right) \mu_{4}, \\
& \omega^{2} k a \delta_{h} \mu_{1}+\left(\omega^{2} \partial+\omega^{2} a\right) \mu_{2}+\left(\omega^{2} \partial^{2}+\omega^{2} a \partial\right) \mu_{3}+\left(\partial^{3}+(2 \zeta \omega+a) \partial^{2}+\left(\omega^{2}+2 a \zeta \omega\right) \partial\right. \\
& \left.\left.+a \omega^{2}\right) \mu_{4},(\partial+a) \lambda_{1}-\mu_{1}, \omega^{2} \lambda_{3}+\mu_{4}\right\} .
\end{aligned}
$$

Intersecting $G$ with $\bigoplus_{i=1}^{4} D \mu_{i}$ we get

$$
\begin{aligned}
S=\left\{\omega^{2} k a \delta_{h} \mu_{1}+\left(\omega^{2} \partial+\omega^{2} a\right) \mu_{2}+\left(\omega^{2} \partial^{2}+\omega^{2} a \partial\right) \mu_{3}+\left(\partial^{3}+(2 \zeta \omega+a) \partial^{2}+\right.\right. & \\
& \left.\left.\left(\omega^{2}+2 a \zeta \omega\right) \partial+a \omega^{2}\right) \mu_{4}\right\} .
\end{aligned}
$$

If we denote by $R_{-1}^{T}$ the following row vector with entries in $D_{h}$

$$
R_{-1}^{T}=\left(\omega^{2} k a \delta_{h}: \omega^{2} \partial+\omega^{2} a: \omega^{2} \partial^{2}+\omega^{2} a \partial: \partial^{3}+(2 \zeta \omega+a) \partial^{2}+\left(\omega^{2}+2 a \zeta \omega\right) \partial+a \omega^{2}\right),
$$

then we obtain the following free resolution of the $D_{h}$-module $N=D_{h}^{1 \times 3} / D_{h}^{1 \times 4} R^{T}$ :

$$
0 \longrightarrow D_{h} \stackrel{. R_{-1}^{T}}{\longrightarrow} D_{h}^{1 \times 4} \stackrel{. R^{T}}{\longrightarrow} D_{h}^{1 \times 3} \stackrel{\pi}{\longrightarrow} N \longrightarrow 0 .
$$


We conclude this section by giving examples of left (right) global dimensions and by stating that free resolutions of finite length exist for every finitely generated module over Ore algebras $D=$ $A\left[\partial_{1} ; \sigma_{1}, \delta_{1}\right] \ldots\left[\partial_{m} ; \sigma_{m}, \delta_{m}\right]$, where all $\sigma_{i}$ are automorphisms. Note that every Ore algebra that we consider is of this kind (see e.g., Examples 2.3, 2.4, and 2.5).

Proposition 5.6. [17] Let $A$ be an integral domain with a finite left (resp. right) global dimension $\operatorname{lgld} A(r e s p . \operatorname{rgld} A)$ and $\sigma$ an automorphism of $A$.

1. The left (resp. right) global dimension of $A[\partial ; \sigma, \delta]$ satisfies:

$$
\operatorname{lgld} A \leq \operatorname{lgld} A[\partial ; \sigma, \delta] \leq \operatorname{lgld} A+1 \quad(\operatorname{rgld} A \leq \operatorname{rgld} A[\partial ; \sigma, \delta] \leq \operatorname{rgld} A+1) .
$$

If $\delta=0$, then we have:

$$
\operatorname{lgld} A[\partial ; \sigma, 0]=\operatorname{lgld} A+1 \quad(\operatorname{rgld} A[\partial ; \sigma, 0]=\operatorname{rgld} A+1) .
$$

2. If $k$ is a field, then we have $\operatorname{lgld} k\left[x_{1}, \ldots, x_{n}\right]=\operatorname{rgld} k\left[x_{1}, \ldots, x_{n}\right]=n$ and the Weyl algebra $A_{n}$ (see Example 2.3) satisfies $\operatorname{lgld} A_{n}=\operatorname{rgld} A_{n}=n$ if $k$ is a field of characteristic 0 and $\operatorname{lgld} A_{n}=\operatorname{rgld} A_{n}=2 n$ if $k$ is a field of characteristic $p>0$.

Example 5.7. By the preceding proposition, the Ore algebra $S_{h}$ of Example 2.4 has left (resp. right) global dimension 2 because lgld $k[t]=\operatorname{rgld} k[t]=1$. For the Ore algebra $D_{h}$ of Example 2.5, we have $\operatorname{lgld} D_{h}=\operatorname{rgld} D_{h}=2$ since $k[t]\left[\partial ; \sigma_{1}, \delta_{1}\right]=A_{1}$ has left (resp. right) global dimension 1 and $\delta_{2}=0$.

Let us notice that we do not know examples of rings satisfying $\operatorname{lgld} A[\partial ; \sigma, \delta]=\operatorname{lgld} A$.

Proposition 5.8. [17, 33] Let $D=A\left[\partial_{1} ; \sigma_{1}, \delta_{1}\right] \ldots\left[\partial_{m} ; \sigma_{m}, \delta_{m}\right]$ be an Ore algebra, where $\sigma_{i}$ is an automorphism, $i=1, \ldots, m$, and $M$ a finitely generated (left/right) $D$-module. Then, there is a free resolution of $M$ of length less than or equal $\operatorname{lgld} D+1($ resp. $\operatorname{rgld} D+1)$.

Proof. Iteratively applying Corollary 3.3 of [17] according to the construction of $D$ from the field $k$, it follows that every finitely generated projective (left/right) $D$-module $P$ is stably free. Since $D$ has finite (left/right) global dimension according to Proposition 5.6, $M$ has a projective resolution (8) of length less or equal to $\operatorname{lgld} D(\operatorname{resp} . \operatorname{rgld} D)$, where all $P_{i}$ are finitely generated projective, i.e., stably free (left/right) $D$-modules. Now, Lemma 9.40 of [33] states that, if $M$ has a stably free resolution of length $n$, namely a resolution of the form (8) where every $F_{i}$ is a stably free (left/right) $D$-module, then $M$ has a free resolution of length less or equal to $n+1$. Hence, we conclude that $M$ has a free resolution of length less or equal to $\operatorname{lgld} D+1($ resp. $\operatorname{rgld} D+1)$.

Let us notice that the previous result is a reminiscence of the concept of a Janet sequence developed in the theory of differential operators [25].

\section{Involutions}

Definition 6.1. Let $k$ be a field and $D$ a (non-commutative) $k$-algebra. An involution $\theta$ of $D$ is a $k$-linear map $\theta: D \rightarrow D$ satisfying

$$
\begin{aligned}
\theta\left(a_{1} \cdot a_{2}\right) & =\theta\left(a_{2}\right) \cdot \theta\left(a_{1}\right), \quad a_{1}, a_{2} \in D \\
\theta \circ \theta & =\operatorname{id}_{D},
\end{aligned}
$$

i.e., $\theta$ is an anti-automorphism of order two of the $k$-algebra $D$.

Proposition 6.2. By means of an involution $\theta$ of $D$, left $D$-modules can be turned into right $D$ modules and vice versa: let $D$ be a $k$-algebra, $M$ a right $D$-module and $\theta$ an involution of $D$, then we can define the left $D$-module $\widetilde{M}$, which is equal to $M$ as a set and which is endowed with the same addition as $M$, but with the following left action of $D$ :

$$
a m=m \theta(a), \quad m \in \widetilde{M}, \quad a \in D .
$$

Property (10) of $\theta$ ensures that $\widetilde{M}$ is a well-defined left D-module. 
Example 6.3. $\quad$ 1. Let $D$ be a commutative ring (e.g., $D=k\left[x_{1}, \ldots, x_{n}\right]$ ). Then, $\theta=i d_{D}$ is a trivial involution of $D$.

2. Let $A_{n}=k\left[x_{1}, \ldots, x_{n}\right]\left[\partial_{1} ; \sigma_{1}, \delta_{1}\right] \ldots\left[\partial_{n} ; \sigma_{n}, \delta_{n}\right]$ be the Weyl algebra (see Example 2.3). An involution $\theta$ of $A_{n}$ can be defined by $x_{i} \mapsto x_{i}, \quad \partial_{i} \mapsto-\partial_{i}, \quad 1 \leq i \leq n$.

3. Let $S_{h}=k[t]\left[\delta_{h} ; \sigma_{h}, \delta\right]$ be the shift Ore algebra (see Example 2.4). An involution $\theta$ of $S_{h}$ can be defined by $t \mapsto-t, \quad \delta_{h} \mapsto \delta_{h}$.

4. Let $H_{h}=k[t]\left[\partial ; \sigma_{1}, \delta_{1}\right]\left[\delta_{h} ; \sigma_{2}, \delta_{2}\right]\left[\tau_{h} ; \sigma_{3}, \delta_{3}\right]$ be the Ore algebra of differential time-delay and advance operators (see Example 2.5 for more details). An involution $\theta$ of $H_{h}$ can be defined by $t \mapsto t, \quad \partial \mapsto-\partial, \quad \delta_{h} \mapsto \tau_{h}, \quad \tau_{h} \mapsto \delta_{h}$.

5 . We can prove that there is no non-trivial involution for the Ore algebra $D_{h}$ of differential timedelay operators (see Example 2.5). Therefore, we need to embed $D_{h}$ into the Ore algebra $H_{h}$ of differential time-delay and advance operators.

Definition 6.4. Let $D$ be a left Ore algebra with an involution $\theta, R \in D^{q \times p}$ and $M=D^{1 \times p} / D^{1 \times q} R$ a left $D$-module.

- The dual $M^{*}$ of $M$ is the right $D$-module defined by $\operatorname{hom}_{D}(M, D)$.

- The transposed module of $M$ is the right $D$-module defined by:

$$
N=D^{q} / R D^{p} .
$$

If $D$ is a commutative ring, then we have $N=D^{1 \times q} / D^{1 \times p} R^{T}$, which explains the terminology.

- The adjoint module of $M$ is the left $D$-module defined by

$$
\widetilde{N}=D^{1 \times q} / D^{1 \times p} \theta(R)
$$

where $\theta(R)$ is the transpose of the matrix obtained by applying $\theta$ to each of its entries, i.e.:

$$
\theta(R)=\left(\theta\left(R_{i j}\right)\right)_{1 \leq i \leq q, 1 \leq j \leq p}^{T}
$$

Hence, the left $D$-module $\widetilde{N}=D^{1 \times q} / D^{1 \times p} \theta(R)$ corresponds to the linear system $\theta(R) z=0$, where $z=\left(z_{1}: \ldots: z_{q}\right)^{T}$.

Example 6.5. $\quad$ 1. If $D$ is a commutative ring (e.g., $D_{h}=\mathbb{R}(a, k, \zeta, \omega)\left[\partial ; \sigma_{1}, \delta_{1}\right]\left[\delta_{h} ; \sigma_{2}, \delta_{2}\right]$ defined in Example 3.2), then the involution $\theta$ is just the transposition of matrices, namely $\theta(R)=R^{T}$, and the transposed $D$-module is defined by $\widetilde{N}=D^{1 \times q} / D^{1 \times p} R^{T}=D^{q} / R D^{p}=N$.

2. Let us consider the Ore algebra $H_{h}=k[t]\left[\partial ; \sigma_{1}, \delta_{1}\right]\left[\delta_{h} ; \sigma_{2}, \delta_{2}\right]\left[\tau_{h} ; \sigma_{3}, \delta_{3}\right]$ defined in Example 2.5 and $R=\left[t \partial:-t^{2} \delta_{h}\right] \in H_{h}^{1 \times 2}$. Then, using 4 of Example 6.3, we obtain:

$$
\theta(R)=\left(\begin{array}{c}
-\partial t \\
-\tau_{h} t^{2}
\end{array}\right)=\left(\begin{array}{c}
-t \partial-1 \\
-(t+h)^{2} \tau_{h}
\end{array}\right) \in H_{h}^{2 \times 1} .
$$

\section{Computation of extension modules}

Definition 7.1. [33] Let $M$ be a finitely generated left $D$-module, $M^{\prime}$ a left $D$-module and

$$
\text { .. } \stackrel{d_{2}}{\longrightarrow} F_{1} \stackrel{d_{1}}{\longrightarrow} F_{0} \stackrel{d_{0}}{\longrightarrow} M \longrightarrow 0
$$

a free resolution of $M$. Then, the defects of exactness of the complex

$$
\ldots \longleftarrow \operatorname{hom}_{D}\left(F_{2}, M^{\prime}\right) \stackrel{d_{2}^{*}}{\longleftarrow} \operatorname{hom}_{D}\left(F_{1}, M^{\prime}\right) \stackrel{d_{1}^{*}}{\longleftarrow} \operatorname{hom}_{D}\left(F_{0}, M^{\prime}\right) \longleftarrow 0,
$$

where $d_{i}^{*}$ is defined by $d_{i}^{*}(f)=f \circ d_{i}$ for $f \in \operatorname{hom}_{D}\left(F_{i-1}, M^{\prime}\right), i \geq 1$, are defined by the abelian groups:

$$
\left\{\begin{array}{l}
\operatorname{ext}_{D}^{0}\left(M, M^{\prime}\right)=\operatorname{ker} d_{1}^{*}=\operatorname{hom}_{D}\left(M, M^{\prime}\right), \\
\operatorname{ext}_{D}^{i}\left(M, M^{\prime}\right)=\operatorname{ker} d_{i+1}^{*} / \operatorname{im} d_{i}^{*}, \quad i \geq 1 .
\end{array}\right.
$$


In the following, we shall only consider the case $M^{\prime}=D$, and thus, $\operatorname{ext}_{D}^{i}(M, D), i \in \mathbb{Z}_{\geq 0}$. Let us notice that $\operatorname{ext}_{D}^{i}(M, D), i \in \mathbb{Z}_{\geq 0}$, inherit a right module structure by the right action of $D$.

Proposition 7.2. [33] The right $D$-module $\operatorname{ext}_{D}^{i}(M, D)$ only depends on $M$, i.e., we can choose any free resolution of $M$ to compute $\operatorname{ext}_{D}^{i}(M, D), i \in \mathbb{Z}_{\geq 0}$.

The next algorithm gives a description of the left $D$-module $\widetilde{\operatorname{ext}_{D}^{1}(M, D)}$, which corresponds to the right $D$-module $\operatorname{ext}_{D}^{1}(M, D)$ (see Proposition 6.2).

\section{Algorithm 7.3.}

Input: $\quad$ Ore algebra $D$ satisfying (2) with an involution $\theta$ and $R=\left(R_{1}^{T}: \ldots: R_{q}^{T}\right)^{T} \in D^{q \times p}$.

Output: A list $L=\left(L_{1}, L_{2}\right)$ of two matrices such that:

$L_{1} \in D^{m \times q}$ is such that $\widetilde{\operatorname{ext}_{D}^{1}(M, D)}=D^{1 \times m} L_{1} / D^{1 \times p} \theta(R)$, where $M=D^{1 \times p} / D^{1 \times q} R$, $L_{2} \in D^{q \times r}$ is such that $L_{1}=\operatorname{SYZYgies}\left(L_{2}\right)$.

$\operatorname{ADJEXT} 1(R)$

$R_{2} \leftarrow \operatorname{SyZYgIES}(R)$

$L_{2} \leftarrow \theta\left(R_{2}\right)$,

$L_{1} \leftarrow \operatorname{SYZYGIES}\left(L_{2}\right)$,

$L \leftarrow\left(L_{1}, L_{2}\right)$.

Proof. The module $M$ is given as the cokernel $D^{1 \times p} / D^{1 \times q} R$ of the $D$-linear map:

$$
D^{1 \times q} \stackrel{\cdot R}{\longrightarrow} D^{1 \times p} .
$$

Computing the first syzygy module of $M$, we obtain a matrix $R_{2} \in D^{r \times q}$ such that the sequence

$$
D^{1 \times r} \stackrel{. R_{2}}{\longrightarrow} D^{1 \times q} \stackrel{. R}{\longrightarrow} D^{1 \times p}
$$

is exact. Upon dualization, we get the complex

$$
D^{r \times 1} \stackrel{R_{2}}{\longleftarrow} D^{q \times 1} \stackrel{R .}{\longleftarrow} D^{p \times 1}
$$

with defect of exactness $\operatorname{ext}_{D}^{1}(M, D)=\operatorname{ker}\left(R_{2}.\right) / R D^{p \times 1}$ at $D^{q \times 1}$. Taking adjoints and writing $L_{2}=$ $\theta\left(R_{2}\right) \in D^{q \times r}$, we obtain the complex

$$
D^{1 \times r} \stackrel{. L_{2}}{\longleftarrow} D^{1 \times q} \stackrel{. \theta(R)}{\longleftarrow} D^{1 \times p}
$$

with defect of exactness $\operatorname{ext}_{D}^{1(M, D)}$. Computing the first syzygy module of coker(.. $\left.L_{2}\right)$ yields the exact sequence

$$
D^{1 \times r} \stackrel{. L_{2}}{\longleftarrow} D^{1 \times q} \stackrel{. L_{1}}{\longleftarrow} D^{1 \times m}
$$

such that:

$$
\widetilde{\operatorname{ext}_{D}^{1}(M, D)}=\operatorname{ker}\left(. L_{2}\right) / D^{1 \times p} \theta(R)=D^{1 \times m} L_{1} / D^{1 \times p} \theta(R)
$$

Example 7.4. Let us compute $\operatorname{ext}_{D_{h}}^{i}\left(N, D_{h}\right)$ of the $D_{h}$-module $N$ defined in Example 5.5. In Example 5.5, we have already computed the free resolution (9) of $N=D_{h}^{1 \times 3} / D_{h}^{1 \times 4} R^{T}$. Thus, we have ker. $R^{T}=D_{h} R_{-1}^{T}$, where $R_{-1}^{T}$ is defined in Example 5.5. Then, using the fact that $D_{h}=$ $\mathbb{R}(a, k, \zeta, \omega)\left[\partial ; \sigma_{1}, \delta_{1}\right]\left[\delta_{h} ; \sigma_{2}, \delta_{2}\right]$ is a commutative polynomial ring, we obtain that $\theta\left(R_{-1}^{T}\right)=R_{-1}$ (see 1 of Example 6.3). Hence, we have the following complex

$$
0 \longleftarrow D_{h} \stackrel{\cdot R_{-1}}{\longleftarrow} D_{h}^{1 \times 4} \stackrel{. R}{\longleftarrow} D_{h}^{1 \times 3} \longleftarrow 0
$$

and its defects of exactness are defined by:

$$
\begin{aligned}
& \operatorname{ext}_{D_{h}}^{1}\left(N, D_{h}\right)=\operatorname{ker} \cdot R_{-1} / D_{h}^{1 \times 3} R, \\
& \operatorname{ext}_{D_{h}}^{2}\left(N, D_{h}\right)=D_{h} / D_{h}^{1 \times 4} R_{-1} .
\end{aligned}
$$


Following Algorithm 7.3, we need to compute the syzygy module of $D_{h}^{1 \times 4} R_{-1}$. The reduced Gröbner basis of

$$
\begin{aligned}
P=\{ & \left(\omega^{2} k a \delta_{h}\right) \lambda-\mu_{1},\left(\omega^{2} \partial+\omega^{2} a\right) \lambda-\mu_{2},\left(\omega^{2} \partial^{2}+\omega^{2} a \partial\right) \lambda-\mu_{3}, \\
& \left.\left(\partial^{3}+2 \zeta \omega \partial^{2}+a \partial^{2}+\omega^{2} \partial+2 a \zeta \omega \partial+a \omega^{2}\right) \lambda-\mu_{4}\right\}
\end{aligned}
$$

w.r.t. the elimination ordering induced by the degree reverse lexicographical orderings on $\lambda$ and $\mu_{1}>\mu_{2}>\mu_{3}>\mu_{4}>\delta_{h}>\partial$ resp. is:

$$
\begin{aligned}
G=\left\{(\partial+a) \mu_{1}-k a \delta \mu_{2}, \omega^{2} \mu_{2}+(\partial+2 \zeta \omega) \mu_{3}-\omega^{2} \mu_{4}, \partial \mu_{2}-\mu_{3},\right. \\
\left.\omega^{2}(\partial+a) \lambda-\mu_{2}, \omega^{2} k a \delta \lambda-\mu_{1}\right\} .
\end{aligned}
$$

Therefore, we obtain that the syzygy module of $D_{h}^{1 \times 4} R_{-1}$ is defined by the matrix

$$
L=\left(\begin{array}{cccc}
\partial+a & -k a \delta_{h} & 0 & 0 \\
0 & \omega^{2} & \partial+2 \zeta \omega & -\omega^{2} \\
0 & \partial & -1 & 0
\end{array}\right) \in D_{h}^{3 \times 4},
$$

and thus, we have $\operatorname{ext}_{D_{h}}^{1}\left(N, D_{h}\right)=D_{h}^{1 \times 3} L / D_{h}^{1 \times 3} R$. Finally, using (12), $\operatorname{ext}_{D_{h}}^{2}\left(N, D_{h}\right)$ corresponds to the system associated with $R_{-1} z=0$, namely:

$$
\left\{\begin{array}{l}
\left(\omega^{2} k a \delta_{h}\right) y=0 \\
\left(\omega^{2} \partial+\omega^{2} a\right) y=0 \\
\left(\omega^{2} \partial^{2}+\omega^{2} a \partial\right) y=0 \\
\left(\partial^{3}+(2 \zeta \omega+a) \partial^{2}+\left(\omega^{2}+2 a \zeta \omega\right) \partial+a \omega^{2}\right) y=0 .
\end{array}\right.
$$

Note that $\operatorname{ext}_{D_{h}}^{2}\left(N, D_{h}\right) \neq 0$ because, otherwise, in $G$, we would have had $\lambda-\sum_{i=1}^{4} P_{i} \mu_{i}$, for some $P_{i} \in D_{h}$.

The quotient module $\operatorname{ext}_{D}^{1(M, D)}=D^{1 \times m} L_{1} / D^{1 \times p} \theta(R)$ can be computed using elimination techniques similar to Algorithm 5.3.

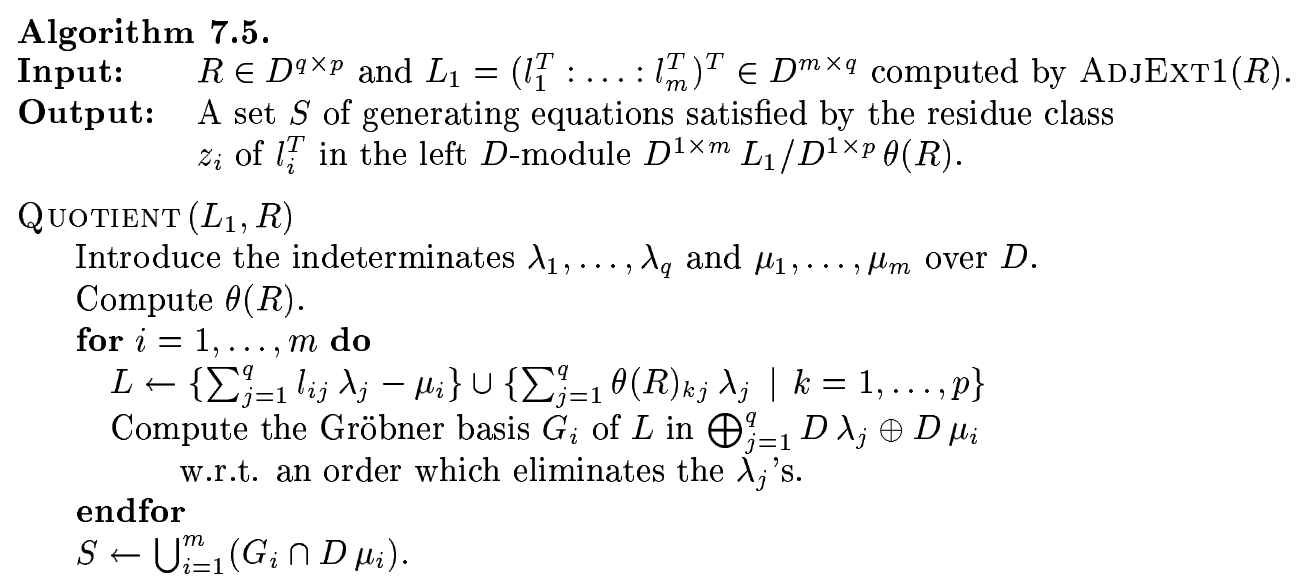

Remark 7.6. In the result of the preceding algorithm, each $G_{i} \cap D \mu_{i}$ is a generating set of the relations fulfilled by $z_{i}$. Let us notice that every polynomial in $G_{i} \cap D \mu_{i}$ has the form $P \mu_{i}$, for a certain $P \in D$.

Example 7.7. In Example 7.4, we proved that $\operatorname{ext}_{D_{h}}^{1}\left(N, D_{h}\right)=D_{h}^{1 \times 3} L / D_{h}^{1 \times 3} R$, where $R$ (resp. $L$ ) is defined by (5) (resp. (13)), $N=D_{h}^{1 \times 3} / D_{h}^{1 \times 4} R^{T}$ and $D_{h}=\mathbb{R}(a, k, \zeta, \omega)\left[\partial ; \sigma_{1}, \delta_{1}\right]\left[\delta_{h} ; \sigma_{2}, \delta_{2}\right]$. In order to check whether or not $\operatorname{ext}_{D_{h}}^{1}\left(N, D_{h}\right)$ is equal to 0 , we apply $\operatorname{QuOTIENT}(L, R)$ described in Algorithm 7.5. If we denote

$$
R=\left(R_{1}^{T}: R_{2}^{T}: R_{3}^{T}\right)^{T}, \quad L=\left(l_{1}^{T}: l_{2}^{T}: l_{3}^{T}\right)^{T},
$$

then we easily check that we have $G_{i} \cap D \mu_{i}=\left\{\mu_{i}\right\}$, for $i=1, \ldots, 3$, because we have $l_{1}=R_{1}, l_{2}=R_{3}$, $l_{3}=R_{2}$. Hence, we have $D_{h}^{1 \times 3} L=D_{h}^{1 \times 3} R$, which shows that $\operatorname{ext}_{D_{h}}^{1}\left(N, D_{h}\right)=D_{h}^{1 \times 3} L / D_{h}^{1 \times 3} R=0$.

The next algorithm gives a description of $\widetilde{\operatorname{ext}_{D}^{1}(M, D)}=D_{h}^{1 \times m} L_{1} / D_{h}^{1 \times p} \theta(R)$ in which the quotient is explicitly computed. 
Algorithm 7.8.

Input: An Ore algebra $D$ satisfying (2) with an involution $\theta$ and $R=\left(R_{1}^{T}: \ldots: R_{q}^{T}\right)^{T} \in D^{q \times p}$.

Output: A tuple $L=\left(L_{0}, L_{1}, L_{2}\right)$ such that:

$L_{1}=\left(l_{1}^{T}: \ldots: l_{m}^{T}\right)^{T} \in D^{m \times q}$ is such that

$\operatorname{ext}_{D}^{1}(M, D)=D^{1 \times m} L_{1} / D^{1 \times p} \theta(R)$, where $M=D^{1 \times p} / D^{1 \times q} R$,

$L_{2} \in D^{q \times r}$ is such that $L_{1}=\operatorname{Syzygies}\left(L_{2}\right)$,

$L_{0}$ is the set of the relations satisfied by the residue class $z_{i}$

of $l_{i}^{T}$ in the left $D$-module $D^{1 \times m} L_{1} / D^{1 \times p} \theta(R)$.

$\operatorname{ExT} 1(R)$

$\left(L_{1}, L_{2}\right) \leftarrow \operatorname{ADJExT}(R)$,

$L_{0} \leftarrow$ Quotient $\left(L_{1}, R\right)$,

$L \leftarrow\left(L_{0}, L_{1}, L_{2}\right)$.

Remark 7.9. First of all, if $L_{0}$ is an identity matrix, then we have $\operatorname{ext}_{D}^{1}(M, D)=0$ (e.g., see Example 7.7). Secondly, let us notice that while using ExT1, we start to compute a free resolution of the left $D$-module $M$ (see Algorithm 7.3). If we denote this resolution by

$$
\ldots \stackrel{. R_{3}}{\longrightarrow} D^{1 \times p_{2}} \stackrel{. R_{2}}{\longrightarrow} D^{1 \times p_{1}} \stackrel{. R_{1}}{\longrightarrow} D^{1 \times p_{0}} \stackrel{\pi}{\longrightarrow} M \longrightarrow 0,
$$

where $p_{0}=p, p_{1}=q$ and $R_{1}=R$, then $\operatorname{Ext} 1\left(R_{i}\right)$ computes $\widetilde{\operatorname{ext}_{D}^{i}(M, D)}, i \geq 1$.

\section{Characterization of module properties}

The main motivation for introducing the concept of extension functor is explained in the next theorems which give an effective way to check the module properties defined in Section 3, and thus, the structural properties of the corresponding linear control system (see Section 4).

Definition 8.1. Let $A$ and $B$ be two rings and $M$ an abelian group. $M$ is said to be an $A$-B-bimodule, denoted ${ }_{A} M_{B}$ if $M$ is a left $A$-module and a right $B$-module and the two actions are related by the following associative law $\forall a \in A, \forall b \in B, \forall m \in M, a(m b)=(a m) b$.

In this section, $D$ is supposed to be a left and a right noetherian integral domain having the left and right Ore properties (see Proposition 2.7). Let us recall a classical result in homological algebra saying that a short exact sequence of bimodules gives rise to a long exact sequence of the cohomology right modules.

Lemma 8.2. [33] Let $N$ be a left $D$-module and $0 \longrightarrow A \longrightarrow B \longrightarrow C \longrightarrow 0$ an exact sequence of $D$-D-bimodules. Then, we have the following exact sequence of right $D$-modules:

$$
\begin{aligned}
& 0 \longrightarrow \operatorname{hom}_{D}(N, A) \longrightarrow \operatorname{hom}_{D}(N, B) \longrightarrow \operatorname{hom}_{D}(N, C) \\
& \longrightarrow \operatorname{ext}_{D}^{1}(N, A) \longrightarrow \operatorname{ext}_{D}^{1}(N, B) \longrightarrow \operatorname{ext}_{D}^{1}(N, C) \\
& \longrightarrow \operatorname{ext}_{D}^{2}(N, A) \quad \longrightarrow \quad \ldots
\end{aligned}
$$

Theorem 8.3. [29] Let $M$ be a left D-module defined by the finite presentation

$$
F_{1} \stackrel{d_{1}}{\longrightarrow} F_{0} \longrightarrow M \longrightarrow 0,
$$

and the right $D$-module $N$ the transposed module of $M$ defined by:

$$
0 \longleftarrow N \longleftarrow F_{1}^{*} \stackrel{d_{1}^{*}}{\longleftarrow} F_{0}^{*} \longleftarrow M^{*} \longleftarrow 0 .
$$

Then $t(M) \cong \operatorname{ext}_{D}^{1}(N, D)$. In particular, $M$ is a torsion-free left $D$-module iff $\operatorname{ext}_{D}^{1}(N, D)=0$.

Proof. Let $X$ be a $D$-D-bimodule. Then, applying the right exact functor $X \otimes_{D} \cdot[4,33]$ to the exact sequence (15) gives the exact sequence

$$
X \otimes_{D} F_{1} \stackrel{\mathrm{id}_{X} \otimes d_{1}}{\longrightarrow} X \otimes_{D} F_{0} \longrightarrow X \otimes_{D} M \longrightarrow 0
$$


whereas applying the left exact functor $\operatorname{hom}(\cdot, X)$ to the exact sequence (16) yields the exact sequence:

$$
0 \longrightarrow \operatorname{hom}_{D}(N, X) \longrightarrow \operatorname{hom}_{D}\left(F_{1}^{*}, X\right) \longrightarrow \operatorname{hom}_{D}\left(F_{0}^{*}, X\right) .
$$

Using that the $F_{i}$ are finitely generated free left $D$-modules, we have $\operatorname{hom}_{D}\left(F_{i}^{*}, X\right) \cong X \otimes_{D} F_{i}[33]$, for $i=1,2$, and thus, we finally obtain the following exact sequence:

$$
0 \longrightarrow \operatorname{hom}_{D}(N, X) \longrightarrow X \otimes_{D} F_{1} \longrightarrow X \otimes_{D} F_{0} \longrightarrow X \otimes_{D} M \longrightarrow 0 .
$$

Now, let $K=Q(D)$ be the quotient field of $D$ with its bimodule structure and let us consider the exact sequence of $D$-D-bimodules:

$$
0 \longrightarrow D \longrightarrow K \longrightarrow K / D \longrightarrow 0 .
$$

Using Lemma 8.2 with $A=D, B=K, C=K / D$, we get the exact sequence of right $D$-modules

$$
0 \longrightarrow \operatorname{hom}_{D}(N, D) \longrightarrow \operatorname{hom}_{D}(N, K) \longrightarrow \operatorname{hom}_{D}(N, K / D) \longrightarrow \operatorname{ext}_{D}^{1}(N, D) \longrightarrow 0
$$

because $\operatorname{ext}_{D}^{1}(N, K)=0$ since $K$ is a left injective $D$-module (see [33] for more details).

Moreover, by applying the right exact functor $\cdot \otimes_{D} M$ to the short exact sequence (18), we obtain the exact sequence of left $D$-modules

$$
M \stackrel{i_{K}}{\longrightarrow} K \otimes_{D} M \longrightarrow(K / D) \otimes_{D} M \longrightarrow 0,
$$

which extends to the following exact sequence:

$$
0 \longrightarrow t(M) \longrightarrow M \stackrel{i_{K}}{\longrightarrow} K \otimes_{D} M \longrightarrow(K / D) \otimes_{D} M \longrightarrow 0,
$$

where the kernel of the $D$-morphism $i_{K}$, defined by $\left\{m \in M \mid i_{K}(m)=1 \otimes m=0\right\}$, is the left submodule of $M$ formed by all elements $m \in M$ for which there exists $0 \neq d \in D$ such that $d m=0$, i.e., the torsion submodule $t(M)$ of $M$ [33].

Using the fact that the $F_{i}$ are finitely generated free left $D$-modules, and thus, flat left $D$-modules $[4,33]$, by applying $\cdot \otimes_{D} F_{i}$ to the exact sequence (18), we obtain the exact sequence:

$$
0 \longrightarrow F_{i} \longrightarrow K \otimes_{D} F_{i} \longrightarrow(K / D) \otimes_{D} F_{i} \longrightarrow 0 .
$$

Finally, combining together the exact sequences (17) for $X=D, K$ and $K / D,(19),(20)$ and (21), we obtain the following commutative exact diagram:

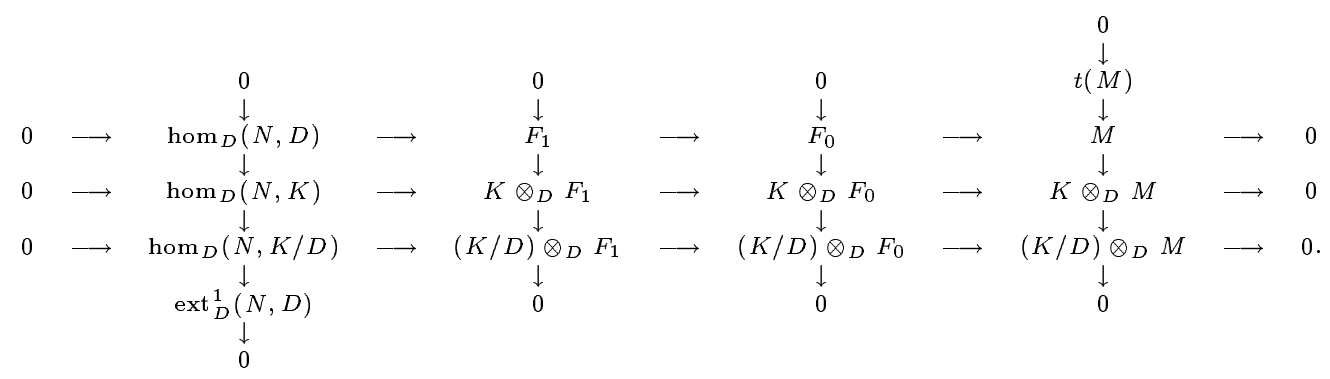

Then, an easy chase in the previous commutative exact diagram shows that $t(M) \cong \operatorname{ext}_{D}^{1}(N, D)$.

Lemma 8.4. [4] If $M_{1} \stackrel{\alpha_{1}}{\longrightarrow} M_{2} \stackrel{\alpha_{2}}{\longrightarrow} M_{3} \stackrel{\alpha_{3}}{\longrightarrow} M_{4}$ is a complex of (left/right) D-modules, then we have an exact ker-coker sequence induced by the map $\alpha_{2}$

$$
0 \longrightarrow H\left(M_{2}\right) \longrightarrow \text { coker } \alpha_{1} \longrightarrow \operatorname{ker} \alpha_{3} \longrightarrow \operatorname{ker} H\left(M_{3}\right) \longrightarrow 0
$$

where $H\left(M_{i}\right)$ is the defect of exactness of the complex at $M_{i}$, namely $H\left(M_{i}\right)=\operatorname{ker} \alpha_{i} / \mathrm{im} \alpha_{i-1}, i=2,3$, and coker $\alpha_{1}=M_{2} / \mathrm{im} \alpha_{1}$.

Theorem 8.5. Let $M$ be a finitely presented left D-module, i.e., defined by the finite presentation (15), and $N$ the transposed module of $M$ defined by the finite presentation (16). Then, we have the following exact sequence:

$$
0 \longrightarrow \operatorname{ext}_{D}^{1}(N, D) \longrightarrow M \stackrel{\varepsilon_{M}}{\longrightarrow} M^{* *} \longrightarrow \operatorname{ext}_{D}^{2}(N, D) \longrightarrow 0 .
$$

Hence, $M$ is a reflexive left $D$-module iff $\operatorname{ext}_{D}^{i}(N, D)=0, i=1,2$. 
Proof. Let (15) be a finite presentation of $M$. Then, its transposed module is defined by $N=$ coker $d_{1}^{*}$ in the exact sequence (16). We extend this finite presentation to an exact sequence by computing the second and the third syzygy module of $N$. We obtain the following exact sequence:

$$
0 \longleftarrow N \longleftarrow F_{1}^{*} \stackrel{d_{1}^{*}}{\longleftarrow} F_{0}^{*} \stackrel{d_{0}^{*}}{\longleftarrow} F_{-1}^{*} \stackrel{d_{-1}^{*}}{\longleftarrow} F_{-2}^{*} .
$$

Dualizing the exact sequence (23) and using the fact that $F_{i}$ is canonically isomorphic to $F_{i}^{* *}$ because the $F_{i}$ are finitely generated free left $D$-modules, we obtain the following complex

$$
F_{1} \stackrel{d_{1}}{\longrightarrow} F_{0} \stackrel{d_{0}}{\longrightarrow} F_{-1} \stackrel{d_{-1}}{\longrightarrow} F_{-2} .
$$

Now, applying Lemma 8.4 to the complex (24) and using the definitions of $\operatorname{ext}_{D}^{1}(N, D)$ and $\operatorname{ext}_{D}^{2}(N, D)$, we obtain the exact sequence:

$$
0 \longrightarrow \operatorname{ext}_{D}^{1}(N, D) \longrightarrow \operatorname{coker} d_{1} \longrightarrow \operatorname{ker} d_{-1} \longrightarrow \operatorname{ext}_{D}^{2}(N, D) \longrightarrow 0 .
$$

According to the presentation of $M$, we have $M=$ coker $d_{1}$. Now, using the exact sequences (16) and (23), we obtain the following exact sequence:

$$
0 \longleftarrow M^{*}=\operatorname{ker} d_{1}^{*} \longleftarrow F_{-1}^{*} \stackrel{d_{-1}^{*}}{\longleftarrow} F_{-2}^{*} .
$$

By applying the left exact functor $\operatorname{hom}_{D}(\cdot, D)$ to $(25)[4,33]$, we obtain the following exact sequence

$$
0 \longrightarrow M^{* *} \longrightarrow F_{-1} \stackrel{d_{-1}}{\longrightarrow} F_{-2},
$$

proving that $M^{* *}=\operatorname{ker} d_{-1}$. Finally, we have the exact sequence (22), which proves that $M \cong M^{* *}$ iff $\operatorname{ext}_{D}^{i}(N, D)=0, i=1,2$.

Corollary 8.6. Let $M$ be a finitely generated left $D$-module satisfying $\operatorname{hom}_{D}(M, D)=0$. Then, $M$ is a torsion left $D$-module.

Proof. If $M^{*}=\operatorname{hom}_{D}(M, D)=0$, then $M^{* *}=\operatorname{hom}_{D}\left(M^{*}, D\right)=0$. Then, using the exact sequence (22), we obtain that $\operatorname{ext}_{D}^{1}(N, D)=\operatorname{ker} \varepsilon_{M}=M$. Finally, using Theorem 8.3, we obtain that $t(M)=$ $\operatorname{ext}_{D}^{1}(N, D)=M$, i.e., $M$ is a torsion left $D$-module.

Theorem 8.7. Let us suppose that $\operatorname{rgld} D=n<+\infty$ and let $M$ be a left $D$-module defined by the finite presentation (15) and $N$ its transposed module defined by the finite presentation (16). Then, $M$ is a projective left $D$-module iff $\operatorname{ext}_{D}^{i}(N, D)=0, i=1, \ldots, \operatorname{rgld} D$.

Proof. $\Rightarrow$ Let us suppose that $M$ is a projective left $D$-module. In particular, $M$ is a reflexive left $D$-module, and thus, by Theorem 8.5, we have $\operatorname{ext}_{D}^{i}(N, D)=0, i=1,2$.

Let us notice that from the exact sequence $(16)$, we deduce $[4,33]$ :

$$
\operatorname{ext}_{D}^{i+2}(N, D) \cong \operatorname{ext}_{D}^{i}\left(M^{*}, D\right), \quad i \geq 1 .
$$

Now, using the fact that $M$ is a projective left $D$-module, we obtain that $M^{*}$ is a projective right $D$-module. Therefore, we have $\operatorname{ext}_{D}^{i}\left(M^{*}, D\right)=0, i \geq 1[4,33]$ which implies that $\operatorname{ext}_{D}^{i}(N, D)=0$, $i \geq 3$, and finally proves the first implication.

$\Leftarrow$ Let us suppose that $\operatorname{ext}_{D}^{i}(N, D)=0, i=1, \ldots, \operatorname{rgld} D$. Let us consider a projective resolution of length $\operatorname{rgld} D$ of the right $D$-module $N$ :

$$
0 \longleftarrow N \longleftarrow P_{0} \stackrel{r_{1}}{\longleftarrow} P_{1} \stackrel{r_{2}}{\longleftarrow} P_{2} \stackrel{r_{3}}{\longleftarrow} \ldots \stackrel{r_{\mathrm{rgld} D}}{\longleftarrow} P_{\mathrm{rgld} D} \longleftarrow 0
$$

Dualizing (26) and using the fact that $\operatorname{ext}_{D}^{i}(N, D)=0, i=1, \ldots, \operatorname{rgld} D$, we obtain that the following complex

$$
0 \longrightarrow N^{*} \longrightarrow P_{0}^{*} \stackrel{r_{1}^{*}}{\longrightarrow} P_{1}^{*} \stackrel{r_{2}^{*}}{\longrightarrow} P_{2}^{*} \stackrel{r_{3}^{*}}{\longrightarrow} \ldots \stackrel{r_{\mathrm{rgld} D}^{*}}{\longrightarrow} P_{\mathrm{rgld} D}^{*} \longrightarrow 0
$$

is an exact sequence. The dual of a projective $D$-module is also a projective $D$-module $[4,33]$, and thus, $P_{i}^{*}$ is a projective left $D$-module for $i=0, \ldots, r_{\mathrm{rgld}} D$. Moreover, the exact sequence (27) ends with a projective left $D$-module $P_{\mathrm{rgld}}^{*}$, and thus, (27) is a split exact sequence (see Proposition 3.8). 
Therefore, by induction, we deduce that im $r_{i}^{*}$ is a projective left $D$-module for $i=1, \ldots, \operatorname{rgld} D$, and thus, we obtain the split exact sequence

$$
0 \longrightarrow N^{*} \longrightarrow P_{0}^{*} \longrightarrow \mathrm{im} r_{1}^{*} \longrightarrow 0
$$

which shows that $P_{0}^{*} \cong N^{*} \oplus \operatorname{im} r_{1}^{*}$, i.e., $N^{*}$ is a projective left $D$-module. Hence, the right $D$-module $N^{* *}$ is also projective. Dualizing again the split exact sequence (27) and using the fact that the $P_{i}$ are projective $D$-modules, and thus, reflexive, namely $P_{i}^{* *} \cong P_{i}$, we obtain the split exact sequence

$$
0 \longleftarrow N^{* *} \longleftarrow P_{0} \stackrel{r_{1}}{\longleftarrow} P_{1} \stackrel{r_{2}}{\longleftarrow} P_{2} \stackrel{r_{3}}{\longleftarrow} \ldots \stackrel{r_{\mathrm{rgld} D}}{\longleftarrow} P_{\operatorname{rgld} D} \longleftarrow 0
$$

from which we deduce that $N \cong N^{* *}$, and thus, $N$ is a projective right $D$-module. The exact sequence (16) ends with the projective right $D$-module $N$, and thus, (16) is a split exact sequence (see Proposition 3.8). Thus, we deduce that $M^{*}$ is a projective right $D$-module, which implies that $M^{* *}$ is a projective left $D$-module. Finally, using the fact that $\operatorname{ext}_{D}^{i}(N, D)=0, i=1,2$, by Theorem 8.5 , we obtain that $M \cong M^{* *}$, and thus, $M$ is a projective left $D$-module.

Corollary 8.8. Let $M=D^{1 \times p} / D^{1 \times q} R$ be a left $D$-module and $\widetilde{N}=D^{1 \times q} / D^{1 \times p} \theta(R)$ the transposed module of $M$. Then, we have the following equivalences:

1. The system $R y=0$ is parametrizable iff $t(M) \cong \operatorname{ext}_{D}^{1}(N, D)=0$. Then, the matrix $L_{2}$ in $\operatorname{ExT} 1(R)$ is a parametrization of the system $R y=0$.

2. If $R$ has full row rank, namely $S\left(D^{1 \times q} R\right)=0$, then the following assertions are equivalent:

(a) $M$ is a projective left $D$-module,

(b) $\exists S \in D^{p \times q}: R S=I_{q}$,

(c) $N=\operatorname{ext}_{D}^{1}(M, D)=0$.

Proof. 1. $\Rightarrow$ Let us suppose that $R y=0$ is parametrizable. Then there exists $R_{-1} \in D^{p \times m}$ such that $M=D^{1 \times p} / D^{1 \times q} R \cong D^{1 \times p} R_{-1} \subseteq D^{1 \times m}$. Therefore, the left $D$-module $M$ is isomorphic to a left submodule $\phi(M)$ of $D^{1 \times m}$, where $\phi$ is the isomorphism between $M$ and $D^{1 \times p} R_{-1}$. Let us suppose that there exists a non-zero torsion element $z \in M$ satisfying $d z=0$, where $0 \neq d \in D$. Then, we have $\phi(d z)=0$, and thus, using the fact that $\phi$ is a $D$-morphism, then we obtain $d \phi(z)=0$, i.e., $\phi(z)$ is a torsion element of $D^{1 \times m}$. But, $D^{1 \times m}$ is a free left $D$-module, and thus, $t\left(D^{1 \times m}\right)=0$, which proves that $t(M) \cong \operatorname{ext}_{D}^{1}(N, D)=0$ (or, equivalently, if $\left\{e_{i}\right\}_{1 \leq i \leq m}$ is a basis of $D^{1 \times m}$, then $z=\sum_{i=1}^{m} a_{i} e_{i}$, where $a_{i} \in D$, and thus, $d z=0 \Rightarrow d a_{i}=0, i=1, \ldots, m$, because $\left\{e_{i}\right\}_{1 \leq i \leq m}$ is a basis, which proves that $a_{i}=0, i=1, \ldots, m$, i.e., $z=0$, since $0 \neq d \in D$ and $D$ is an integral domain).

1. $\Leftarrow$ Let us consider the following free resolution of $\widetilde{N}$ :

$$
0 \longleftarrow \tilde{N} \longleftarrow D^{1 \times q} \stackrel{\cdot \theta(R)}{\longleftarrow} D^{1 \times p} \stackrel{\cdot \theta\left(R_{-1}\right)}{\longleftarrow} D^{1 \times m} .
$$

Using the fact that $t(M) \cong \operatorname{ext}_{D}^{1}(N, D)=0$, we have the following exact sequence:

$$
D^{1 \times q} \stackrel{. R}{\longrightarrow} D^{1 \times p} \stackrel{. R_{-1}}{\longrightarrow} D^{1 \times m} .
$$

Therefore, we have $M=D^{1 \times p} / D^{1 \times q} R=$ coker.$R \cong \mathrm{im} \cdot R_{-1} \cong D^{1 \times p} R_{-1}$, i.e., $R y=0$ is parametrized by means of $R_{-1}$.

2. Using the fact that $M$ is defined by a full row rank matrix, we have the exact sequence:

$$
0 \longrightarrow D^{1 \times q} \stackrel{. R}{\longrightarrow} D^{1 \times p} \longrightarrow M \longrightarrow 0 .
$$

Dualizing this exact sequence, we obtain the following exact sequence

$$
0 \longleftarrow N \longleftarrow D^{q} \stackrel{R .}{\longleftarrow} D^{p} \longleftarrow M^{*} \longleftarrow 0,
$$

where we easily check that $N=\operatorname{ext}_{D}^{1}(M, D)$.

$a \Rightarrow b$. Let us suppose that $M$ is a projective left $D$-module. Therefore, (28) is a split exact sequence because it ends in a projective left $D$-module (see Proposition 3.8), i.e., there exists $S \in D^{p \times q}$ such that $R S=I_{q}$. 
$b \Rightarrow c$. Let us suppose that there exists $S \in D^{p \times q}$ such that $R S=I_{q}$. Hence, for all $\lambda \in D^{q}$, we have $R \mu=\lambda$ with $\mu=S \lambda \in D^{p}$. Therefore, the $D$-morphism $R .: D^{p} \rightarrow D^{q}$ is surjective, and thus, $N=\operatorname{coker} R .=0$.

$c \Rightarrow a$. Let us suppose that $N=0$. Then, (29) ends in a free $D$-module, and thus, (29) splits (see Proposition 3.8), i.e., there exists $S \in D^{p \times q}$ such that $R S=I_{q}$. Therefore, (28) is also a split exact sequence, and thus, we have $D^{1 \times p} \cong D^{1 \times q} \oplus M$, which proves that $M$ is a projective left $D$-module.

Example 8.9. Let us check whether or not the differential time-delay system defined by (4) is controllable or, equivalently, parametrizable. By Proposition 4.2, we know that (4) is controllable iff the $D_{h}$-module $M=D_{h}^{1 \times 4} / D_{h}^{1 \times 3} R$ is torsion-free, where $R$ is defined by (5). By Theorem 8.3, this is equivalent to check $\operatorname{ext}_{D_{h}}^{1}\left(N, D_{h}\right)=0$, where $N=D_{h}^{1 \times 3} / D_{h}^{1 \times 4} R^{T}$ (see 1 of Example 6.5). Therefore, system (4) is controllable and, using 1 of Corollary 8.8, we deduce that a parametrization of (4) is given by the matrix $R_{-1}$ defined in Example 5.5:

$$
\left\{\begin{array}{l}
\left(\omega^{2} k a \delta_{h}\right) z(t)=x_{1}(t) \\
\left(\omega^{2} \partial+\omega^{2} a\right) z(t)=x_{2}(t) \\
\left(\omega^{2} \partial^{2}+\omega^{2} a \partial\right) z(t)=x_{3}(t) \\
\left(\partial^{3}+(2 \zeta \omega+a) \partial^{2}+\left(\omega^{2}+2 a \zeta \omega\right) \partial+a \omega^{2}\right) z(t)=u(t)
\end{array} \Rightarrow(4)\right.
$$

Finally, the fact that $\operatorname{ext}_{D_{h}}^{2}\left(N, D_{h}\right) \neq 0$ implies that $M=D_{h}^{1 \times 4} / D_{h}^{1 \times 3} R$ is not a projective, and thus, is not a free $D_{h}$-module (see 2 of Theorem 3.6). The obstruction for $M$ to be free is given by (14): the fact that system (14) is not equivalent to $y=0$ means that it is not possible to express $z$ in terms of a $D_{h}$-linear combination of $x_{1}, x_{2}, x_{3}$ and $u$. Therefore, (4) is not a flat differential time-delay system.

\section{$9 \pi$-freeness, minimal parametrizations and flatness}

\subsection{Left-Inverses \& $\pi$-freeness}

In order to check the flatness of a linear control system, we need to know whether or not the associated $D$-module is projective, and thus, by Theorem 8.7, we need to compute $\operatorname{ext}_{D}^{i}(N, D)$ for $i=1, \ldots, \operatorname{rgld} D$. However, if the system is defined by a full row rank matrix, then 2 of Corollary 8.8 gives a more economic way to check projectiveness. We need to check if $R$ admits a right-inverse $S$.

\section{Algorithm 9.1.}

Input: $\quad$ An Ore algebra $D$ satisfying (2) and a matrix $R \in D^{q \times p}$.

Output: A matrix $S \in D^{p \times q}$ satisfying $S R=I_{p}$ if it exists and [ ] otherwise.

$\operatorname{LeFT-\operatorname {InVERSE}}(R)$

Introduce the indeterminates $\lambda_{j}, j=1, \ldots, p$ and $\mu_{i}, i=1, \ldots, q$, over $D$.

$P \leftarrow\left\{\sum_{j=1}^{p} R_{i j} \lambda_{j}-\mu_{i} \mid i=1, \ldots, q\right\}$,

Compute the Gröbner basis $G$ of $P$ in $\bigoplus_{i=1}^{p} D \lambda_{i} \oplus \bigoplus_{i=1}^{q} D \mu_{i}$ w.r.t. an order which eliminates the $\lambda_{i}$ 's.

Remove from $G$ the elements which do not contain any $\lambda_{i}$ and call $G^{\prime}$ this new set.

Write $G^{\prime}$ in the form $Q_{1} \cdot\left(\lambda_{1}: \ldots: \lambda_{p}\right)^{T}-Q_{2} \cdot\left(\mu_{1}: \ldots: \mu_{q}\right)^{T}$.

If $Q_{1}$ is invertible in $D$, then return $S=Q_{1}^{-1} Q_{2} \in D^{p \times q}$, else return [ ].

Now, we can compute a right-inverse $S \in D^{p \times q}$ of $R \in D^{q \times p}\left(R S=I_{q}\right)$, when such an inverse exists, by doing $\operatorname{Right-\operatorname {Inverse}}(R)=\theta(\operatorname{LefT}-\operatorname{Inverse}(\theta(R)))$. Therefore, if $R \in D^{q \times p}$ has full row rank, by 2 of Corollary 8.8, the left $D$-module $M=D^{1 \times p} / D^{1 \times q} R$ is projective iff $\operatorname{Right-\operatorname {InVERSE}}(R) \neq[]$.

Example 9.2. Let us consider again the differential time-delay system defined by (4). Applying Algorithm 9.1 to $\theta(R)=R^{T}$, where $R$ is defined by (5), we are led to the Gröbner basis $G$ defined in Example 5.5. We easily check that $G$ does not contain any relation of the form $\lambda_{i}-\sum_{j=1}^{4} S_{i j} \mu_{j}$, where $S_{i j} \in D_{h}$, for $i=1, \ldots, 3$. Therefore, $M=D_{h}^{1 \times 4} / D_{h}^{1 \times 3} R$ is not a projective $D_{h}$-module, and thus, (4) is not a flat system [20] (see also Example 8.9). 
If $D=k\left[x_{1}, \ldots, x_{n}\right]$ is a commutative polynomial ring over a field $k$, then we can study the obstructions for a system to be free (i.e., projective by the Quillen-Suslin theorem stated in Theorem 3.6). In this case, they are given by polynomials containing a certain number of variables $x_{i}$ which depends on the properties of the corresponding $D$-module $M$.

Definition 9.3. Let $D=k\left[x_{1}, \ldots, x_{n}\right], R \in D^{q \times p}$ be a full row rank matrix and let us define the $D$-module $M=D^{1 \times p} / D^{1 \times q} R$. Then, let us define:

$$
i(M)=\min _{i \geq 1}\left\{i-1 \mid \operatorname{ext}_{D}^{i}(N, D) \neq 0\right\} \in\{0, \ldots, n-1,+\infty\} .
$$

$i(M)$ is called the torsion-free degree of $M$.

Let us notice that the torsion-free degree of the module $M=D^{1 \times p} / D^{1 \times q} R$ over $D=k\left[x_{1}, \ldots, x_{n}\right]$ measures how far $M$ is from projectivity. In particular, $i(M)=0$ means that $M$ has some torsion elements, whereas $i(M)=1$ means that $M$ is a torsion-free but not reflexive $D$-module, .., and $i(M)=+\infty$ means that for all $i \geq 1$, we have $\operatorname{ext}_{D}^{i}(N, D)=0$, i.e., $M$ is a projective $D$-module.

Proposition 9.4. [31] Let $D=k\left[x_{1}, \ldots, x_{n}\right], R \in D^{q \times p}$ be a full row rank matrix and the $D$-modules $M=D^{1 \times p} / D^{1 \times q} R$ and $N=D^{1 \times q} / D^{1 \times p} R^{T}$. For every partition of $X=\left\{x_{1}, \ldots, x_{n}\right\}$ into two disjoint subsets $X_{1}$ and $X_{2}$ with respectively $n-i(M)$ elements and $i(M)$ elements, there exists

$$
\begin{cases}\pi_{n-i(M)} \in k\left[X_{1}\right], & \text { if } 0 \leq i(M) \leq n-1, \\ \pi_{n-i(M)} \in k, & \text { if } i(M)=+\infty,\end{cases}
$$

such that the module over $D_{\pi_{n-i(M)}}=\left\{d / a \mid d \in D, a=\pi_{n-i(M)}^{m}, m \in \mathbb{Z}_{\geq 0}\right\}$

$$
D_{\pi_{n-i(M)}} \otimes_{D} M=\left\{m / a \mid m \in M, a=\pi_{n-i(M)}^{m}, m \in \mathbb{Z}_{\geq 0}\right\}
$$

is free. Moreover, there exist $R_{-1} \in D^{p \times(p-q)}, T \in D^{p \times q}, T_{-1} \in D^{(p-q) \times p}$ and $\nu \in \mathbb{Z}_{\geq 0}$ such that:

$$
\left(T: R_{-1}\right)\left(\begin{array}{c}
R \\
T_{-1}
\end{array}\right)=\pi_{n-i(M)}^{\nu} I_{p}, \quad\left(\begin{array}{c}
R \\
T_{-1}
\end{array}\right)\left(T: R_{-1}\right)=\pi_{n-i(M)}^{\nu} I_{p} .
$$

Proof. If $i(M)=+\infty$, then, using 2 of Proposition 5.6, we have:

$$
\operatorname{ext}_{D}^{i}(N, D)=0, \quad i=1, \ldots, \operatorname{gld} D=n .
$$

So, by Proposition 8.7, $M$ is a projective $D$-module, and thus, by 2 of Theorem $3.6, M$ is a free $D$-module. Hence, we can choose $\pi_{n-\infty}=1 \in k \backslash 0$ and we have $D_{\pi_{n-\infty}}=D$. Using the fact that $R$ has full row rank, then we have the following exact sequence:

$$
0 \longrightarrow D^{1 \times q} \stackrel{. R}{\longrightarrow} D^{1 \times p} \longrightarrow M \longrightarrow 0 .
$$

We apply the functor $K \otimes_{D} \cdot$ to the exact sequence (32), where $K=Q(D)$ is the quotient field of $D$. Using the fact that $K$ is a flat $D$-module, then we obtain the exact sequence

$$
0 \longrightarrow K^{1 \times q} \stackrel{. R}{\longrightarrow} K^{1 \times p} \longrightarrow K \otimes M \longrightarrow 0,
$$

which shows that $\operatorname{rank}_{D}(M)=\operatorname{dim}_{K}\left(K \otimes_{D} M\right)=\operatorname{dim}_{K} K^{1 \times p}-\operatorname{dim}_{K} K^{1 \times q}=p-q$. Hence, since $M$ is free, therefore isomorphic to $D^{p-q}$, we obtain the split exact sequence

$$
0 \longrightarrow D^{1 \times q} \underset{\stackrel{. R}{\rightleftarrows}}{\stackrel{. R}{\longrightarrow}} D^{1 \times p} \underset{\stackrel{. T_{-1}}{\stackrel{. R_{-1}}{\longrightarrow}}}{\stackrel{.}{\leftrightarrows}} D^{1 \times(p-q)} \longrightarrow 0
$$

and thus, identities (31).

Now, let us suppose that we have $0 \leq i(M) \leq n-1$. Then, we have:

$$
\operatorname{ext}_{D}^{j}(N, D)=0, \quad 0 \leq j \leq i(M) \text {. }
$$


Let us consider the multiplicatively closed subset $S=k\left[X_{1}\right] \backslash\{0\}$ of $D$ and $S^{-1} D=k\left(X_{1}\right)\left[X_{2}\right]$. By 2 of Proposition 5.6, we have gld $S^{-1} D=i(M)$, and thus, for the $S^{-1} D$-module $S^{-1} D \otimes_{D} N$, we have:

$$
\forall j \geq i(M)+1, \quad \operatorname{ext}_{S^{-1} D}^{j}\left(S^{-1} D \otimes_{D} N, S^{-1} D\right)=0 .
$$

Then, by Lemma 3.9, we conclude that:

$$
\forall j \geq i(M)+1, \quad S^{-1} D \otimes_{D} \operatorname{ext}_{D}^{j}(N, D) \cong \operatorname{ext}_{S^{-1} D}^{j}\left(S^{-1} D \otimes_{D} N, S^{-1} D\right)=0,
$$

i.e., $S^{-1} D \otimes_{D} \operatorname{ext}_{D}^{j}(N, D)=0$, for all $j \geq i(M)+1$.

Let us consider $i(M)+1 \leq j \leq n$. One can prove that a non-zero $\operatorname{ext}_{D}^{j}(N, D)$ is a torsion $D$-module for $j \geq 1$ (see $[25,29]$ for more details). So, if $\operatorname{ext}_{D}^{j}(N, D) \neq 0$, then:

$$
\operatorname{ann}\left(\operatorname{ext}_{D}^{j}(N, D)\right)=\left\{P \in D \mid \forall m \in \operatorname{ext}_{D}^{j}(N, D), P m=0\right\} \neq 0 .
$$

If $i_{S^{-1} D}: \operatorname{ext}_{D}^{j}(N, D) \rightarrow S^{-1} D \otimes \operatorname{ext}_{D}^{j}(N, D)$ denotes the canonical map (see (20) for more details), then, for $0 \neq z \in \operatorname{ext}_{D}^{j}(N, D)$, we have $i_{S^{-1}} D(z)=0$ iff $\{P \in D \mid P z=0\} \cap S \neq \emptyset$. Therefore, using the fact that $S^{-1} D \otimes \operatorname{ext}_{D}^{j}(N, D)=0$, we obtain:

$$
\operatorname{ann}\left(\operatorname{ext}_{D}^{j}(N, D)\right) \cap\left(k\left[X_{1}\right] \backslash\{0\}\right) \neq \emptyset .
$$

For $i(M)+1 \leq j \leq n$, if $\operatorname{ext}_{D}^{j}(N, D) \neq 0$, we take any $p_{j} \in \operatorname{ann}\left(\operatorname{ext}_{D}^{j}(N, D)\right) \cap\left(k\left[X_{1}\right] \backslash\{0\}\right)$, else, we take $p_{j}=1$. Let us denote by $\pi_{n-i(M)}$ the product of the $p_{j}$ for $i(M)+1 \leq j \leq n$. By construction, we have $\pi_{n-i(M)} \in k\left[X_{1}\right]$ and:

$$
\pi_{n-i(M)} \operatorname{ext}_{D}^{j}(N, D)=0, \quad 0 \leq j \leq n .
$$

If we denote by $S_{\pi_{n-i(M)}}=\left\{1, \pi_{n-i(M)}, \pi_{n-i(M)}^{2}, \ldots\right\}$ the multiplicatively closed subset of $D$ defined by $\pi_{n-i(M)}$ and $D_{\pi_{n-i(M)}} \triangleq S_{\pi_{n-i(M)}^{-1}} D=\left\{d / a \mid d \in D, a=\left(\pi_{n-i(M)}\right)^{m}, m \in \mathbb{Z}_{\geq 0}\right\}$, then, using Lemma 3.9, from $D_{\pi_{n-i(M)}} \otimes_{D} \operatorname{ext}_{D}^{j}(N, D)=0,0 \leq j \leq n$, we conclude that:

$$
\operatorname{ext}_{D_{\pi_{n-i(M)}}^{j}}^{j}\left(D_{\pi_{n-i(M)}} \otimes_{D} N, D_{\pi_{n-i(M)}}\right)=0, \quad 0 \leq j \leq n
$$

Since the transposed module of $M^{\prime} \triangleq D_{\pi_{n-i(M)}} \otimes_{D} M$ is $D_{\pi_{n-i(M)}} \otimes_{D} N$, by Proposition 8.7, it follows that $M^{\prime}$ is a projective $D_{\pi_{n-i(M)}}$-module, and thus, by 2 of Theorem 3.6, $M^{\prime}$ is a free $D_{\pi_{n-i(M)}}$-module.

Now, using the fact that $M^{\prime}$ is a free $D_{\pi_{n-i(M)}}$-module of rank $p-q$, there exist $R_{-1}^{\prime} \in D_{\pi_{n-i(M)}}^{p \times(p-q)}$, $T^{\prime} \in D_{\pi_{n-i(M)}}^{p \times q}$ and $T_{-1}^{\prime} \in D_{\pi_{n-i(M)}}^{(p-q) \times p}$ such that we have the following split exact sequence

$$
0 \longrightarrow D_{\pi_{n-i(M)}^{1 \times q}} \underset{\stackrel{. R}{\rightleftarrows}}{\stackrel{. T^{\prime}}{\longleftarrow}} D_{\pi_{n-i(M)}^{1 \times p}}^{\stackrel{. R_{-1}^{\prime}}{\longrightarrow}} D_{\pi_{n-i(M)}}^{1 \times(p-q)} \longrightarrow 0
$$

or, equivalently, the following identities:

$$
\left(T^{\prime}: R_{-1}^{\prime}\right)\left(\begin{array}{c}
R \\
T_{-1}^{\prime}
\end{array}\right)=I_{p}, \quad\left(\begin{array}{c}
R \\
T_{-1}^{\prime}
\end{array}\right)\left(T^{\prime}: R_{-1}^{\prime}\right)=I_{p}
$$

Collecting common denominators yields matrices $R_{-1} \in D^{p \times(p-q)}, T \in D^{p \times q}, T_{-1} \in D^{(p-q) \times p}$ and $\nu \in \mathbb{Z}_{\geq 0}$ as asserted in the theorem.

In order to compute such a polynomial $\pi_{n-i(M)}$, we can follow $\pi$-POLYNOMIAL [31].

\section{Algorithm 9.5.}

Input: A commutative polynomial ring $D=k\left[x_{1}, \ldots, x_{n}\right]$, where $k$ is a field, a left $D$-module $M=D^{1 \times p} / D^{1 \times q} R, i(M)$ the torsion-free degree of $M$, a partition of $X=\left\{x_{1}, \ldots, x_{n}\right\}$ into disjoint subsets $X_{1}$ and $X_{2}$ with respectively $n-i(M)$ elements and $i(M)$ elements.

Output: An ideal $J$ of $k\left[X_{1}\right]$ such that any element $\pi \in J$ satisfies that $D_{\pi} \otimes_{D} M$ is a free $D_{\pi}$-module. 
$\pi$-POLYNOMIAL $\left(R, X_{1}\right)$

$$
\begin{aligned}
& R_{1} \leftarrow R . \\
& \text { for } i=i(M)+1, \ldots, n \text { do } \\
& \quad L_{i}=\left[L_{i 0}, L_{i 1}, L_{i 2}\right] \leftarrow \operatorname{ExT} 1\left(R_{i}\right) . \\
& \quad \text { Introduce the indeterminates } \mu_{j}, j=1, \ldots, \operatorname{rowdim}\left(L_{i 1}\right) \text { over } D . \\
& \quad P_{i j} \leftarrow\left\{d \in D \mid d \cdot \mu_{j} \in L_{i 0}\right\}, j=1, \ldots, \operatorname{rowdim}\left(L_{i 1}\right) . \\
& \quad R_{i+1} \leftarrow \operatorname{SYZYGIES}\left(R_{i}\right) . \\
& \text { endfor } \\
& I \leftarrow \bigcap_{i, j}\left\langle P_{i j}\right\rangle . \\
& J \leftarrow I \cap k\left[X_{1}\right] .
\end{aligned}
$$

In $\pi$-POLYNOMIAL, the set $\left\{d \in D \mid d \cdot \mu_{j} \in L_{i 0}\right\}$ is obtained in the step Quotient of $\operatorname{ExT} 1\left(R_{i}\right)$. See Remark 7.6 for more details. Moreover, in the last step of $\pi$-POLYNOMIAL, the intersection of ideals of Ore algebras can be computed by means of elimination algorithms (see [2] and Example 2.15).

Example 9.6. In Examples 8.9 and 9.2, we proved that the linear differential time-delay system defined by (4) is not flat, i.e., the associated $D_{h}$-module $M=D_{h}^{1 \times 4} / D_{h}^{1 \times 3} R$ is not free. Let us find a polynomial $\pi \in \mathbb{R}(a, k, \zeta, \omega)\left[\delta_{h} ; \sigma_{2}, \delta_{2}\right]$ such that the $\left(D_{h}\right)_{\pi}$-module $\left(D_{h}\right)_{\pi} \otimes_{D_{h}} M$ is free. In Example 7.4, we saw that $\operatorname{ext}_{D_{h}}^{1}\left(N, D_{h}\right)=0$ and $\operatorname{ext}_{D_{h}}^{2}\left(N, D_{h}\right) \neq 0$. Therefore, the torsion-free degree $i(M)$ of $M$ is 1 . Applying Algorithm 9.5 and using (14), we find that:

$$
I=\left(\omega^{2} k a \delta_{h}, \omega^{2} \partial+\omega^{2} a, \omega^{2} \partial^{2}+\omega^{2} a \partial, \partial^{3}+(2 \zeta \omega+a) \partial^{2}+\left(\omega^{2}+2 a \zeta \omega\right)+a \omega^{2}\right) .
$$

Thus, we have $I \cap \mathbb{R}(a, k, \zeta, \omega)\left[\delta_{h} ; \sigma_{2}, \delta_{2}\right]=\left(\delta_{h}\right)$, which shows that (4) is $\delta_{h}$-free [20].

We refer to [31] for an extension of Proposition 9.4 to none full row rank matrices.

\subsection{Minimal parametrizations and flatness}

The next theorem generalizes a result of [26] obtained for systems of partial differential equations. We assume that $D$ is a left/right noetherian integral domain.

Theorem 9.7. [30] Let $M$ be a torsion-free left D-module defined by the finite presentation (15). Then, there always exists a left $D$-morphism $d_{0}^{\prime}: F_{0} \longrightarrow F_{-1}^{\prime}$, where $F_{-1}^{\prime}$ is a finitely generated free left D-module, such that we have the following exact sequence

$$
F_{1} \stackrel{d_{1}}{\longrightarrow} F_{0} \stackrel{d_{0}^{\prime}}{\longrightarrow} F_{-1}^{\prime}
$$

and $M_{-1}^{\prime}=$ coker $d_{0}^{\prime}$ is either 0 or a torsion left D-module. Such a morphism $d_{0}^{\prime}$ is then called a minimal parametrization of $M$.

Proof. Let us consider the beginning of a free resolution of the transposed right $D$-module $N$ of $M$, i.e., we have the following exact sequence

$$
0 \longleftarrow N \longleftarrow F_{1}^{*} \stackrel{d_{1}^{*}}{\longleftarrow} F_{0}^{*} \stackrel{d_{0}^{*}}{\longleftarrow} F_{-1}^{*} \longleftarrow L \longleftarrow 0
$$

where $F_{-1}^{*}$ is a finitely generated free right $D$-module and $L=\operatorname{ker} d_{0}^{*}$.

If we have $L=0$, dualizing the previous exact sequence and using the fact that $M$ is a torsion-free left $D$-module, and thus, $\operatorname{ext}_{D}^{1}(N, D)=0$ by Theorem 8.3 , then the following complex

$$
F_{1} \stackrel{d_{1}}{\longrightarrow} F_{0} \stackrel{d_{0}}{\longrightarrow} F_{-1} \longrightarrow 0
$$

is exact in $F_{0}$. Then, we have $\operatorname{ext}_{D}^{2}(N, D)=$ coker $d_{0}$. Hence, $\operatorname{ext}_{D}^{2}(N, D)$ is either 0 if $M$ is a reflexive left $D$-module (and thus, $M \cong \operatorname{im~} d_{0}=F_{-1}$ is a free left $D$-module) or, by Corollary 8.6, $\operatorname{ext}_{D}^{2}(N, D)$ is a non-zero torsion left $D$-module because we have $\operatorname{hom}_{D}\left(\operatorname{ext}_{D}^{2}(N, D), D\right)=0$. Hence, $d_{0}^{\prime}=d_{0}$ and $F_{0}^{\prime}=F_{0}$ satisfy the theorem and $d_{0}$ is a minimal parametrization of $M$.

Now, let us suppose that $L \neq 0$. Then, we have the following exact sequence

$$
0 \longleftarrow \operatorname{im} d_{0}^{*} \longleftarrow F_{-1}^{*} \longleftarrow L \longleftarrow 0,
$$

from which we deduce that:

$$
\operatorname{rank}_{D} \operatorname{im} d_{0}^{*}=\operatorname{rank}_{D} F_{-1}^{*}-\operatorname{rank}_{D} L \geq 1
$$


Indeed, if $\operatorname{rank}_{D} \operatorname{im} d_{0}^{*}=\operatorname{dim}_{K}\left(K \otimes_{D} \operatorname{im} d_{0}^{*}\right)=0$, then $K \otimes_{D} \operatorname{im} d_{0}^{*}=0$, i.e., im $d_{0}^{*}$ is a torsion right $D$-module. But, $\operatorname{im} d_{0}^{*} \subseteq F_{0}^{*}$ and $F_{0}^{*}$ is a free, and thus, torsion-free right $D$-module. Hence, im $d_{0}^{*}=0$, and thus, $F_{-1}^{*}=0$ which contradicts the hypothesis that $L \neq 0$. Hence, we have $l^{\prime} \triangleq \operatorname{rank}_{D} \operatorname{im} d_{0}^{*} \geq 1$.

From the matrix $R_{0}$ which corresponds to $d_{0}^{*}$ in the canonical basis of $F_{-1}^{*}$ and $F_{0}^{*}$, let us extract a $\left(\operatorname{rank}_{D} F_{0}^{*} \times l^{\prime}\right)$-submatrix $R_{0}^{\prime}$ composed by $D$-linear independent columns of $R_{0}$, which, in turn, defines a right $D$-morphism $d_{0}^{\prime *}: F_{-1}^{\prime *} \longrightarrow F_{0}^{*}$ in the canonical basis, where $F_{-1}^{\prime *}$ is a free right $D$ module of rank $l^{\prime}$. Using the fact that $R_{0}^{\prime}$ has full column rank, then $d_{0}^{\prime *}$ is injective. Thus, we have the following commutative exact diagram

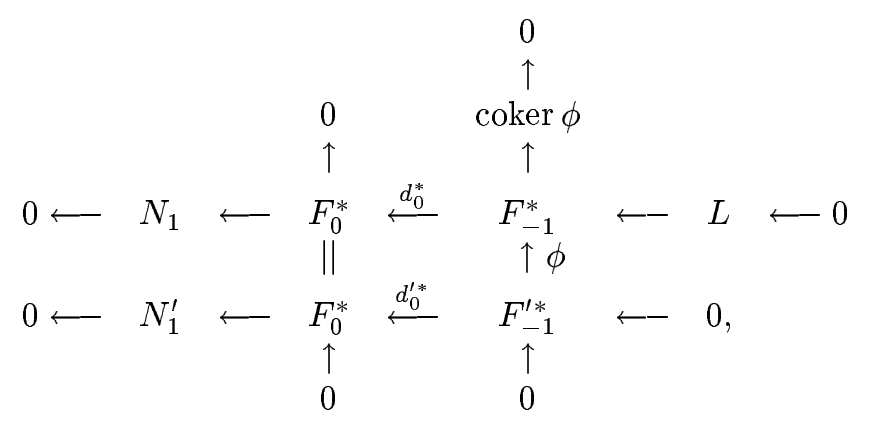

where $d_{0}^{\prime *}=d_{0}^{*} \circ \phi, N_{1}=$ coker $d_{-1}^{*}$ and $N_{1}^{\prime}=\operatorname{coker} d_{-1}^{\prime *}$. Using the snake lemma [4, 33], we obtain the following exact sequence $0 \longleftarrow N_{1} \stackrel{\psi}{\longleftarrow} N_{1}^{\prime} \longleftarrow$ coker $\phi \longleftarrow L \longleftarrow 0$. Using (33), we have:

$$
\begin{aligned}
\operatorname{rank}_{D} N_{1}-\operatorname{rank}_{D} N_{1}^{\prime} & =\operatorname{rank}_{D} L-\operatorname{rank}_{D} \operatorname{coker} \phi=\operatorname{rank}_{D} L-\operatorname{rank}_{D} F_{-1}^{*}+\operatorname{rank}_{D} F_{-1}^{\prime *} \\
& =-\operatorname{rank}_{D} \operatorname{im} d_{0}^{*}+\operatorname{rank}_{D} F_{-1}^{\prime *}=0 .
\end{aligned}
$$

Therefore, we have $\operatorname{rank}_{D} N_{1}=\operatorname{rank}_{D} N_{1}^{\prime}$, and thus, $\operatorname{rank}_{D}$ ker $\psi=0$ because we have the exact sequence $0 \longleftarrow N_{1} \stackrel{\psi}{\longleftarrow} N_{1}^{\prime} \longleftarrow \operatorname{ker} \psi \longleftarrow 0$. Hence, $\operatorname{ker} \psi$ is a torsion right $D$-module. This fact implies that $\operatorname{hom}_{D}(\operatorname{ker} \psi, D)=0$ and, dualizing the previous exact sequence, we have $N_{1}^{*} \triangleq$ $\operatorname{hom}_{D}\left(N_{1}, D\right)=N_{1}^{\prime *} \triangleq \operatorname{hom}_{D}\left(N_{1}^{\prime}, D\right)$. Dualizing the commutative exact diagram (34), we obtain the following commutative exact diagram:

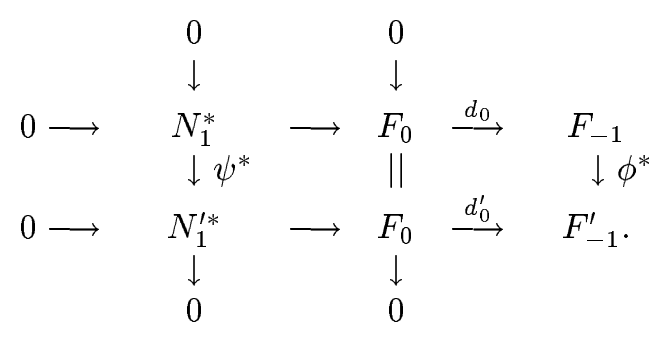

Therefore, we have ker $d_{0}=N_{1}^{*}$ and ker $d_{0}^{\prime}=N_{1}^{\prime *}$. Hence, the defect of exactness of the following complex

$$
F_{1} \stackrel{d_{1}}{\longrightarrow} F_{0} \stackrel{d_{0}^{\prime}}{\longrightarrow} F_{-1}^{\prime}
$$

at $F_{0}$ is defined by $H\left(F_{0}\right)=\operatorname{ker} d_{0}^{\prime} / \operatorname{im} d_{1}=\operatorname{ker} d_{0}^{\prime} / \operatorname{ker} d_{0}$, because $\operatorname{ker} d_{0}=\operatorname{im} d_{1}$ as we have $\operatorname{ext}_{D}^{1}(N, D)=0$. Finally, we obtain that $H\left(F_{0}\right)=N_{1}^{*} / N_{1}^{\prime *}=0$ because we have $N_{1}^{*}=N_{1}^{\prime *}$. Thus, (35) is an exact sequence, and thus, $d_{0}^{\prime}$ is a parametrization of $M$. Finally, we have $\operatorname{hom}_{D}\left(\operatorname{coker} d_{0}^{\prime}, D\right)=$ ker $d_{0}^{\prime *}=0$, and thus, by Corollary 8.6, coker $d_{0}^{\prime}$ is a torsion left $D$-module, which proves the result.

\section{Algorithm 9.8.}

Input: $\quad$ An Ore algebra $D$ satisfying (2) with an involution $\theta$, a torsion-free left $D$-module $M=D^{1 \times p} / D^{1 \times q} R$.

Output: A minimal parametrization $R_{-1}^{\prime} \in D^{p \times m}$ of $M$.

\section{Minimal-Parametrization $(R)$}

$L \leftarrow \operatorname{SYZYGIES}(\theta(R))$.

$m \leftarrow \operatorname{rank}_{\mathrm{D}}(L)$.

Select $m D$-linearly independent rows of $L$ and form a $p \times m$-matrix $L^{\prime}$ with them.

$R_{-1}^{\prime} \leftarrow \theta\left(L^{\prime}\right)$. 
Example 9.9. Let us consider the first set of Maxwell equations [15], namely

$$
\left\{\begin{array}{l}
\frac{\partial \vec{B}}{\partial t}+\vec{\nabla} \wedge \vec{E}=0 \\
\vec{\nabla} \cdot \vec{B}=0
\end{array}\right.
$$

where $\vec{B}$ (resp. $\vec{E}$ ) denotes the magnetic (resp. electric) field. In electromagnetism, it is well-known that (36) is parametrized by

$$
\left\{\begin{array}{l}
\vec{\nabla} \wedge \vec{A}=\vec{B} \\
-\nabla V-\frac{\partial \vec{A}}{\partial t}=\vec{E}
\end{array}\right.
$$

where $(\vec{A}, V)$ is called quadri-potential [15]. In terms of module theory, this result means that the $D$ module $M=D^{1 \times 6} / D^{1 \times 4} R$ is torsion-free, where $D=A_{4}$ denotes the Weyl algebra (see Example 2.3), $x_{4}=t$ the time variable, and $R$ is the matrix of differential operators defining (36), namely:

$$
R=\left(\begin{array}{cccccc}
\partial_{4} & 0 & 0 & 0 & -\partial_{3} & \partial_{2} \\
0 & \partial_{4} & 0 & \partial_{3} & 0 & -\partial_{1} \\
0 & 0 & \partial_{4} & -\partial_{2} & \partial_{1} & 0 \\
\partial_{1} & \partial_{2} & \partial_{3} & 0 & 0 & 0
\end{array}\right) \in D^{4 \times 6}
$$

We easily check that a free resolution of the $D$-module $M$ is defined by

$$
0 \longrightarrow D \stackrel{. R_{2}}{\longrightarrow} D^{1 \times 4} \stackrel{\cdot R}{\longrightarrow} D^{1 \times 6} \longrightarrow M \longrightarrow 0,
$$

where $R_{2}=\left(\partial_{1}: \partial_{2}: \partial_{3}:-\partial_{4}\right) \in D^{1 \times 4}$. Therefore, we obtain $\operatorname{rank}_{D}(M)=6-4+1=3$ or, in other words, system $(36)$ is defined by $4-1=3 D$-linearly independent equations in 6 unknowns $(\vec{B}, \vec{E})$, and thus, its solutions depend on 3 arbitrary functions of $\left(x_{1}, x_{2}, x_{3}, x_{4}\right)$. But, the parametrization (37) of the torsion-free $D$-module $M$ depends on 4 arbitrary potentials, namely $(\vec{A}, V)$. Hence, by Theorem 9.7, we know that system (36) admits some parametrizations with only 3 arbitrary potentials. Let us compute such parametrizations following Algorithm 9.8. Using Algorithm 5.3, the $D$-module $N=D^{1 \times 4} / D^{1 \times 6} R^{T}$ admits the following free resolution

$$
0 \longleftarrow N \longleftarrow D^{1 \times 4} \stackrel{. R^{T}}{\longleftarrow} D^{1 \times 6} \stackrel{. R_{-1}^{T}}{\longleftarrow} D^{1 \times 4} \stackrel{. R_{-2}^{T}}{\longleftarrow} D \longleftarrow 0
$$

where:

$$
R_{-1}^{T}=\left(\begin{array}{cccccc}
0 & -\partial_{3} & \partial_{2} & \partial_{4} & 0 & 0 \\
\partial_{3} & 0 & -\partial_{1} & 0 & \partial_{4} & 0 \\
-\partial_{2} & \partial_{1} & 0 & 0 & 0 & \partial_{4} \\
0 & 0 & 0 & -\partial_{1} & -\partial_{2} & -\partial_{3}
\end{array}\right), \quad R_{-2}^{T}=\left(\partial_{1}: \partial_{2}: \partial_{3}: \partial_{4}\right)
$$

Let us point out that

$$
R_{-1}^{T}\left(\begin{array}{c}
\vec{H} \\
-\vec{D}
\end{array}\right)=\left(\begin{array}{c}
\vec{\jmath} \\
\rho
\end{array}\right) \Leftrightarrow\left\{\begin{array}{l}
\vec{\nabla} \wedge \vec{H}-\frac{\partial \vec{D}}{\partial t}=\vec{\jmath} \\
\vec{\nabla} \cdot \vec{D}=\rho,
\end{array}\right.
$$

is exactly the second set of Maxwell equations, where $\vec{\jmath}$ denotes the density of current, $\rho$ the density of electric charge, $\vec{H}$ the magnetic induction and $\vec{D}$ the electric induction. Then, the conservation of current is given by:

$$
R_{-2}^{T}\left(\begin{array}{c}
\vec{\jmath} \\
\rho
\end{array}\right)=0 \quad \Leftrightarrow \quad \frac{\partial \rho}{\partial t}+\vec{\nabla} \cdot \vec{\jmath}=0 .
$$

If we denote by $S\left(R^{T}\right)$ the syzygy module of $R^{T}$, from the free resolution (39) of $N$, we obtain that $\operatorname{rank}_{D} S\left(R^{T}\right)=\operatorname{rank}_{D} D^{1 \times 4} R_{-1}^{T}=4-1=3$. Hence, if we select 3 different rows of $R_{-1}^{T}$ and transpose the corresponding matrix, we obtain a parametrization of (36) with only 3 potentials. Hence, we have the following 4 minimal parametrizations of (36) with only 3 arbitrary potentials $\vec{\xi}=\left(\xi_{1}: \xi_{2}: \xi_{3}\right)^{T}$ :

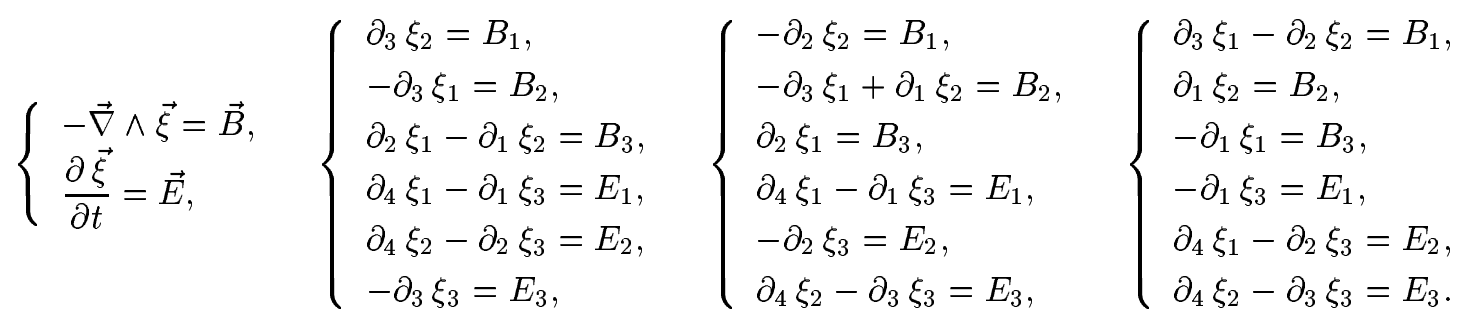

$\mathrm{RR} \mathrm{n}^{\circ} 5181$ 
To finish, let us give a new result which will allow us to compute some bases of a $\pi$-free linear control system.

Proposition 9.10. Let $D=k\left[x_{1}, \ldots, x_{n}\right]$ be a commutative polynomial ring over a field $k$ and $M=$ $D^{1 \times p} / D^{1 \times q} R$ be a torsion-free $D$-module. By Theorem 9.7, there exists a minimal parametrization $R_{-1} \in D^{p \times m}$ of $M$, i.e., a parametrization $R_{-1}$ of $M$ such that $L=D^{1 \times m} / D^{1 \times p} R_{-1}$ is a torsion $D$-module. Then, for all $0 \neq \pi \in \operatorname{ann}(L)=\{P \in D \mid \forall l \in L: P l=0\}$, the $D_{\pi}$-module $D_{\pi} \otimes_{D} M$ is free. In particular, there exists $S_{-1} \in D_{\pi}^{m \times p}$ such that $S_{-1} R_{-1}=I_{m}$ and, with the notations of Section 3, $z=S_{-1} y$ is a basis of the free $D_{\pi}$-module $D_{\pi} \otimes_{D} M$ satisfying $R y=0 \Leftrightarrow y=R_{-1} z$.

Proof. By hypothesis, we have the following exact sequence:

$$
D^{1 \times q} \stackrel{. R}{\longrightarrow} D^{1 \times p} \stackrel{. R_{-1}}{\longrightarrow} D^{1 \times m} \longrightarrow L \longrightarrow 0 .
$$

Moreover, the fact that the $D$-module $L$ is torsion implies that $\operatorname{ann}(L) \neq 0$. If $0 \neq \pi \in \operatorname{ann}(L)$, then, using the fact that $D_{\pi}$ is a flat $D$-module [33], then, by applying the right exact functor $D_{\pi} \otimes_{D} \cdot$ to the exact sequence (40), we obtain the following exact sequence:

$$
D_{\pi}^{1 \times q} \stackrel{. R}{\longrightarrow} D_{\pi}^{1 \times p} \stackrel{\cdot R_{-1}}{\longrightarrow} D_{\pi}^{1 \times m} \longrightarrow D_{\pi} \otimes_{D} L \longrightarrow 0 .
$$

But, by definition of $\pi$, we have $D_{\pi} \otimes_{D} L=0$. Thus, we have $D_{\pi} \otimes_{D} M=D_{\pi}^{1 \times p} / D_{\pi}^{1 \times q} R \cong D_{\pi}^{1 \times m}$, i.e., the $D_{\pi}$-module $D_{\pi} \otimes_{D} M$ is free of rank $m$. Moreover, by Proposition 3.8, the exact sequence (41) splits, and thus, there exists $S_{-1} \in D_{\pi}^{m \times p}$ such that $S_{-1} R_{-1}=I_{m}$. Finally, in terms of equations, in the $D_{\pi}$-module $D_{\pi} \otimes_{D} M$, we have $R y=0 \Leftrightarrow y=R_{-1} z$ and $z=\left(S_{-1} R_{-1}\right) z=S_{-1} y$.

From Proposition 9.10, we easily obtain the following effective algorithm: using Algorithm 9.8, we first compute a minimal parametrization $R_{-1}$ of the torsion-free $D$-module $M$ and then compute $\operatorname{ann}\left(D^{1 \times m} / D^{1 \times p} R_{-1}\right)$. After choosing $0 \neq \pi \in \operatorname{ann}(L)$, using Algorithm 9.1 over $D_{\pi}$, we finally compute a left-inverse $S_{-1}$ of $R_{-1}$, which gives the basis $z=S_{-1} y$ of the $D_{\pi}$-module $D_{\pi} \otimes_{D} M$.

Example 9.11. In Example 8.9, we proved that (30) is a parametrization of system (4). We easily check that (30) is also a minimal parametrization of system (4) as the $D_{h}$-module $L=D_{h} / D_{h}^{1 \times 4} R_{-1}=$ $\operatorname{ext}_{D_{h}}^{2}\left(N, D_{h}\right)$ is torsion. From the definition (14) of $\operatorname{ext}_{D_{h}}^{2}\left(N, D_{h}\right)$, we obtain $\operatorname{ann}(L)=\left(\delta_{h}, \partial+a\right)$. Hence, if we choose $\pi=\delta_{h}$, then we easily check that $S_{-1}=\left(\pi^{-1} /\left(\omega^{2} k a\right): 0: 0: 0\right) \in D_{\pi}^{1 \times 4}$ is a left-inverse of the parametrization $R_{-1}$ defined in Example 5.5. Hence, up to a constant, we obtain that $z=\pi^{-1} x_{1}$, and thus $x_{1}$, are bases (resp. flat outputs) of the $D_{\pi}$-module $D_{\pi} \otimes_{D} M$.

\section{Conclusion}

We hope that we have convinced the reader that the simultaneous use of module theory, homological algebra, effective algebra and computational methods allows us to study effectively the structural properties of linear multidimensional systems (e.g., systems of ODEs, systems of PDEs, (differential) time-delay systems, discrete systems, convolutional codes with constant or variables coefficients). In particular, in this unified mathematical framework, we presented effective algorithms which check controllability/flatness/ $\pi$-freeness... and compute the parametrizations/autonomous elements/flat outputs $/ \pi$-polynomials. Certain of these problems were still open for some classes of linear multidimensional systems [36, 37] and, in particular, for linear differential time-delay systems [11, 20].

The Maple package OreModules, based on Mgfun [6], as well as Maple worksheets containing the explicit examples of the appendix are available at http://wwwb.math.rwth-aachen.de/OreModules. We hope that OreModules will become in the future a platform for the implementation of different algorithms obtained in the literature of multidimensional linear systems (see e.g., $[10,13,23,25,26$, $27,30,32,36,37]$ and the references therein).

\section{A Appendix: Examples}

In this appendix, we give some Maple worksheets which decide controllability, parametrizability, flatness and $\pi$-freeness of some linear time-invariant/time-varying OD systems, differential time-delay systems and systems of PDE with constant or variable coefficients, multidimensional convolutional 
$\operatorname{codes}^{1}$. These results have been obtained using the Maple package OreModules ${ }^{2}$ which is crucially based on the library Mgfun [6] (e.g. Ore algebras and non-commutative Gröbner bases are developed in Mgfun). In these examples, the most time-consuming computation is that of the $\operatorname{ext}_{D}^{i}(N, D)$. We give some timings for these operations. All examples were run on a Pentium II, $450 \mathrm{MHz}$ with $512 \mathrm{MB}$ RAM using Maple 8 (OreModules is available for Maple V release 5, Maple 6, Maple 8, and Maple 9). Finally, we refer the reader to [9] for a complete description of the package OreModules and for more examples using other functions of OreModules.

\section{A.1 Two pendula mounted on a cart}

The first example that we consider is the linearized time-invariant OD system formed by two pendula mounted on a cart. See for more details Examples 5.2.1 and 5.2.12 in [23].

$>$ with(Ore_algebra): with(OreModules):

After loading the required Maple packages, the first step is to define the Ore algebra

$$
D=\mathbb{R}\left(m_{1}, m_{2}, M, L_{1}, L_{2}, g\right)[t][\partial ; \sigma, \delta],
$$

where $\sigma=\operatorname{id}_{\mathbb{R}\left(m_{1}, m_{2}, M, L_{1}, L_{2}, g\right)[t]}$ and $\delta=\frac{d}{d t}$. In what follows, this Ore algebra is denoted by Alg.

$>A l g:=$ DefineOreAlgebra (diff $=[D t, t], \operatorname{polynom}=\{\mathrm{t}\}, \operatorname{comm}=\{\mathrm{m} 1, \mathrm{~m} 2, \mathrm{M}, \mathrm{L} 1, \mathrm{~L} 2, \mathrm{~g}\})$ :

In $A l g$, we need to declare the constants $m_{1}, m_{2}, M, L_{1}, L_{2}$, and $g$ which occur in the system. Then, we define the matrix $R \in D^{3 \times 4}$ which corresponds to the system.

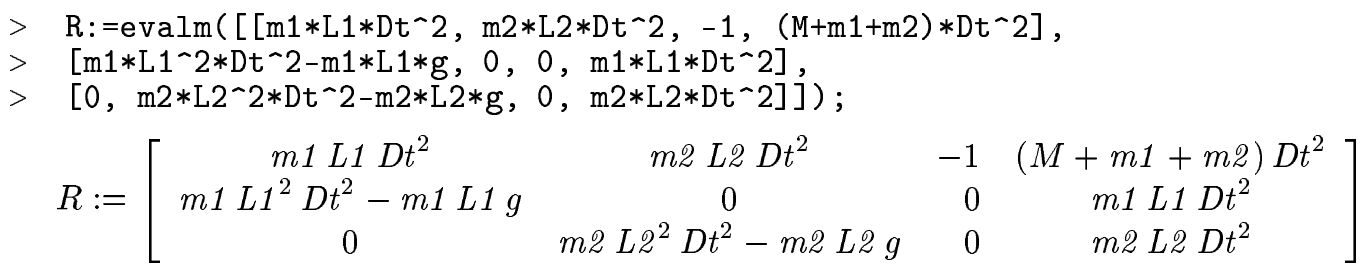

We compute $R_{\text {adj }}=\theta(R) \in D^{4 \times 3}$ using the involution $\theta$ defined in 2 of Example 6.3.

$$
\begin{aligned}
& >R_{-} \text {adj }:=\operatorname{Involution(R,Alg);} \\
& R_{-} a d j:=\left[\begin{array}{ccc}
m 1 L 1 D t^{2} & m 1 L 1^{2} D t^{2}-m 1 L 1 g & 0 \\
m 2 L 2 D t^{2} & 0 & m 2 L 2^{2} D t^{2}-m 2 L 2 g \\
-1 & 0 & 0 \\
D t^{2} M+D t^{2} m 1+D t^{2} m 2 & m 1 L 1 D t^{2} & m 2 L 2 D t^{2}
\end{array}\right]
\end{aligned}
$$

We compute $\operatorname{ext}_{D}^{1}(N, D)$, where $N=D^{1 \times 4} / D^{1 \times 3} R_{\text {adj }}$, using the procedure $\operatorname{ExT} 1\left(R_{\text {adj }}\right)$.

$>$ st:=time(): Ext1:=Exti(R_adj, Alg, 1): time()-st;

The computation of $\operatorname{ext}_{D}^{1}(N, D)$ only takes $1.220 \mathrm{~s}$. Let us notice that all the computations are done generically. In other words, the results are valid for almost all values of the parameters (e.g., outside an algebraic hypersurface).

$>\operatorname{Ext1}[1]$

\footnotetext{
${ }^{1}$ The characteristic $p$ of the ground field $k$ can be chosen in the definition of the Ore algebra using the option "characteristic $=p "$.

${ }^{2}$ OreModules and all examples of this appendix are available on the web page http://wwwb.math.rwth-aachen.de/OreModules.
} 


$$
\left[\begin{array}{lll}
1 & 0 & 0 \\
0 & 1 & 0 \\
0 & 0 & 1
\end{array}\right]
$$

Ext1[1] corresponds to the matrix $L_{0}$ returned by $\operatorname{ExT} 1(R)$ and defined in Algorithm 7.8. In our case, $L_{0}$ is the identity matrix which shows that $\operatorname{ext}_{D}^{1}(N, D)=0$ (see Remark 7.9). Therefore, the system is controllable, and thus, parametrizable (see Proposition 4.2 and Theorem 8.3). The parametrization of the system corresponds to the matrix $L_{2}=\operatorname{Ext1[3]~of~} \operatorname{ExT1}(R)$ (see Algorithm 7.8).

$>\operatorname{map}($ collect, Ext1 [3], Dt);

$$
\begin{aligned}
& {\left[-L 2 D t^{4}+D t^{2} g\right]} \\
& {\left[-D t^{4} L 1+D t^{2} g\right]} \\
& {\left[L 2 D t^{6} L 1 M+(-L 2 g m 1-L 2 g M-g L 1 M-L 1 g m 2) D t^{4}\right.} \\
& \left.\quad+\left(g^{2} M+g^{2} m 1+m 2 g^{2}\right) D t^{2}\right] \\
& {\left[L 2 D t^{4} L 1+(-L 2 g-g L 1) D t^{2}+g^{2}\right]}
\end{aligned}
$$

Thus, we obtain the parametrization $\left(x_{1}: x_{2}: x_{3}: u\right)^{T}=L_{2} z$ of $R\left(x_{1}: x_{2}: x_{3}: u\right)^{T}=0$. Using the fact that $D$ is a principal ideal domain, then, by 1 of Theorem 3.6, we know that the $D$-module $M=D^{1 \times 4} / D^{1 \times 3} R$ is free, and thus, the system defined by $R$ is flat.

$>$ LeftInverse(Ext1[3], Alg);

$$
\left[\begin{array}{llll}
\frac{L 1^{2}}{g^{2}(-L 2+L 1)} & -\frac{L 2^{2}}{g^{2}(-L 2+L 1)} & 0 & -\frac{-L 1+L 2}{g^{2}(-L 2+L 1)}
\end{array}\right]
$$

LeftInverse(Ext1[3], Alg) computes a left-inverse of the parametrization $L_{2}=\operatorname{Ext1}[3]$. We deduce that $z=\left(L_{1}^{2} x_{1}-L_{2}^{2} x_{2}+\left(L_{1}-L_{2}\right) u\right) /\left(g^{2}\left(L_{1}-L_{2}\right)\right)$ is a flat output of the system.

Let us notice that the difference of the pendula lengths $L_{1}-L_{2}$ appears in the denominator of the flat output of the system. Thus, we need to study the non-generic case where $L_{1}=L_{2}$.

$>\operatorname{Rmod}:=\operatorname{subs}(\mathrm{L} 2=\mathrm{L} 1, \operatorname{evalm}(\mathrm{R}))$;

$$
R \bmod :=\left[\begin{array}{cccc}
m 1 L 1 D t^{2} & m 2 L 1 D t^{2} & -1 & (M+m 1+m 2) D t^{2} \\
m 1 L 1^{2} D t^{2}-m 1 L 1 g & 0 & 0 & m 1 L 1 D t^{2} \\
0 & m 2 L 1^{2} D t^{2}-m 2 L 1 g & 0 & m 2 L 1 D t^{2}
\end{array}\right]
$$

$>$ st:=time(): Ext1mod:=Exti(Involution(Rmod, Alg), Alg, 1): time()-st;

0.959

$>$ Ext1mod;

$$
\begin{aligned}
& {\left[\begin{array}{ccc}
L 1 D t^{2}-g & 0 & 0 \\
0 & L 1 D t^{2}-g & 0 \\
0 & 0 & L 1 D t^{2}-g
\end{array}\right],\left[\begin{array}{ccrc}
1 & -1 & 0 & 0 \\
0 & m 1 g+m 2 g & -1 & D t^{2} M \\
0 & M L 1 D t^{2}-g M-m 1 g-m 2 g & 1 & 0
\end{array}\right],} \\
& {\left[\begin{array}{c}
-D t^{2} \\
-D t^{2}
\end{array}\right]} \\
& \qquad 1 D t^{4} M-D t^{2} m 1 g-g M D t^{2}-D t^{2} m 2 g \\
& L 1 D t^{2}-g
\end{aligned}
$$

The first matrix of Ext1mod is not an identity matrix, and thus, we know that $\operatorname{ext}_{D}^{1}\left(N_{\text {mod }}, D\right) \neq 0$, where $N_{\text {mod }}=D^{1 \times 3} / D^{1 \times 4} \theta\left(R_{\text {mod }}\right)$ and $R_{\text {mod }}$ corresponds to $R$, where $L_{2}=L_{1}$. Thus, the system is not controllable (see Proposition 4.2 and Theorem 8.3). The second matrix of Ext1mod gives a (non minimal) family of generators of the torsion elements of $M_{\mathrm{mod}}=D^{1 \times 4} / D^{1 \times 3} R_{\mathrm{mod}}$, i.e., of the non-controllable elements of the system. The initial rows in the first two matrices of Ext1mod shows that $d=x_{1}-x_{2}$ satisfies $\left(L_{1} \partial^{2}-g\right) d=0$ (non-controllable element). The third matrix of Ext1mod gives a parametrization of the torsion-free part $M_{\text {mod }} / t\left(M_{\bmod }\right)$ of $M_{\bmod }$, i.e., of the controllable part of the system. Finally, let us notice that it has only taken $0.959 \mathrm{~s}$ to obtain all these informations. 


\section{A.2 Linear differential algebraic equations}

Let us consider the following example of a time-varying linear OD system which corresponds to a linear system of differential algebraic equations (DAEs) studied in [16].

Therefore, we introduce the Weyl algebra $D=A_{1}$ (see Example 2.3).

$>A l g:=$ Define0reAlgebra $(\operatorname{dif} f=[D t, t], \operatorname{polynom}=\{t\}):$

The time-varying linear system is defined by means of the following matrix.

$>\mathrm{R}:=\operatorname{evalm}([[-\mathrm{t} * \mathrm{Dt}+1, \mathrm{t} \sim 2 * \mathrm{Dt},-1,0],[-\mathrm{Dt}, \mathrm{t} * \mathrm{Dt}+1,0,-1]])$;

$$
R:=\left[\begin{array}{ccrr}
-t D t+1 & t^{2} D t & -1 & 0 \\
-D t & t D t+1 & 0 & -1
\end{array}\right]
$$

Then, the system is defined by $R\left(x_{1}: x_{2}: u_{1}: u_{2}\right)^{T}=0$ and we define the left $D$-module $M=$ $D^{1 \times 4} / D^{1 \times 2} R$.

$>$ R_adj:=Involution (R, Alg);

$$
R_{-} a d j:=\left[\begin{array}{cc}
t D t+2 & D t \\
-t^{2} D t-2 t & -t D t \\
-1 & 0 \\
0 & -1
\end{array}\right]
$$

Using the procedure $\operatorname{ExT} 1\left(R_{\text {adj }}\right)$, we compute $\operatorname{ext}_{D}^{1(N, D)}$, where $N=D^{1 \times 2} / D^{1 \times 4} R_{\text {adj }}$ is the left $D$-module associated with $R_{\text {adj }}=\theta(R) \in D^{4 \times 2}$.

$>$ st:=time(): Ext1:=Exti(R_adj, Alg, 1): time()-st; Ext1[1];

$$
0.301
$$

$$
\left[\begin{array}{ll}
1 & 0 \\
0 & 1
\end{array}\right]
$$

This shows that the left $D$-module $M=D^{1 \times 4} / D^{1 \times 2} R$ is a torsion-free $D$-module (see Remark 7.9), and thus, a projective $D$-module (see 1 of Theorem 3.6).

Then, a parametrization $L_{2}$ of the system is given by the following matrix of operators.

$>\operatorname{Ext} 1[3]$

$$
\left[\begin{array}{cc}
t & 1 \\
1 & 0 \\
0 & -t D t+1 \\
0 & -D t
\end{array}\right]
$$

Thus, we obtain the parametrization $\left(x_{1}: x_{2}: x_{3}: u\right)^{T}=L_{2} z$ of $R\left(x_{1}: x_{2}: x_{3}: u\right)^{T}=0$. The system is flat, i.e., the left $D$-module $M=D^{1 \times 4} / D^{1 \times 2} R$ is free, iff the parametrization $L_{2}$ admits a left-inverse. Let us check whether or not $L_{2}$ admits a left-inverse.

$>$ LeftInverse(Ext1[3], Alg);

$$
P:=\left[\begin{array}{cccc}
0 & 1 & 0 & 0 \\
0 & 0 & 1 & -t
\end{array}\right]
$$

We see that the system is flat and a flat output is defined by $\left(z_{1}: z_{2}\right)^{T}=P\left(x_{1}: x_{2}: x_{3}: u\right)^{T}$. 


\section{A.3 Wind tunnel model}

In this example, we consider again the linear differential time-delay system (4) defined in 3.2. We first need to define the Ore algebra $D_{h}=\mathbb{R}(a, k, \zeta, \omega)[t, s]\left[\partial ; \sigma_{1}, \delta_{1}\right]\left[\delta_{h} ; \sigma_{2}, \delta_{2}\right]$ of differential time-delay operators.

$>$ Alg:=Define0reAlgebra(diff $=[D t, t]$, dual_shift $=[$ delta, $s]$, polynom $=\{t, s\}$, $>\operatorname{comm}=\{\mathrm{a}$, omega, zeta, $\mathrm{k}\})$ :

The linear differential time-delay system is defined by matrix (5).

$>\mathrm{R}:=\operatorname{evalm}([\mathrm{Dt}+\mathrm{a},-\mathrm{k} * \mathrm{a} *$ delta, 0,0$],[0, \mathrm{Dt},-1,0],[0$, omega^ 2 ,

$>$ Dt+2*zeta*omega, -omega^2]]);

$$
R:=\left[\begin{array}{cccc}
D t+a & -k a \delta & 0 & 0 \\
0 & D t & -1 & 0 \\
0 & \omega^{2} & D t+2 \zeta \omega & -\omega^{2}
\end{array}\right]
$$

$>R_{-}$adj: $=$linalg $[$transpose $](R)$;

$$
R_{-} a d j:=\left[\begin{array}{ccc}
D t+a & 0 & 0 \\
-k a \delta & D t & \omega^{2} \\
0 & -1 & D t+2 \zeta \omega \\
0 & 0 & -\omega^{2}
\end{array}\right]
$$

The Gröbner basis $G$ defined in Example 5.5 can be computed as follows.

$>$ Integrability (R_adj, Alg);

$$
\begin{aligned}
& {\left[\omega^{2} k a \delta \mu_{1}+\omega^{2} D t \mu_{2}+\omega^{2} a \mu_{2}+\omega^{2} D t^{2} \mu_{3}+\omega^{2} a D t \mu_{3}+D t^{3} \mu_{4}+2 D t^{2} \zeta \omega \mu_{4}\right.} \\
& +a D t^{2} \mu_{4}+D t \omega^{2} \mu_{4}+2 a D t \zeta \omega \mu_{4}+a \omega^{2} \mu_{4}, \lambda_{3} \omega^{2}+\mu_{4}, \\
& \omega^{2} \lambda_{2}+D t \mu_{4}+\omega^{2} \mu_{3}+2 \zeta \omega \mu_{4}, \\
& \left.\omega^{2} \lambda_{1} k a \delta+D t^{2} \mu_{4}+\omega^{2} D t \mu_{3}+2 D t \zeta \omega \mu_{4}+\omega^{2} \mu_{2}+\omega^{2} \mu_{4}, \lambda_{1} D t+\lambda_{1} a-\mu_{1}\right]
\end{aligned}
$$

The syzygy module of $D_{h}^{1 \times 4} R^{T}$ can be computed using $\operatorname{Syzygy}\left(R_{\text {adj }}\right)$ (see Example 5.5).

$>$ SyzygyModule (R_adj, Alg);

$$
\left[\omega^{2} k a \delta, D t \omega^{2}+a \omega^{2}, \omega^{2} D t^{2}+\omega^{2} a D t, D t \omega^{2}+a \omega^{2}+D t^{3}+2 D t^{2} \zeta \omega+a D t^{2}+2 a D t \zeta \omega\right]
$$

The Gröbner basis $G$ defined in Example 7.4 can be computed as follows.

$>$ Integrability(linalg[transpose](S), Alg);

$$
\begin{aligned}
& {\left[-\omega^{2} \mu_{4}+\omega^{2} \mu_{2}+D t \mu_{3}+2 \zeta \omega \mu_{3},-\mu_{3}+D t \mu_{2},-k a \delta \mu_{2}+D t \mu_{1}+a \mu_{1}, \lambda_{1} \omega^{2} k a \delta-\mu_{1},\right.} \\
& \left.\lambda_{1} D t \omega^{2}+\lambda_{1} a \omega^{2}-\mu_{2}\right]
\end{aligned}
$$

The syzygy module $D_{h}^{1 \times 4} R_{-1}$ can be computed using $\operatorname{Syzygy}\left(R_{-1}\right)$ (see Example 7.4).

$>$ L:=SyzygyModule(linalg[transpose] (S), Alg);

$$
L:=\left[\begin{array}{cccc}
D t+a & -k a \delta & 0 & 0 \\
0 & \omega^{2} & D t+2 \zeta \omega & -\omega^{2} \\
0 & D t & -1 & 0
\end{array}\right]
$$

Finally, we compute Quotient $(L, R)$ (see Example 7.7) as follows.

$>\mathrm{Q}:=$ Quotient (L, R, Alg); 


$$
Q:=\left[\begin{array}{lll}
1 & 0 & 0 \\
0 & 1 & 0 \\
0 & 0 & 1
\end{array}\right]
$$

We deduce that $\operatorname{ext}_{D_{h}}^{1}\left(N, D_{h}\right)=0$, where $N=D_{h}^{1 \times 3} / D_{h}^{1 \times 4} R^{T}$ (see Remark 7.9), and thus, system (4) is controllable and parametrizable (see Proposition 4.2 and Theorem 8.3).

We can directly compute $\operatorname{ext}_{D_{h}}^{1}\left(N, D_{h}\right)$ using $\operatorname{ExT} 1\left(R_{\text {adj }}\right)$ defined in Algorithm 7.8.

> st:=time(): Ext1:=Exti(Involution(R,Alg), Alg, 1); time()-st;

$$
\begin{aligned}
& \operatorname{Ext1}:=\left[\left[\begin{array}{lll}
1 & 0 & 0 \\
0 & 1 & 0 \\
0 & 0 & 1
\end{array}\right],\left[\begin{array}{cccc}
D t+a & -k a \delta & 0 & 0 \\
0 & \omega^{2} & D t+2 \zeta \omega & -\omega^{2} \\
0 & D t & -1 & 0
\end{array}\right],\right. \\
& \left.\left[\begin{array}{c}
-\omega^{2} k a \delta \\
-D t \omega^{2}-a \omega^{2} \\
-\omega^{2} D t^{2}-\omega^{2} a D t \\
-D t^{3}-2 D t^{2} \zeta \omega-a D t^{2}-D t \omega^{2}-2 a D t \zeta \omega-a \omega^{2}
\end{array}\right]\right]
\end{aligned}
$$

The first two matrices in Ext1 are $Q$ and $L$. Moreover, Ext1[3] gives (up to the sign) the parametrization (30) of system (4), i.e., we have $\left(x_{1}: x_{2}: x_{2}: u\right)^{T}=\operatorname{Ext} 1[3] z$, where $z$ is an arbitrary function of $t$.

We compute $\operatorname{ext}_{D_{h}}^{2}\left(N, D_{h}\right)$ in order to check whether or not system (30) is flat.

$>$ Ext2:=Exti(Involution(R, Alg), Alg, 2);

$$
\text { Ext2 }:=\left[\left[\begin{array}{c}
\delta \\
D t+a
\end{array}\right],[1], \operatorname{SURJ}(1), \operatorname{INJ}(1)\right]
$$

Since the first matrix in Ext2 is not an identity matrix, we know that $\operatorname{ext}_{D_{h}}^{2}\left(N, D_{h}\right) \neq 0$ (see Remark 7.9). Hence, $M=D_{h}^{1 \times 4} / D_{h}^{1 \times 3} R$ is a torsion-free but not a free $D_{h}$-module, and thus, (4) is not a flat system. Finally, let us notice that (14) is equivalent to the reduced system:

$$
(\partial+a) y=0, \quad \delta_{h} y=0 .
$$

Then, the formal obstructions of flatness are defined by $\pi$-POLYNOMIAL $\left(R_{\mathrm{adj}},\left\{\partial, \delta_{h}\right\}\right)$.

$>$ PiPolynomial (R, Alg);

$$
[\delta, D t+a]
$$

The $\pi$-polynomial, such that $\left(D_{h}\right)_{\pi} \otimes_{D_{h}} M$ is a free $\left(D_{h}\right)_{\pi}$-module, is defined by the generator of the principal ideal $\pi$-POLYNOMIAL $\left(R_{\text {adj }},\left\{\delta_{h}\right\}\right)$ of $\mathbb{R}(a, k, \zeta, \omega)\left[\delta_{h} ; \sigma_{2}, \delta_{2}\right]$.

$>$ PiPolynomial (R, Alg, [delta]);

Therefore, we find that system (4) is $\delta_{h}$-flat (see Example 9.6).

$>$ PiPolynomial (R, Alg, $[D t]$ );

$$
[D t+a]
$$

The fact that (4) is not a flat system is coherent with the fact that the full row-rank matrix $R$, defined by (5), does not admit a right-inverse ( $R$ admits a right-inverse iff the $D_{h}$-module $M$ is projective, and thus, free by 2 of Theorem 3.6).

> RightInverse(R, Alg); 
The fact that (4) is not a flat system is also coherent with the fact that its parametrization (30) does not admit a left-inverse (a linear system is flat iff it is parametrizable and its parametrization admits a left-inverse).

$>$ LeftInverse(Ext1[3], Alg);

But, we have shown that system (4) is $\delta_{h}$-flat, and thus, the $D_{h}\left[\delta_{h}^{-1}\right]$-module $D_{h}\left[\delta_{h}^{-1}\right] \otimes_{D_{h}} M$ is free. Let us compute a basis of the free $D_{h}\left[\delta_{h}^{-1}\right]$-module $D_{h}\left[\delta_{h}^{-1}\right] \otimes_{D_{h}} M$. In order to do that, we compute a left-inverse $S$ of the parametrization Ext1/3] in the commutative polynomial ring $D_{h}\left[\delta_{h}^{-1}\right]$.

$>\mathrm{S}:=$ LocalLeftInverse(Ext1[3],[delta],Alg);

$$
S:=\left[\begin{array}{llll}
-\frac{1}{\delta \omega^{2} k a} & 0 & 0 & 0
\end{array}\right]
$$

By construction, we have $S \operatorname{Ext} 1[3]=1$.

$>\operatorname{simplify}(\operatorname{evalm}(\mathrm{S} \& * \operatorname{Ext} 1[3]))$;

Hence, we obtain that $z=S\left(x_{1}: x_{2}: x_{3}: u\right)^{T}=-\delta_{h}^{-1} x_{1} /\left(\omega^{2} k a\right)$ is a basis of the $D_{h}\left[\delta_{h}^{-1}\right]$-module $D_{h}\left[\delta_{h}^{-1}\right] \otimes_{D_{h}} M$ because we have $\left(x_{1}: x_{2}: x_{3}: u\right)^{T}=\operatorname{Ext} 1[3] z$ and $z=-\delta_{h}^{-1} x_{1} /\left(\omega^{2} k a\right) \in M$.

Let us finally point out that we can also substitute $z=-\delta_{h}^{-1} x_{1} /\left(\omega^{2} k a\right)$ into the parametrization $\left(x_{1}: x_{2}: x_{3}: u\right)^{T}=\operatorname{Ext} 1[3] z$ of system (4) in order to express the system variables in terms of $x_{1}$.

$>\mathrm{T}:=\operatorname{simplify}(\operatorname{evalm}(\operatorname{Ext} 1[3] \& * \mathrm{~S}))$;

$$
T:=\left[\begin{array}{cccc}
1 & 0 & 0 & 0 \\
\frac{D t+a}{k a \delta} & 0 & 0 & 0 \\
\frac{D t(D t+a)}{k a \delta} & 0 & 0 & 0 \\
\frac{D t \omega^{2}+a \omega^{2}+D t^{3}+2 D t^{2} \zeta \omega+a D t^{2}+2 a D t \zeta \omega}{\omega^{2} k a \delta} & 0 & 0 & 0
\end{array}\right]
$$

We obtain $\left(x_{1}: x_{2}: x_{3}: u\right)^{T}=T x_{1}$, and thus, $x_{1}$ is also a basis of the module $D_{h}\left[\delta_{h}^{-1}\right] \otimes_{D_{h}} M$ over $D_{h}\left[\delta_{h}^{-1}\right]$, and thus, a flat output of system (4) over $D_{h}\left[\delta_{h}^{-1}\right]$. To finish, let us notice that, by imposing $x_{1}$ to be a desired trajectory $x_{1 d}(t)$, we obtain the corresponding open-loop input [20]:

$$
u_{d}(t)=\frac{1}{\omega^{2} a k}\left(\left(\frac{d}{d t}^{2}+2 \zeta \omega \frac{d}{d t}+\omega^{2}\right)\left(\frac{d}{d t}+a\right)\right) x_{1 d}(t+h) .
$$

\section{A.4 A two reflector antenna}

Let us consider the example of a two reflector antenna [20]. Any linear differential constant time-delay systems can be studied similarly. We refer to [20,21] for more examples.

$>A l g:=$ DefineOreAlgebra $\left(\operatorname{dif} f=[D t, t], \operatorname{dual} \_\right.$shift $=[\operatorname{delta}, \mathrm{s}]$, polynom=\{t,s\},

$>\mathrm{comm}=\{\mathrm{K} 1, \mathrm{~K} 2, \mathrm{Te}, \mathrm{Kp}, \mathrm{Kc}\})$ :

The system of the two reflector antenna is defined by the following matrix with entries in the commutative polynomial ring $D_{h}=\mathbb{R}(K 1, K 2, T e, K p, K c)\left[\partial ; \sigma_{1}, \delta_{1}\right]\left[\delta_{h} ; \sigma_{2}, \delta_{2}\right]$ of differential time-delay operators. 


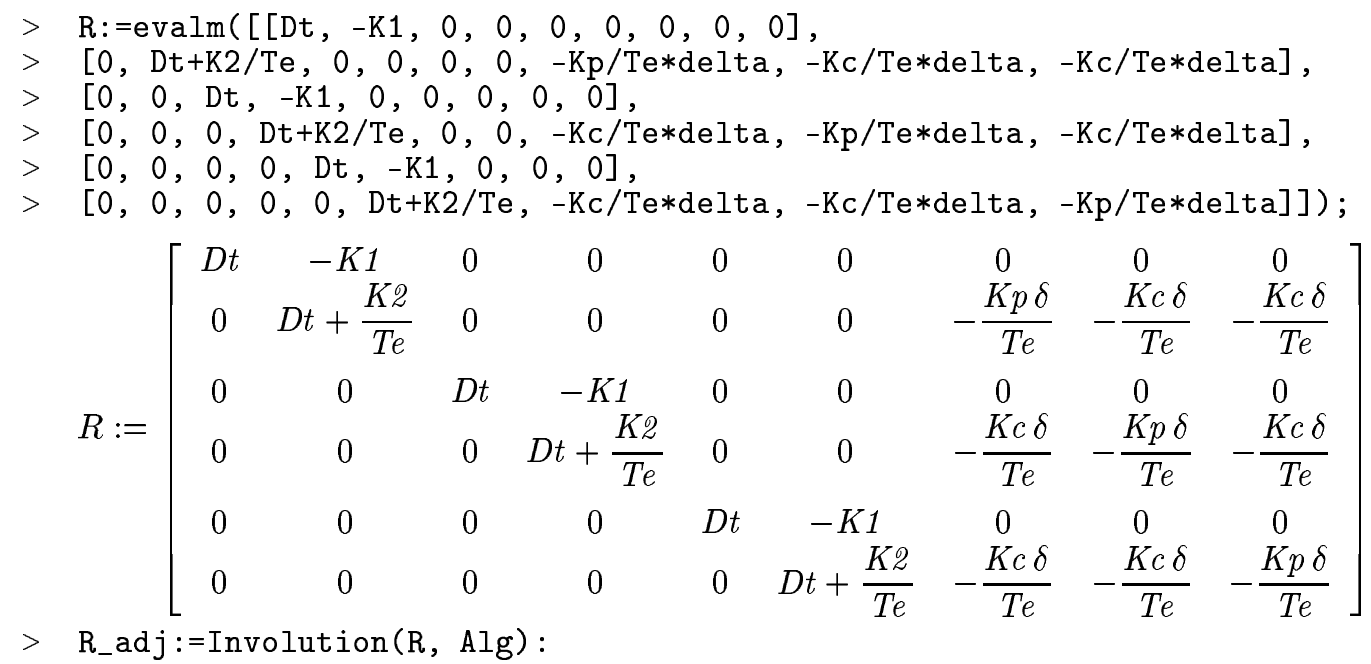

Let us check whether or not the system, defined by the matrix $R$, is controllable and, if it is the case, let us find one of its parametrizations. In order to do that, we need to compute $\operatorname{ext}_{D_{h}}^{1}\left(N, D_{h}\right)$, where $N=D_{h}^{1 \times 6} / D_{h}^{1 \times 9} R$. The displayed result corresponds to $L_{0}$ in the output of ExT1.

$>$ st:=time(): Ext1:=Exti $\left(R_{-}\right.$adj, Alg, 1): time()-st; Ext1[1];

2.410

$$
\left[\begin{array}{llllll}
1 & 0 & 0 & 0 & 0 & 0 \\
0 & 1 & 0 & 0 & 0 & 0 \\
0 & 0 & 1 & 0 & 0 & 0 \\
0 & 0 & 0 & 1 & 0 & 0 \\
0 & 0 & 0 & 0 & 1 & 0 \\
0 & 0 & 0 & 0 & 0 & 1
\end{array}\right]
$$

We obtain that $t(M)=\operatorname{ext}_{D_{h}}^{1}\left(N, D_{h}\right)=0$ (see Remark 7.9), where $M=D_{h}^{1 \times 9} / D_{h}^{1 \times 6} R$, and thus, the system of two reflector antenna is controllable (see Proposition 4.2 and Theorem 8.3).

Then, the matrix $L_{2}$, defined by

$>\operatorname{Ext1}[3]$

$\left[\begin{array}{ccc}K c \delta K 1 & K c \delta K 1 & K p K 1 \delta \\ K c D t \delta & K c D t \delta & K p D t \delta \\ K c \delta K 1 & K p K 1 \delta & K c \delta K 1 \\ K c D t \delta & K p D t \delta & K c D t \delta \\ K p K 1 \delta & K c \delta K 1 & K c \delta K 1 \\ K p D t \delta & K c D t \delta & K c D t \delta \\ 0 & 0 & D t^{2} T e+K 2 D t \\ 0 & D t^{2} T e+K 2 D t & 0 \\ D t^{2} T e+K 2 D t & 0 & 0\end{array}\right]$

is a parametrization of the two reflector antenna. The two reflector antenna is not a flat system because we have $\operatorname{ext}_{D_{h}}^{2}\left(N, D_{h}\right) \neq 0$ as it is shown below.

$>$ st:=time(): Ext2:=Exti(R_adj, Alg, 2): time()-st; Ext2[1];

1.931

$$
\left[\begin{array}{ccc}
\delta & 0 & 0 \\
D t^{2} T e+K 2 D t & 0 & 0 \\
0 & \delta & 0 \\
0 & D t^{2} T e+K 2 D t & 0 \\
0 & 0 & \delta \\
0 & 0 & D t^{2} T e+K 2 D t
\end{array}\right]
$$


Since the torsion-free degree $i(M)$ of $M$ is equal to 1 (see Proposition 9.4), we can find a polynomial $\pi$ containing only one of the indeterminates $D t$ and $\delta$ such that $\left(D_{h}\right)_{\pi} \otimes_{D_{h}} M$ is a free $\left(D_{h}\right)_{\pi}$-module. We study polynomials in $\delta_{h}$.

$>$ PiPolynomial (R, Alg, [delta]);

So, we conclude that the two reflector antenna is $\delta_{h}$-free [20]. Let us compute a basis of the $D_{h}\left[\delta_{h}^{-1}\right]$ module $D_{h}\left[\delta_{h}^{-1}\right] \otimes_{D_{h}} M$. In order to do that, let us compute a left-inverse $S$ of Ext1[3] in $D_{h}\left[\delta_{h}^{-1}\right]$.

$>\mathrm{S}:=$ LocalLeftInverse(Ext1[3], [delta], Alg);

$$
\begin{aligned}
& S:=\left[\begin{array}{ccccccccc}
-\frac{K c}{\delta K 1 \% 1} & 0 & -\frac{K c}{\delta K 1 \% 1} & 0 & \frac{K p+K c}{\delta K 1 \% 1} & 0 & 0 & 0 & 0 \\
-\frac{K c}{\delta K 1 \% 1} & 0 & \frac{K p+K c}{\delta K 1 \% 1} & 0 & -\frac{K c}{\delta K 1 \% 1} & 0 & 0 & 0 & 0 \\
\frac{K p+K c}{\delta K 1 \% 1} & 0 & -\frac{K c}{\delta K 1 \% 1} & 0 & -\frac{K c}{\delta K 1 \% 1} & 0 & 0 & 0 & 0
\end{array}\right] \\
& \% 1:=K p^{2}-2 K c^{2}+K p K c
\end{aligned}
$$

We easily check that $S \in D_{h}\left[\delta_{h}^{-1}\right]^{3 \times 9}$ satisfies $S \operatorname{Ext} 1[3]=I_{3}$.

$>\operatorname{simplify}($ evalm $(\mathrm{S} \& * \operatorname{Ext} 1[3]))$;

$$
\left[\begin{array}{lll}
1 & 0 & 0 \\
0 & 1 & 0 \\
0 & 0 & 1
\end{array}\right]
$$

Hence, in the $D_{h}\left[\delta_{h}^{-1}\right]$-module $D_{h}\left[\delta_{h}^{-1}\right] \otimes_{D_{h}} M$, we have

$$
\left(z_{1}: z_{2}: z_{3}\right)^{T}=S\left(x_{1}: \ldots: x_{6}: u_{1}: u_{2}: u_{3}\right)^{T},
$$

and thus, $\left\{z_{1}, z_{2}, z_{3}\right\}$ is a basis of the $D_{h}\left[\delta_{h}^{-1}\right]$-module $D_{h}\left[\delta_{h}^{-1}\right] \otimes_{D_{h}} M$ which satisfies:

$$
\left(x_{1}: \ldots: x_{6}: u_{1}: u_{2}: u_{3}\right)^{T}=\operatorname{Ext} 1[3]\left(z_{1}: z_{2}: z_{3}\right)^{T} .
$$

Finally, if we substitute (42) into the parametrization Ext1[3] of the system, we obtain

$$
\left(x_{1}: \ldots: x_{6}: u_{1}: u_{2}: u_{3}\right)^{T}=T\left(x_{1}: \ldots: x_{6}: u_{1}: u_{2}: u_{3}\right)^{T},
$$

where the matrix $T \in D_{h}\left[\delta_{h}^{-1}\right]^{9 \times 9}$ is defined by:

$>\mathrm{T}:=\operatorname{simplify}($ evalm $(\operatorname{Ext} 1[3] \& * \mathrm{~S}))$; 


$$
\begin{aligned}
& T:=\left[\begin{array}{c}
1,0,0,0,0,0,0,0,0 \\
\frac{D t}{K 1}, 0,0,0,0,0,0,0,0 \\
0,0,1,0,0,0,0,0,0 \\
0,0, \frac{D t}{K 1}, 0,0,0,0,0,0 \\
0,0,0,0,1,0,0,0,0 \\
0,0,0,0, \frac{D t}{K 1}, 0,0,0,0 \\
\frac{D t(T e D t+K 2)(K p+K c)}{\delta K 1 \% 1}, 0, \% 2,0, \% 2,0,0,0,0 \\
\% 2,0, \frac{D t(T e D t+K 2)(K p+K c)}{\delta K 1 \% 1}, 0, \% 2,0,0,0,0 \\
\% 2,0, \% 2,0, \frac{D t(T e D t+K 2)(K p+K c)}{\delta K 1 \% 1}, 0,0,0,0
\end{array}\right] \\
& \% 1:=K p^{2}-2 K c^{2}+K p K c \\
& =-\frac{D t(T e D t+K 2) K c}{\delta K 1 \% 1}
\end{aligned}
$$

Then, we notice $\left(x_{2}: x_{4}: x_{6}: u_{1}: u_{2}: u_{3}\right)$ are only expressed in terms of $x_{1}, x_{3}$ and $x_{5}$. Therefore, $\left\{x_{1}, x_{3}, x_{5}\right\}$ is also a basis of the $D_{h}\left[\delta_{h}^{-1}\right]$-module $D_{h}\left[\delta_{h}^{-1}\right] \otimes_{D_{h}} M$ of rank 3 (compare with [20]).

\section{A.5 An electric transmission line}

To finish with linear differential time-delay systems, we shall study the example of an electric transmission line [34]. We shall exhibit an explict parametrization of this system. It seems that no parametrization for such a system was previously known [20]. This can be easily explained by the fact that it was very difficult to guess such a parametrization (indeed, the characterization in terms of the extension functor of the existence of a parametrization has only been recently developed in [27]). Moreover, it becomes very difficult to avoid the use of a symbolic computation program in order to handle all the computations.

We first introduce $D_{h}=\mathbb{R}\left(a_{0}, a_{1}, a_{2}, a_{3}, a_{4}, a_{5}, b_{0}\right)[t, s]\left[\partial ; \sigma_{1}, \delta_{1}\right]\left[\delta_{h} ; \sigma_{2}, \delta_{2}\right]$, the Ore algebra of differential time-delay operators.

$>A l g:=$ Define0reAlgebra (diff $=[D t, t]$, dual_shift $=[$ delta, $s]$, polynom $=\{t, s\}$,

$>\quad c o m m=\{a[0], a[1], a[2], a[3], a[4], a[5], b[0]\}):$

The electric transmission line is defined by means of the following matrix:

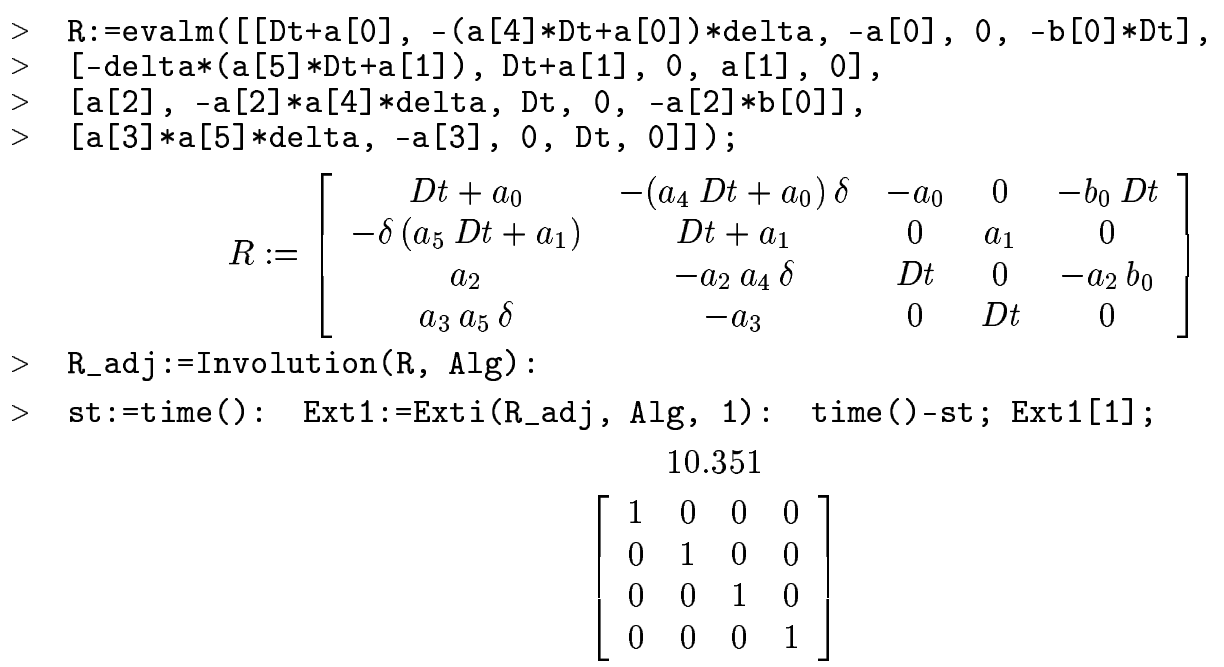


Therefore, by Proposition 4.2 and Theorem 8.3, we find that the electric transmission line is controllable and parametrizable and a parametrization is given by the following matrix.

$>\operatorname{Ext1}[3]$

$$
\begin{aligned}
& {\left[-b_{0} D t^{4}-D t^{2} b_{0} a_{1} a_{3}-a_{0} a_{2} b_{0} D t^{2}-a_{1} a_{0} a_{2} b_{0} a_{3}-a_{0} a_{1} a_{2} b_{0} D t-a_{1} b_{0} D t^{3}\right]} \\
& {\left[-a_{5} b_{0} \delta D t^{4}-a_{5} \delta D t^{2} b_{0} a_{1} a_{3}-a_{1} b_{0} \delta D t^{3}-a_{0} a_{2} a_{5} b_{0} \delta D t^{2}\right.} \\
& \left.-a_{0} a_{1} a_{2} a_{5} a_{3} \delta b_{0}-a_{0} a_{1} a_{2} b_{0} \delta D t\right] \\
& {\left[a_{0} a_{2} a_{1} b_{0} a_{3} a_{5} \delta^{2}-a_{0} a_{2} b_{0} D t^{2}-a_{1} a_{0} a_{2} b_{0} a_{3}+a_{0} a_{1} a_{2} b_{0} \delta^{2} D t\right.} \\
& \left.-a_{0} a_{1} a_{2} b_{0} D t+a_{0} a_{2} a_{5} \delta^{2} b_{0} D t^{2}\right] \\
& {\left[a_{0} a_{1} a_{2} a_{5} a_{3} \delta b_{0}-\delta D t^{2} b_{0} a_{1} a_{3}+a_{5} \delta D t^{2} b_{0} a_{1} a_{3}-a_{0} a_{1} a_{2} a_{3} \delta b_{0}\right]} \\
& {\left[-D t^{4}-a_{1} D t^{3}-a_{0} a_{1} D t^{2}-a_{0} D t^{3}+a_{5} D t^{4} \delta^{2} a_{4}+a_{1} a_{5} D t^{2} a_{3} a_{4} \delta^{2}+a_{1} D t^{3} a_{4} \delta^{2}\right.} \\
& +a_{0} a_{2} a_{5} D t^{2} a_{4} \delta^{2}+a_{0} D t^{3} \delta^{2} a_{5}-a_{1} a_{3} a_{0} a_{2}-a_{0} a_{1} a_{2} D t-a_{0} a_{1} a_{3} D t \\
& +a_{0} a_{1} D t^{2} \delta^{2}+a_{0} a_{1} a_{2} a_{5} a_{3} a_{4} \delta^{2}+a_{0} a_{1} a_{2} D t a_{4} \delta^{2}+a_{0} a_{1} a_{3} \delta^{2} D t a_{5} \\
& \left.-a_{1} D t^{2} a_{3}-a_{0} a_{2} D t^{2}\right] \\
& >\text { st:=time(): Ext2:=Exti(R_adj, Alg, 2): time()-st; Ext2[1]; } \\
& 6.990 \\
& {\left[-\delta a_{1}^{2} a_{5} a_{3}^{2}+2 a_{0} a_{1} a_{2} a_{5} a_{3} \delta-D t \delta a_{2} a_{1} a_{0}+\delta D t a_{1}^{2} a_{3}+\delta a_{5} a_{2} a_{0} D t a_{1}\right.} \\
& -\delta a_{1}^{2} a_{5} a_{3} D t+\delta^{3} a_{1}^{2} a_{2} a_{0}-2 a_{5}^{2} a_{2} a_{0} a_{1} a_{3} \delta^{3}+a_{5}{ }^{2} a_{2}{ }^{2} a_{0}{ }^{2} \delta^{3}+a_{1}^{2} a_{5}^{2} a_{3}^{2} \delta^{3} \\
& \left.-a_{5} a_{2}^{2} a_{0}^{2} \delta-a_{1}^{2} \delta a_{2} a_{0}\right] \\
& {\left[D t \delta^{2} a_{1}-D t^{2}+a_{1} a_{3} a_{5} \delta^{2}-D t a_{1}-a_{1} a_{3}-a_{5} \delta^{2} a_{2} a_{0}\right]} \\
& {\left[\delta a_{2} a_{0}+\delta D t^{2}\right]} \\
& {\left[a_{1} D t^{3}+D t^{2} a_{1}^{2}+a_{0} a_{2} a_{5} D t^{2}-a_{5} D t^{2} a_{1} a_{3}+\delta^{2} a_{1}{ }^{2} a_{2} a_{0}-2 a_{5}^{2} a_{2} a_{0} a_{1} a_{3} \delta^{2}\right.} \\
& +a_{5}^{2} a_{2}^{2} a_{0}^{2} \delta^{2}+a_{1}^{2} a_{5}^{2} a_{3}^{2} \delta^{2}+D t a_{1}^{2} a_{3}+a_{5} a_{2} a_{0} D t a_{1}-a_{1}^{2} a_{5} a_{3} D t \\
& \left.+a_{1} a_{5} a_{3} a_{2} a_{0}-a_{1}{ }^{2} a_{5} a_{3}{ }^{2}\right]
\end{aligned}
$$

Since we have $\operatorname{ext}_{D_{h}}^{2}\left(N, D_{h}\right) \neq 0$, where $N=D_{h}^{1 \times 4} / D_{h}^{1 \times 5} R^{T}$, the transmission line is not a flat system. Thus, we have $i(M)=1$, and we can find a polynomial $\pi$ that contains only $D t$ or $\delta$ such that $\left(D_{h}\right)_{\pi} \otimes_{D_{h}} M$ is a free $\left(D_{h}\right)_{\pi}$-module. The third argument for $\pi$-POLYNOMIAL selects the variable for the $\pi$-polynomial:

$$
\begin{aligned}
>\text { pi: } & =\text { PiPolynomial (R, Alg, [delta]) : factor (pi) } \\
& {\left[\delta \left(a_{5}^{2} a_{2}^{2} a_{0}^{2} \delta^{4}-2 \delta^{2} a_{5} a_{2}^{2} a_{0}^{2}+a_{2}^{2} a_{0}^{2}-2 a_{5}^{2} a_{2} a_{0} a_{1} a_{3} \delta^{4}+4 \delta^{2} a_{1} a_{5} a_{3} a_{2} a_{0}\right.\right.} \\
& +a_{1}^{2} a_{2} a_{0}-2 \delta^{2} a_{1}^{2} a_{2} a_{0}-2 a_{1} a_{3} a_{0} a_{2}+\delta^{4} a_{1}^{2} a_{2} a_{0}+a_{1}{ }^{2} a_{5}{ }^{2} a_{3}^{2} \delta^{4} \\
& \left.\left.-2 \delta^{2} a_{1}^{2} a_{5} a_{3}^{2}+a_{1}^{2} a_{3}^{2}\right)\right]
\end{aligned}
$$

We conclude that the system is $\pi$-free, where $\pi$ is the previous polynomial in $\delta_{h}$, and thus, the $D_{h}\left[\pi^{-1}\right]$ module $D_{h}\left[\pi^{-1}\right] \otimes_{D_{h}} M$ is free. Let us compute a basis of the $D_{h}\left[\pi^{-1}\right]$-module $D_{h}\left[\pi^{-1}\right] \otimes_{D_{h}} M$.

$>\mathrm{S}:=$ LocalLeftInverse(Ext1[3],pi,Alg):

$>\mathrm{T}:=\operatorname{map}(\operatorname{collect}, \mathrm{S}, \operatorname{delta})$; 


$$
\begin{aligned}
& T:= \\
& {\left[-\frac{\delta\left(\delta^{4} a_{0} a_{5}^{2}+\left(-a_{5} a_{0}-a_{0} a_{5}^{2}+a_{1}-a_{1} a_{5}\right) \delta^{2}-a_{1}+a_{1} a_{5}+a_{5} a_{0}\right)}{\% 2 a_{0} b_{0}\left(-1+a_{5}\right)},\right.} \\
& \frac{\left(a_{5} a_{0}-a_{1} a_{5} a_{4}+a_{1} a_{4}\right) \delta^{4}+\left(-a_{5} a_{0}+a_{1} a_{5} a_{4}-a_{1} a_{4}-a_{0}\right) \delta^{2}+a_{0}}{\% 2 a_{0} b_{0}\left(-1+a_{5}\right)}, \\
& -\frac{\delta\left(\left(-a_{1} a_{5} a_{3}+a_{5} a_{2} a_{0}\right) \delta^{2}-a_{0} a_{2}+a_{1} a_{3}\right)}{\% 2 a_{0} a_{2} b_{0}}, \\
& \frac{\left(a_{0} a_{5}^{2} a_{2}-a_{5}^{2} a_{1} a_{3}+a_{1}^{2}\right) \delta^{4}+\left(2 a_{1} a_{5} a_{3}-2 a_{5} a_{2} a_{0}-2 a_{1}^{2}\right) \delta^{2}+a_{1}^{2}+a_{0} a_{2}-a_{1} a_{3}}{\% 2 a_{1} a_{3} b_{0}\left(-1+a_{5}\right)} \\
& \left.-\frac{\delta a_{1}\left(\delta^{2}-1\right)}{\% 2 a_{0}}\right] \\
& \% 1:=a_{1}^{2} a_{2} a_{0} \\
& \% 2:=\left(a_{0}^{2} a_{2}^{2} a_{5}^{2}+\% 1-2 a_{5}^{2} a_{1} a_{3} a_{2} a_{0}+a_{5}^{2} a_{1}^{2} a_{3}^{2}\right) \delta^{5} \\
& +\left(-2 a_{5} a_{1}^{2} a_{3}^{2}+4 a_{0} a_{1} a_{2} a_{5} a_{3}-2 a_{1}^{2} a_{2} a_{0}-2 a_{0}^{2} a_{5} a_{2}^{2}\right) \delta^{3} \\
& +\left(\% 1-2 a_{1} a_{3} a_{2} a_{0}+a_{2}^{2} a_{0}^{2}+a_{1}^{2} a_{3}^{2}\right) \delta
\end{aligned}
$$

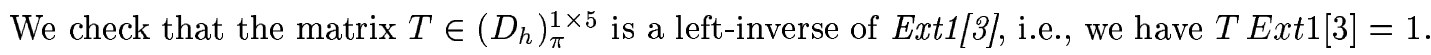

$>$ simplify (evalm $(T \& * \operatorname{Ext} 1[3]))$;

$$
[1]
$$

Hence, $z=T\left(x_{1}: \ldots: x_{4}: u\right)^{T}$ is a basis of the $D_{h}\left[\pi^{-1}\right]$-module $D_{h}\left[\pi^{-1}\right] \otimes_{D_{h}} M$ which satisfies $\left(x_{1}: \ldots: x_{4}: u\right)^{T}=\operatorname{Ext} 1[3] z$.

\section{A.6 Einstein equations}

Let us show that the results exposed in this paper can also be interesting for the study of underdetermined systems of PDEs coming from mathematical physics. We shall study the parametrizability of the Einstein equations using the linearized Ricci equations in the vacuum [24] (see also [37]).

Let us introduce the Weyl algebra $D=A_{4}\left(x_{1}, x_{2}\right.$, and $x_{3}$ stand for the three space components and $x_{4}=c t$ for the time $t$ component up to the speed of light factor).

$>A l g:=$ Def ine0reAlgebra (diff $=[D 1, x 1], \operatorname{diff}=[D 2, x 2], \operatorname{diff}=[D 3, x 3]$,

$>\operatorname{diff}=[D 4, x 4]$, polynom $=\{x 1, x 2, x 3, x 4\})$ :

The linearized Ricci equations in the vacuum are defined by the following $10 \times 10$ matrix of partial differential operators.

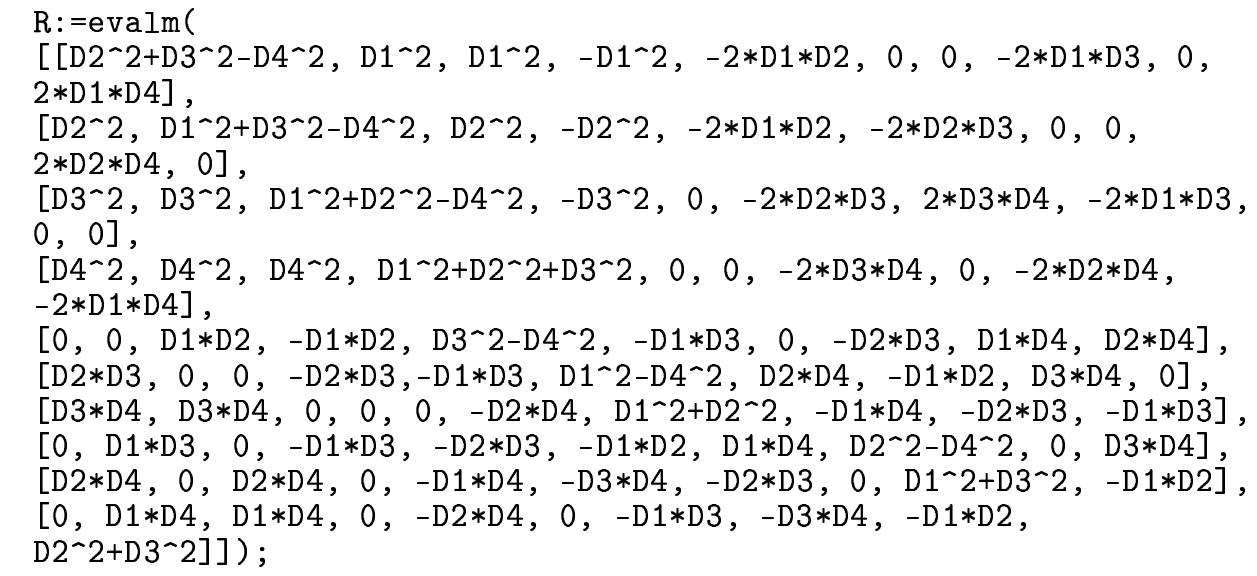




$$
\begin{aligned}
& {\left[\mathrm{D} 2^{2}+\mathrm{D} 3^{2}-\mathrm{D} 4^{2}, \mathrm{D} 1^{2}, \mathrm{D} 1^{2},-\mathrm{D} 1^{2},-2 \mathrm{D} 1 \mathrm{D} 2,0,0,-2 \mathrm{D} 1 \mathrm{D} 3,0,2 \mathrm{D} 1 \mathrm{D} 4\right]} \\
& \mathrm{D} 2^{2}, \mathrm{D} 1^{2}+\mathrm{D} 3^{2}-\mathrm{D} 4^{2}, \mathrm{D} 2^{2},-\mathrm{D} 2^{2},-2 \mathrm{D} 1 \mathrm{D} 2,-2 \mathrm{D} 2 \mathrm{D} 3,0,0,2 \mathrm{D} 2 \mathrm{D} 4,0 \\
& \mathrm{D} 3^{2}, \mathrm{D} 3^{2}, \mathrm{D} 1^{2}+\mathrm{D} 2^{2}-\mathrm{D} 4^{2},-\mathrm{D} 3^{2}, 0,-2 \mathrm{D} 2 \mathrm{D} 3,2 \mathrm{D} 3 \mathrm{D} 4,-2 \mathrm{D} 1 \mathrm{D} 3,0,0 \\
& \mathrm{D} 4^{2}, \mathrm{D} 4^{2}, \mathrm{D} 4^{2}, \mathrm{D} 1^{2}+\mathrm{D} 2^{2}+\mathrm{D} 3^{2}, 0,0,-2 \mathrm{D} 3 \mathrm{D} 4,0,-2 \mathrm{D} 2 \mathrm{D} 4,-2 \mathrm{D} 1 \mathrm{D} 4 \\
& R:=\quad 0,0, \mathrm{D} 1 \mathrm{D} 2,-\mathrm{D} 1 \mathrm{D} 2, \mathrm{D} 3^{2}-\mathrm{D} 4^{2},-\mathrm{D} 1 \mathrm{D} 3,0,-\mathrm{D} 2 \mathrm{D} 3, \mathrm{D} 1 \mathrm{D} 4, \mathrm{D} 2 \mathrm{D} 4 \\
& \text { D2 D3, 0, 0, -D2 D3, -D1 D3, D1 }{ }^{2}-\mathrm{D} 4^{2}, \mathrm{D} 2 \mathrm{D} 4,-\mathrm{D} 1 \mathrm{D} 2, \mathrm{D} 3 \mathrm{D} 4,0 \\
& \text { D3 D4, D3 D4, 0, 0, 0, -D2 D4, D1 }{ }^{2}+\mathrm{D} 2^{2},-\mathrm{D} 1 \mathrm{D} 4,-\mathrm{D} 2 \mathrm{D} 3,-\mathrm{D} 1 \mathrm{D} 3 \\
& \text { 0, D1 D3 , 0,-D1 D3, -D2 D3, -D1 D2, D1 D4, D2 }{ }^{2}-\mathrm{D} 4^{2}, 0, \mathrm{D} 3 \mathrm{D} 4 \\
& \text { D2 D4, 0, D2 D4 , 0, -D1 D4, -D3 D4, -D2 D3 , 0, D1 }{ }^{2}+\mathrm{D} 3^{2},-\mathrm{D} 1 \mathrm{D} 2 \\
& \text { 0, D1 D4, D1 D4, 0,-D2 D4, 0,-D1 D3, -D3 D4, -D1 D2, D2 }{ }^{2}+\mathrm{D} 3^{2} \\
& >R_{-} \text {adj: }=\text { Involution }(\mathrm{R}, \mathrm{Alg}) \text {; } \\
& R_{-} a d j:=\left[\begin{array}{c}
\mathrm{D} 2^{2}+\mathrm{D} 3^{2}-\mathrm{D} 4^{2}, \mathrm{D} 2^{2}, \mathrm{D} 3^{2}, \mathrm{D} 4^{2}, 0, \mathrm{D} 2 \mathrm{D} 3, \mathrm{D} 3 \mathrm{D} 4,0, \mathrm{D} 2 \mathrm{D} 4,0 \\
\mathrm{D} 1^{2}, \mathrm{D} 1^{2}+\mathrm{D}^{2}-\mathrm{D} 4^{2}, \mathrm{D} 3^{2}, \mathrm{D} 4^{2}, 0,0, \mathrm{D} 3 \mathrm{D} 4, \mathrm{D} 1 \mathrm{D} 3,0, \mathrm{D} 1 \mathrm{D} 4 \\
\mathrm{D} 1^{2}, \mathrm{D} 2^{2}, \mathrm{D} 1^{2}+\mathrm{D} 2^{2}-\mathrm{D} 4^{2}, \mathrm{D} 4^{2}, \mathrm{D} 1 \mathrm{D} 2,0,0,0, \mathrm{D} 2 \mathrm{D} 4, \mathrm{D} 1 \mathrm{D} 4 \\
-\mathrm{D} 1^{2},-\mathrm{D} 2^{2},-\mathrm{D} 3^{2}, \mathrm{D} 1^{2}+\mathrm{D} 2^{2}+\mathrm{D} 3^{2},-\mathrm{D} 1 \mathrm{D} 2,-\mathrm{D} 2 \mathrm{D} 3,0,-\mathrm{D} 1 \mathrm{D} 3,0,0 \\
-2 \mathrm{D} 1 \mathrm{D} 2,-2 \mathrm{D} 1 \mathrm{D} 2,0,0, \mathrm{D} 3^{2}-\mathrm{D} 4^{2},-\mathrm{D} 1 \mathrm{D} 3,0,-\mathrm{D} 2 \mathrm{D} 3,-\mathrm{D} 1 \mathrm{D} 4,-\mathrm{D} 2 \mathrm{D} 4 \\
0,-2 \mathrm{D} 2 \mathrm{D} 3,-2 \mathrm{D} 2 \mathrm{D} 3,0,-\mathrm{D} 1 \mathrm{D} 3, \mathrm{D} 1^{2}-\mathrm{D} 4^{2},-\mathrm{D} 2 \mathrm{D} 4,-\mathrm{D} 1 \mathrm{D} 2,-\mathrm{D} 3 \mathrm{D} 4,0 \\
0,0,2 \mathrm{D} 3 \mathrm{D} 4,-2 \mathrm{D} 3 \mathrm{D} 4,0, \mathrm{D} 2 \mathrm{D} 4, \mathrm{D} 1^{2}+\mathrm{D} 2^{2}, \mathrm{D} 1 \mathrm{D} 4,-\mathrm{D} 2 \mathrm{D} 3,-\mathrm{D} 1 \mathrm{D} 3 \\
-2 \mathrm{D} 1 \mathrm{D} 3,0,-2 \mathrm{D} 1 \mathrm{D} 3,0,-\mathrm{D} 2 \mathrm{D} 3,-\mathrm{D} 1 \mathrm{D} 2,-\mathrm{D} 1 \mathrm{D} 4, \mathrm{D} 2^{2}-\mathrm{D} 4^{2}, 0,-\mathrm{D} 3 \mathrm{D} 4 \\
0,2 \mathrm{D} 2 \mathrm{D} 4,0,-2 \mathrm{D} 2 \mathrm{D} 4, \mathrm{D} 1 \mathrm{D} 4, \mathrm{D} 3 \mathrm{D} 4,-\mathrm{D} 2 \mathrm{D} 3,0, \mathrm{D} 1^{2}+\mathrm{D} 3^{2},-\mathrm{D} 1 \mathrm{D} 2 \\
2 \mathrm{D} 1 \mathrm{D} 4,0,0,-2 \mathrm{D} 1 \mathrm{D} 4, \mathrm{D} 2 \mathrm{D} 4,0,-\mathrm{D} 1 \mathrm{D} 3, \mathrm{D} 3 \mathrm{D} 4,-\mathrm{D} 1 \mathrm{D} 2, \mathrm{D} 2^{2}+\mathrm{D} 3^{2}
\end{array}\right]
\end{aligned}
$$

Let us study whether or not the linearized Ricci equations are parametrizable. Let us notice that this problem is related to a question asked by J. Wheeler on the existence of potentials for the Einstein equations [24].

$>$ st:=time(): Ext1:=Exti(R_adj, Alg, 1): time()-st; Ext1[1];

86.810

$$
\begin{aligned}
& {\left[\begin{array}{c}
\% 1,0,0,0,0,0,0,0,0,0,0,0,0,0,0,0,0,0,0,0 \\
0, \% 1,0,0,0,0,0,0,0,0,0,0,0,0,0,0,0,0,0,0 \\
0,0, \% 1,0,0,0,0,0,0,0,0,0,0,0,0,0,0,0,0,0 \\
0,0,0, \% 1,0,0,0,0,0,0,0,0,0,0,0,0,0,0,0,0 \\
0,0,0,0, \% 1,0,0,0,0,0,0,0,0,0,0,0,0,0,0,0 \\
0,0,0,0,0, \% 1,0,0,0,0,0,0,0,0,0,0,0,0,0,0 \\
0,0,0,0,0,0, \% 1,0,0,0,0,0,0,0,0,0,0,0,0,0 \\
0,0,0,0,0,0,0, \% 1,0,0,0,0,0,0,0,0,0,0,0,0 \\
0,0,0,0,0,0,0,0, \% 1,0,0,0,0,0,0,0,0,0,0,0 \\
0,0,0,0,0,0,0,0,0, \% 1,0,0,0,0,0,0,0,0,0,0 \\
0,0,0,0,0,0,0,0,0,0, \% 1,0,0,0,0,0,0,0,0,0 \\
0,0,0,0,0,0,0,0,0,0,0, \% 1,0,0,0,0,0,0,0,0 \\
0,0,0,0,0,0,0,0,0,0,0,0, \% 1,0,0,0,0,0,0,0 \\
0,0,0,0,0,0,0,0,0,0,0,0,0, \% 1,0,0,0,0,0,0 \\
0,0,0,0,0,0,0,0,0,0,0,0,0,0, \% 1,0,0,0,0,0 \\
0,0,0,0,0,0,0,0,0,0,0,0,0,0,0, \% 1,0,0,0,0 \\
0,0,0,0,0,0,0,0,0,0,0,0,0,0,0,0, \% 1,0,0,0 \\
0,0,0,0,0,0,0,0,0,0,0,0,0,0,0,0,0, \% 1,0,0 \\
0,0,0,0,0,0,0,0,0,0,0,0,0,0,0,0,0,0, \% 1,0 \\
0,0,0,0,0,0,0,0,0,0,0,0,0,0,0,0,0,0,0, \% 1
\end{array}\right]} \\
& \% 1:=\mathrm{D} 3^{2}+\mathrm{D} 2^{2}-\mathrm{D} 4^{2}+\mathrm{D} 1^{2}
\end{aligned}
$$

Therefore, we see that the linearized Ricci equations are not parametrizable because the system, defined by $R \in D^{10 \times 10}$, admits a family of 20 torsion elements which generate the torsion submodule of the $D$-module $M=D^{1 \times 10} / D^{1 \times 10} R$. Let us notice that every torsion element of the system satisfies the Dalembertian equation, namely $\left(\triangle-c^{2} \frac{\partial^{2}}{\partial t^{2}}\right) y=0$ (travelling wave in space-time).

The list of 20 torsion elements of the system is given by the following $20 \times 10$ matrix. 


$$
\left[\begin{array}{c}
\mathrm{D} 2 \mathrm{D} 4,0,0,0,-\mathrm{D} 1 \mathrm{D} 4,0,0,0, \mathrm{D} 1^{2},-\mathrm{D} 1 \mathrm{D} 2 \\
\mathrm{D} 3 \mathrm{D} 4,0,0,0,0,0, \mathrm{D} 1^{2},-\mathrm{D} 1 \mathrm{D} 4,0,-\mathrm{D} 1 \mathrm{D} 3 \\
\mathrm{D} 2 \mathrm{D} 3,0,0,0,-\mathrm{D} 1 \mathrm{D} 3, \mathrm{D} 1^{2}, 0,-\mathrm{D} 1 \mathrm{D} 2,0,0 \\
\mathrm{D} 4^{2}, 0,0, \mathrm{D} 1^{2}, 0,0,0,0,0,-2 \mathrm{D} 1 \mathrm{D} 4 \\
\mathrm{D}^{2}, 0, \mathrm{D} 1^{2}, 0,0,0,0,-2 \mathrm{D} 1 \mathrm{D} 3,0,0 \\
\mathrm{D} 2^{2}, \mathrm{D} 1^{2}, 0,0,-2 \mathrm{D} 1 \mathrm{D} 2,0,0,0,0,0 \\
0, \mathrm{D} 3 \mathrm{D} 4,0,0,0,-\mathrm{D} 2 \mathrm{D} 4, \mathrm{D} 2^{2}, 0,-\mathrm{D} 2 \mathrm{D} 3,0 \\
0, \mathrm{D} 4^{2}, 0, \mathrm{D} 2^{2}, 0,0,0,0,-2 \mathrm{D} 2 \mathrm{D} 4,0 \\
0, \mathrm{D} 3^{2}, \mathrm{D} 2^{2}, 0,0,-2 \mathrm{D} 2 \mathrm{D} 3,0,0,0,0 \\
0,-\mathrm{D} 1 \mathrm{D} 4,0,0, \mathrm{D} 2 \mathrm{D} 4,0,0,0, \mathrm{D} 1 \mathrm{D} 2,-\mathrm{D} 2^{2} \\
0,-\mathrm{D} 1 \mathrm{D} 3,0,0, \mathrm{D} 2 \mathrm{D} 3, \mathrm{D} 1 \mathrm{D} 2,0,-\mathrm{D} 2^{2}, 0,0 \\
0,0, \mathrm{D} 4^{2}, \mathrm{D} 3^{2}, 0,0,-2 \mathrm{D} 3 \mathrm{D} 4,0,0,0 \\
0,0,-\mathrm{D} 2 \mathrm{D} 4,0,0, \mathrm{D} 3 \mathrm{D} 4, \mathrm{D} 2 \mathrm{D} 3,0,-\mathrm{D} 3^{2}, 0 \\
0,0,-\mathrm{D} 1 \mathrm{D} 4,0,0,0, \mathrm{D} 1 \mathrm{D} 3, \mathrm{D} 3 \mathrm{D} 4,0,-\mathrm{D} 3^{2} \\
0,0, \mathrm{D} 1 \mathrm{D} 2,0, \mathrm{D} 3^{2},-\mathrm{D} 1 \mathrm{D} 3,0,-\mathrm{D} 2 \mathrm{D} 3,0,0 \\
0,0,0, \mathrm{D} 2 \mathrm{D} 3,0, \mathrm{D} 4{ }^{2},-\mathrm{D} 2 \mathrm{D} 4,0,-\mathrm{D} 3 \mathrm{D} 4,0 \\
0,0,0, \mathrm{D} 1 \mathrm{D} 3,0,0,-\mathrm{D} 1 \mathrm{D} 4, \mathrm{D} 4^{2}, 0,-\mathrm{D} 3 \mathrm{D} 4 \\
0,0,0, \mathrm{D} 1 \mathrm{D} 2, \mathrm{D} 4^{2}, 0,0,0,-\mathrm{D} 1 \mathrm{D} 4,-\mathrm{D} 2 \mathrm{D} 4 \\
0,0,0,0, \mathrm{D} 3 \mathrm{D} 4,-\mathrm{D} 1 \mathrm{D} 4, \mathrm{D} 1 \mathrm{D} 2,0,0,-\mathrm{D} 2 \mathrm{D} 3 \\
0,0,0,0,0,-\mathrm{D} 1 \mathrm{D} 4,0, \mathrm{D} 2 \mathrm{D} 4, \mathrm{D} 1 \mathrm{D} 3,-\mathrm{D} 2 \mathrm{D} 3
\end{array}\right]
$$

The parametrization of the $D$-module $M / t(M)=D^{1 \times 10} / D^{1 \times 20} E x t 1[2]$ is defined by:

$>\operatorname{Ext1}[3]$

$$
\left[\begin{array}{cccc}
-2 \mathrm{D} 1 & 0 & 0 & 0 \\
0 & -2 \mathrm{D} 2 & 0 & 0 \\
0 & 0 & -2 \mathrm{D} 3 & 0 \\
0 & 0 & 0 & -2 \mathrm{D} 4 \\
-\mathrm{D} 2 & -\mathrm{D} 1 & 0 & 0 \\
0 & -\mathrm{D} 3 & -\mathrm{D} 2 & 0 \\
0 & 0 & -\mathrm{D} 4 & -\mathrm{D} 3 \\
-\mathrm{D} 3 & 0 & -\mathrm{D} 1 & 0 \\
0 & -\mathrm{D} 4 & 0 & -\mathrm{D} 2 \\
-\mathrm{D} 4 & 0 & 0 & -\mathrm{D} 1
\end{array}\right]
$$

Therefore, the underdetermined linear system of PDEs Ext1[2] $y=0$, which is associated with the $D$-module $M / t(M)=D^{1 \times 10} / D^{1 \times 20} \operatorname{Ext1}[2]$, is parametrized by $\operatorname{Ext1}[3]$, i.e., we have $y=\operatorname{Ext1}[3] z$, where $z=\left(z_{1}: z_{2}: z_{3}: z_{4}\right)^{T}$ are four potentials.

$>$ st:=time(): Ext2:=Exti(R_adj, Alg, 2): time()-st; Ext2[1]; 


$\left[\begin{array}{cccc}\mathrm{D} 1 & 0 & 0 & 0 \\ \mathrm{D} 4^{2} & 0 & 0 & 0 \\ \mathrm{D} 3 \mathrm{D} 4 & 0 & 0 & 0 \\ \mathrm{D} 2 \mathrm{D} 4 & 0 & 0 & 0 \\ \mathrm{D} 3^{2} & 0 & 0 & 0 \\ \mathrm{D} 2 \mathrm{D} 3 & 0 & 0 & 0 \\ \mathrm{D} 2^{2} & 0 & 0 & 0 \\ 0 & \mathrm{D} 2 & 0 & 0 \\ 0 & \mathrm{D} 4^{2} & 0 & 0 \\ 0 & \mathrm{D} 3 \mathrm{D} 4 & 0 & 0 \\ 0 & \mathrm{D} 1 \mathrm{D} 4 & 0 & 0 \\ 0 & \mathrm{D} 3^{2} & 0 & 0 \\ 0 & \mathrm{D} 1 \mathrm{D} 3 & 0 & 0 \\ 0 & \mathrm{D} 1^{2} & 0 & 0 \\ 0 & 0 & \mathrm{D} 3 & 0 \\ 0 & 0 & \mathrm{D} 4^{2} & 0 \\ 0 & 0 & \mathrm{D} 2 \mathrm{D} 4 & 0 \\ 0 & 0 & \mathrm{D} 1 \mathrm{D} 4 & 0 \\ 0 & 0 & \mathrm{D} 2^{2} & 0 \\ 0 & 0 & \mathrm{D} 1 \mathrm{D} 2 & 0 \\ 0 & 0 & \mathrm{D} 1^{2} & 0 \\ 0 & 0 & 0 & \mathrm{D} 4 \\ 0 & 0 & 0 & \mathrm{D} 3^{2} \\ 0 & 0 & 0 & \mathrm{D} 2 \mathrm{D} 3 \\ 0 & 0 & 0 & \mathrm{D} 1 \mathrm{D} 3 \\ 0 & 0 & 0 & \mathrm{D} 2^{2} \\ 0 & 0 & 0 & \mathrm{D} 1 \mathrm{D} 2 \\ 0 & 0 & 0 & \mathrm{D} 1^{2}\end{array}\right]$

We compute a free resolution of the linearized Ricci equations.

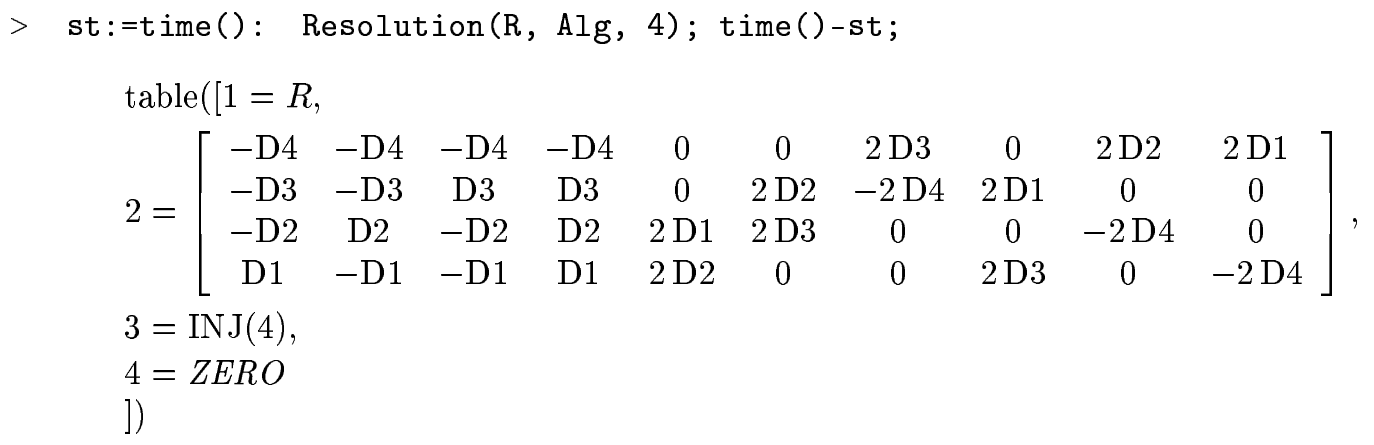

$$
7.540
$$

We compute a free resolution of the formal adjoint of the linearized Ricci equations.

$>$ st:=time(): Resolution(R_adj, Alg, 4); time()-st;

$$
\begin{aligned}
& \text { table }\left(\left[1=R_{-} a d j, 2=\left[\begin{array}{cccccccccc}
2 \mathrm{D} 1 & 0 & 0 & 0 & \mathrm{D} 2 & 0 & 0 & \mathrm{D} 3 & 0 & \mathrm{D} 4 \\
0 & 2 \mathrm{D} 2 & 0 & 0 & \mathrm{D} 1 & \mathrm{D} 3 & 0 & 0 & \mathrm{D} 4 & 0 \\
0 & 0 & 2 \mathrm{D} 3 & 0 & 0 & \mathrm{D} 2 & \mathrm{D} 4 & \mathrm{D} 1 & 0 & 0 \\
0 & 0 & 0 & 2 \mathrm{D} 4 & 0 & 0 & \mathrm{D} 3 & 0 & \mathrm{D} 2 & \mathrm{D} 1
\end{array}\right]\right.\right. \text {, } \\
& 3=\mathrm{INJ}(4) \text {, } \\
& 4=Z E R O \\
& \text { ]) }
\end{aligned}
$$




\section{A.7 Lie-Poisson structures}

Finally, let us notice that the results developed in this paper can be applied to underdetermined linear systems of PDEs with variable coefficients that appear in some problems of mathematical physics. For instance, let us give an example coming from the study of Lie-Poisson structures [3, 35].

$>A l g:=$ Define0reAlgebra $(\operatorname{dif} f=[D 1, x 1], \operatorname{diff}=[D 2, x 2], \operatorname{diff}=[D 3, x 3]$,

$>$ polynom $=\{\mathrm{x} 1, \mathrm{x} 2, \mathrm{x} 3\})$ :

The following example appears in the study of the $E_{2}$ algebra [3]. The authors of [3] investigated the possibility to parametrize all the solutions of the system of PDEs defined by the following matrix.

$>\quad \mathrm{R}:=\operatorname{evalm}([[\mathrm{x} 1 * \mathrm{D} 3, \mathrm{x} 2 * \mathrm{D} 3,0]$,
$>\quad[-\mathrm{x} 1 * \mathrm{D} 2+\mathrm{x} 2 * \mathrm{D} 1,-1, \mathrm{x} 2 * \mathrm{D} 3]$,
$>\quad[-1,-\mathrm{x} 2 * \mathrm{D} 1+\mathrm{x} 1 * \mathrm{D} 2, \mathrm{x} 1 * \mathrm{D} 3]]) ;$
$R:=\left[\begin{array}{ccc}x 1 \mathrm{D} 3 & x 2 \mathrm{D} 3 & 0 \\ -x 1 \mathrm{D} 2+x 2 \mathrm{D} 1 & -1 & x 2 \mathrm{D} 3 \\ -1 & -x 2 \mathrm{D} 1+x 1 \mathrm{D} 2 & x 1 \mathrm{D} 3\end{array}\right]$

Let $D=A_{3}$ be the Weyl algebra (see Example 2.3) and $M=D^{1 \times 3} / D^{1 \times 3} R$ be the left $D$-module associated with $R$. The previous problem can be solved by computing $\operatorname{ext}_{D}^{1}(N, D)$, where $\tilde{N}=$ $D^{1 \times 3} / D^{1 \times 3} \theta(R)$ is a left $D$-module and $\theta$ is the involution defined in 2 of Example 6.3.

$>\operatorname{Ext} 1:=\operatorname{Exti}$ (Involution(R, Alg), Alg, 1);

$$
\begin{aligned}
& \text { Ext1 }:=\left[\begin{array}{ccc}
\mathrm{D} 3 & 0 & 0 \\
-x 2 \mathrm{D} 1+x 1 \mathrm{D} 2 & 0 & 0 \\
0 & x 2 \mathrm{D} 3 & 0 \\
0 & x 1 \mathrm{D} 3 & 0 \\
0 & -x 2 \mathrm{D} 1+x 1 \mathrm{D} 2 & 0 \\
0 & 0 & 1
\end{array}\right],\left[\begin{array}{ccc}
x 1 & x 2 & 0 \\
\mathrm{D} 1 & \mathrm{D} 2 & \mathrm{D} 3 \\
-1 & -x 2 \mathrm{D} 1+x 1 \mathrm{D} 2 & x 1 \mathrm{D} 3
\end{array}\right], \\
& \left.\left[\begin{array}{c}
-x 2 \mathrm{D} 3 \\
x 1 \mathrm{D} 3 \\
-x 1 \mathrm{D} 2+x 2 \mathrm{D} 1
\end{array}\right]\right]
\end{aligned}
$$

We find that the system $R(F: G: H)^{T}=0$ is not parametrizable because there exist two torsion elements in the left $D$-module $M$

$$
\left\{\begin{array}{l}
\Phi_{1}=x_{1} F+x_{2} G \\
\Phi_{2}=\partial_{1} F+\partial_{2} G+\partial_{3} H
\end{array}\right.
$$

which satisfy:

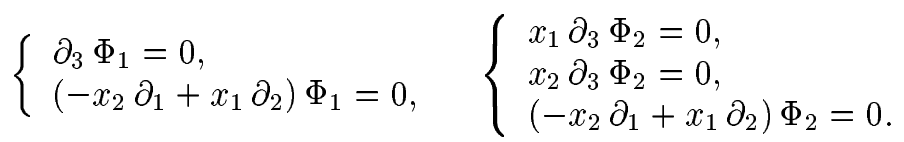

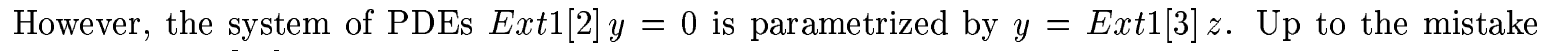
underlined in [35] concerning the existence of the torsion elements, we recover the parametrization exhibited in [3]. We refer the interested reader to [35] for more details.

The previous computation shows $M$ is not a torsion left $D$-module even if $R$ is a square matrix. Hence, $R$ does not have full row rank. This result can be checked by computing a free resolution of the left $D$-module $M=D^{1 \times 3} / D^{1 \times 3} R$.

$>$ Free:=FreeResolution (R,Alg);

$$
\begin{aligned}
& \text { Free }:=\operatorname{table}\left(\left[1=\left[\begin{array}{ccc}
x 1 \mathrm{D} 3 & x 2 \mathrm{D} 3 & 0 \\
-x 1 \mathrm{D} 2+x 2 \mathrm{D} 1 & -1 & x 2 \mathrm{D} 3 \\
-1 & -x 2 \mathrm{D} 1+x 1 \mathrm{D} 2 & x 1 \mathrm{D} 3
\end{array}\right],\right.\right. \\
& 2=\left[\begin{array}{lll}
-x 2 \mathrm{D} 1+x 1 \mathrm{D} 2 & x 1 \mathrm{D} 3 & -x 2 \mathrm{D} 3
\end{array}\right] \text {, } \\
& 3=\mathrm{INJ}(1) \\
& \text { ]) }
\end{aligned}
$$

$\mathrm{RR} \mathrm{n}^{\circ} 5181$ 
In particular, the left $D$-module $L=D^{1 \times 3} / D$ Free[2] is torsion-free as we have $L \cong D^{1 \times 3} R \subset D^{1 \times 3}$.

$$
\begin{aligned}
& >\text { ext: }=\operatorname{Exti}(\text { Involution(Free [2], Alg), Alg, 1); } \\
& \text { ext }:=\left[[1],\left[\begin{array}{lll}
-x 2 \mathrm{D} 1+x 1 \mathrm{D} 2 & x 1 \mathrm{D} 3 & -x 2 \mathrm{D} 3
\end{array}\right],\left[\begin{array}{ccc}
\mathrm{D} 3 & x 1 \mathrm{D} 3 & 0 \\
-\mathrm{D} 2 & -x 1 \mathrm{D} 2+x 2 \mathrm{D} 1 & x 2 \\
-\mathrm{D} 1 & -1 & x 1
\end{array}\right]\right]
\end{aligned}
$$

Therefore, the matrices $R \in D^{3 \times 3}$ and $\operatorname{ext}[3]$ are two parametrizations of the left $D$-module $L=$ $D^{1 \times 3} / D$ Free[2]. Let us compute $\operatorname{rank}_{D} L$.

$>\operatorname{OreRank}($ Free $[2], \mathrm{Alg})$;

2

Hence, we conclude that $y=R z$ and $y=\operatorname{ext}[3] z$ are not minimal parametrizations of the system $R(F: G: H)^{T}=0$ because they depend on three arbitrary functions of $\left(x_{1}, x_{2}, x_{3}\right)$. Let us compute some minimal parametrizations of the system $R(F: G: H)^{T}=0$.

$>\quad \mathrm{P}:=$ MinimalParametrizations (Free [2],Alg);

$$
P:=\left[\left[\begin{array}{cc}
\mathrm{D} 3 & x 1 \mathrm{D} 3 \\
-\mathrm{D} 2 & -x 1 \mathrm{D} 2+x \mathscr{D} 1 \\
-\mathrm{D} 1 & -1
\end{array}\right],\left[\begin{array}{cc}
\mathrm{D} 3 & 0 \\
-\mathrm{D} 2 & x 2 \\
-\mathrm{D} 1 & x 1
\end{array}\right],\left[\begin{array}{cc}
x 1 \mathrm{D} 3 & 0 \\
-x 1 \mathrm{D} 2+x 2 \mathrm{D} 1 & x \mathscr{2} \\
-1 & x 1
\end{array}\right]\right]
$$

Then, we can check that $y=P[1] z, y=P[2] z$ and $y=P[3] z$ are three minimal parametrizations of the system $R(F: G: H)^{T}=0$, where $z=\left(z_{1}: z_{2}\right)^{T}$, and $z_{1}$ and $z_{2}$ are two arbitrary functions of $x_{1}, x_{2}$ and $x_{3}$.

$>\operatorname{seq}($ SyzygyModule(P $[\mathrm{i}], \mathrm{Alg}), \mathrm{i}=1 . .3)$;

$$
\begin{aligned}
& \% 1, \% 1, \% 1 \\
& \% 1:=[-x 2 \mathrm{D} 1+x 1 \mathrm{D} 2 \quad x 1 \mathrm{D} 3 \quad-x 2 \mathrm{D} 3]
\end{aligned}
$$

To finish, let us consider the Ore algebra $B_{3}=\mathbb{R}\left(x_{1}, x_{2}, x_{3}\right)\left[\partial_{1} ; \sigma_{1}, \delta_{1}\right]\left[\partial_{2} ; \sigma_{2}, \delta_{2}\right]\left[\partial_{3} ; \sigma_{3}, \delta_{3}\right]$, where $\sigma_{i}$ and $\delta_{i}$ are defined as in the last part of Example 2.3 and let us define the left $B_{3}$-module $M^{\prime}=$ $B_{3}^{1 \times 3} / B_{3}^{1 \times 3} R$ associated with $R$ over $B_{3}$. Since the domain of coefficients of $B_{3}$ is the quotient field of $\mathbb{R}\left[x_{1}, x_{2}, x_{3}\right]$, we are going to use the Weyl algebra $D=A_{3}$ (see Example 2.3) and allow our algorithms to divide by non-zero polynomials in $x_{1}, x_{2}, x_{3}$. This is taken into account by ExtiRat below.

$>$ st:=time(): $\operatorname{ExtiRat(Involution(R,Alg),Alg,~1);~}$

$>$ time()-st;

$$
\begin{aligned}
& {\left[\left[\begin{array}{cc}
\mathrm{D} 3 & 0 \\
-x 1 \mathrm{D} 2+x 2 \mathrm{D} 1 & 0 \\
0 & \mathrm{D} 3 \\
0 & -x 1 \mathrm{D} 2+x 2 \mathrm{D} 1
\end{array}\right],\left[\begin{array}{ccc}
x 1 & x 2 & 0 \\
0 & -x 1^{2} \mathrm{D} 2+x 1 x 2 \mathrm{D} 1-x 2 & -x 1^{2} \mathrm{D} 3
\end{array}\right]\right. \text {, }} \\
& \left.\left[\begin{array}{c}
x 2 \mathrm{D} 3 \\
-x 1 \mathrm{D} 3 \\
-x 2 \mathrm{D} 1+x 1 \mathrm{D} 2
\end{array}\right]\right]
\end{aligned}
$$

0.670

We find that the system $R(F: G: H)^{T}=0$ is not parametrizable because there exist two torsion elements

$$
\left\{\begin{array}{l}
\Phi_{1}=x_{1} F+x_{2} G \\
\Phi_{2}=\left(-x_{1}^{2} \partial_{2}+x_{1} x_{2} \partial_{1}-x_{2}\right) G-x_{1}^{3} \partial_{3} H
\end{array}\right.
$$


which both satisfy the system:

$$
\left\{\begin{array}{l}
\partial_{3} \Phi_{i}=0 \\
\left(-x_{1} \partial_{2}+x_{2} \partial_{1}\right) \Phi_{i}=0, \quad i=1,2 .
\end{array}\right.
$$

We recover the torsion element $\Phi_{1}$ exhibited in [35] as well as the parametrization of the torsion-free left $B_{3}$-module $M^{\prime} / t\left(M^{\prime}\right)$ given in [3].

\section{References}

[1] Adams, W. W. and Loustaunau, P. An Introduction to Gröbner Bases, American Mathematical Society, 1994.

[2] Becker, T. and Weispfenning, V. Gröbner Bases. A Computational Approach to Commutative Algebra, Springer, 1993.

[3] Bender, C. M., Dunne, G. V. and Mead, L. R. "Underdetermined systems of partial differential equations", Journal of Mathematical Physics, vol. 41 (2000), 6388-6398.

[4] N. Bourbaki, Commutative Algebra Chap. 10, Springer Verlag.

[5] Chyzak, F. Fonctions holonomes en calcul formel, PhD thesis, Ecole Polytechnique, 27/05/1998.

[6] Chyzak, F. "Mgfun Project", http://algo.inria.fr/chyzak/mgfun.html.

[7] Chyzak, F. and Salvy, B. "Non-commutative elimination in Ore algebras proves multivariate identities", J. Symbolic Computation, vol. 26 (1998), 187-227.

[8] Chyzak, F., Quadrat, A. and Robertz, D. "Linear control systems over Ore algebras: Effective algorithms for the computation of parametrizations", CDRom of the Workshop on Time-Delay Systems (TDS03), IFAC Workshop, INRIA Rocquencourt (France) (08-10/09/03).

[9] Chyzak, F., Quadrat, A. and Robertz, D. "OreModules: A symbolic package for the study of multidimensional linear systems", to appear in the proceedings of MTNS04, Leuven (Belgium) (05-09/07/04).

[10] Cotroneo, T. Algorithms in Behavioral Systems Theory, $\mathrm{PhD}$ thesis, University of Groningen (The Netherlands), 18/05/2001.

[11] Fliess, M. and Mounier, H. "Controllability and observability of linear delay systems: an algebraic approach", ESAIM COCV, vol. 3 (1998), pp. 301-314.

[12] Kredel, H. Solvable Polynomial Rings, Verlag Shaker, Aachen, 1993.

[13] Gluesing-Luerssen, H., Rosenthal, J. and Weiner, P. A. "Duality between multidimensional convolutional codes and systems" in F. Colonius, U. Helmke, F. Wirth, and D. Praetzel-Wolters, editors, Advances in Mathematical Systems Theory, A Volume in Honor of Diederich Hinrichsen, 135-150. Birkhäuser, 2000.

[14] La Scala, R. and Stillman, M. "Strategies for computing minimal free resolutions", J. Symbolic Computation, vol. 26 (1998), pp 409-431.

[15] Landau, L. and Lifchitz, E. "Physique théorique. Tome 2: Théorie des champs", MIR, 1989.

[16] Le Vey, G. "Some remarks on solvability and various indices for implicit differential equations", Numerical Algorithms, vol. 19 (1998), pp. 127-145.

[17] McConnell, J. C. and Robson, J. C. Noncommutative Noetherian Rings, American Mathematical Society, 2000.

[18] Oberst, U. "Multidimensional constant linear systems", Acta Appl. Math., vol. 20 (1990), pp. $1-175$.

[19] Manitius, A. "Feedback controllers for a wind tunnel model involving a delay: analytical design and numerical simulations", IEEE Trans. Autom. Contr. vol. 29 (1984), pp. 1058-1068. 
[20] Mounier, H., Propriétés structurelles des systèmes linéaires à retards: aspects théoriques et pratiques, PhD Thesis, University of Orsay, France, 1995.

[21] Mounier, H., Rudolph, J., Fliess, M. and Rouchon, P. "Tracking control of a vibrating string with an interior mass viewed as delay system", ESAIM COCV, vol. 3 (1998), pp. 315-321.

[22] Pillai, H. K. and Shankar, S. "A behavioral approach to control of distributed systems", SIAM J. Control \& Optimization 37 (1998), 388-408.

[23] Polderman, J. W. and Willems, J. C. Introduction to Mathematical Systems Theory. A Behavioral Approach, TAM 26, Springer, 1998.

[24] Pommaret, J.-F., "Dualité différentielle et applications", C.R. Acad. Sci. Paris, Série I 320 (1995), pp. 1225-1230.

[25] Pommaret, J.-F., Partial Differential Control Theory, Kluwer, 2001.

[26] Pommaret, J.-F. and Quadrat, A. "Localization and parametrization of linear multidimensional control systems", Systems \& Control Letters, vol. 37 (1999), pp. 247-260.

[27] Pommaret, J.-F. and Quadrat, A. "Algebraic analysis of linear multidimensional control systems", IMA J. Control and Optimization, vol. 16 (1999), pp. 275-297.

[28] Pommaret, J.-F. and Quadrat, A. "Equivalences of linear control systems", Proceedings of MTNS00, Perpignan (France), available at http://www-sop.inria.fr/cafe/Alban.Quadrat/index.html.

[29] Pommaret, J.-F. and Quadrat, A. "A functorial approach to the behaviour of multidimensional control system", Applied Mathematics and Computer Science, vol. 13 (2003), pp. 7-13.

[30] Quadrat, A. Analyse algébrique des systèmes de contrôle linéaires multidimensionnels, $\mathrm{PhD}$ thesis, Ecole Nationale des Ponts et Chaussées (France) 23/09/1999.

[31] Quadrat, A. "Extended Bézout identities", Proceedings of ECC01, Porto (Portugal), available at http://www-sop.inria.fr/cafe/Alban.Quadrat/index.html.

[32] Rocha, P. Structure and Representation of 2-D systems, $\mathrm{PhD}$ thesis, University of Groningen (The Netherlands), 1990.

[33] Rotman, J. J. An Introduction to Homological Algebra, Academic Press, 1979.

[34] Salamon, D. Control and Observation of Neutral Systems, Pitman, 1984.

[35] Seiler, W. M. "Involution analysis of the partial differential equations characterising Hamiltonian vector fields", Journal of Mathematical Physics, vol. 44 (2003), pp. 1173-1182.

[36] Wood, J. "Modules and behaviours in $n$ D systems theory", Multidimensional Dimensional Systems and Signal Processing, vol. 11 (2000), pp. 11-48.

[37] Zerz, E. Topics in Multidimensional Linear Systems Theory, Lecture Notes in Control and Information Sciences 256, Springer, London (2000). 


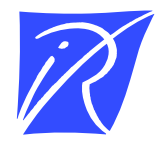

\section{Unité de recherche INRIA Sophia Antipolis 2004, route des Lucioles - BP 93 - 06902 Sophia Antipolis Cedex (France)}

Unité de recherche INRIA Futurs : Parc Club Orsay Université - ZAC des Vignes 4, rue Jacques Monod - 91893 ORSAY Cedex (France)

Unité de recherche INRIA Lorraine : LORIA, Technopôle de Nancy-Brabois - Campus scientifique 615, rue du Jardin Botanique - BP 101 - 54602 Villers-lès-Nancy Cedex (France)

Unité de recherche INRIA Rennes : IRISA, Campus universitaire de Beaulieu - 35042 Rennes Cedex (France)

Unité de recherche INRIA Rhône-Alpes : 655, avenue de l'Europe - 38334 Montbonnot Saint-Ismier (France)

Unité de recherche INRIA Rocquencourt : Domaine de Voluceau - Rocquencourt - BP 105 - 78153 Le Chesnay Cedex (France)

INRIA - Domaine de Voluceau - Rocquencourt, BP 105 - 78153 Le Chesnay Cedex (France)

http://www.inria.fr

ISSN 0249-6399 